February, 2007

\title{
Light-Cone Distribution Amplitudes of Axial-vector Mesons
}

\author{
KWEI-ChOU YANG \\ Department of Physics, Chung Yuan Christian University \\ Chung-Li, Taiwan 320, Republic of China
}

\begin{abstract}
We have presented a detailed study of twist-2 and twist-3 light-cone distribution amplitudes of $1^{3} P_{1}$ and $1^{1} P_{1}$ axial-vector mesons, based on QCD conformal partial wave expansion. Applying equations of motion, the twist-three two-parton light-cone distribution amplitudes can be expressed in terms of leading-twist and twist-three three-parton light-cone distribution amplitudes. The relevant G-parity invariant and violating parameters, containing the corrections due to the $\mathrm{SU}(3)$ breaking effects, are evaluated from the QCD sum rule method. The results for axial-vector decay constants of $1^{3} P_{1}$ states are presented. The values of tensor decay constants and Gegenbauer moments of the leading twist distribution amplitudes for $1^{1} P_{1}$ states are updated. Using Gell-Mann-Okubo mass formula, the mixing angle for the $f_{8}$ and $f_{1}$ of $1^{3} P_{1}$ states is $\theta_{3} P_{1} \sim 38^{\circ}$, and that for $h_{8}$ and $h_{1}$ of $1^{1} P_{1}$ states is $\theta_{1} P_{1} \sim 10^{\circ}$. The detailed properties for physical states $f_{1}(1285), f_{1}(1420), h_{1}(1170)$, and $h_{1}(1380)$ are given. Assuming the mixing angle between $K_{1 A}$ and $K_{1 B}$ to be $\theta_{K}=45^{\circ}$ or $-45^{\circ}$, we also give the detailed study for $K_{1}(1270)$ and $K_{1}(1400)$. Using the conformal partial wave expansion, we obtain the models for light-cone distribution amplitudes, containing contributions up to conformal spin $9 / 2$. It is interesting to note that some distribution amplitudes have significant asymmetric behaviors, which should be phenomenologically attractive.
\end{abstract}




\section{Introduction}

For an energetic light hadron moving nearly on the light-cone in the reference frame, its distribution amplitudes can be described by a set of light-cone distribution amplitudes (LCDAs) [1, 2]. The LCDAs are governed by the special collinear subgroup $S L(2, \mathbb{R})$ of the conformal group[1, 2]. There are four generators for the $S L(2, \mathbb{R})$ group. Three of generators describe the generalized rotations in the $S L(2, \mathbb{R})$ space, where the rotational invariance is characterized by the so-called conformal spin $j$, in analogy to the orbital quantum number in quantum mechanics of having spherically symmetric potential. The remaining generator counting the collinear twist commutes with the rest generators in the $S L(2, \mathbb{R})$ group; thus, in the mathematical spirit, the concept of "collinear twist" is equivalent to the "energy" in quantum mechanics, and, in other words, the $S L(2, \mathbb{R})$ group are satisfied by the same Lie algebra as the $O(3)$ group.

The conformal partial wave expansion of a light-cone distribution amplitude is fully analogous to the partial wave expansion of a wave function in quantum mechanics. Each conformal partial wave is labeled by the specific conformal spin $j$. On the other hand, for a hadron moving nearly on the light-cone, the transverse separation of the partons corresponding to the two-dimensional transverse plane $\left(0, x_{1}, x_{2}, 0\right)$ is characterized by the $S L(2, \mathbb{C})$ group. Moreover, the collinear $S L(2, \mathbb{R})$ and transverse $S L(2, \mathbb{C})$ groups are not independent because they share the same dilation generator. Integrating out the transverse degrees of freedom, which is governed by renormalization group $(R G)$ equation, yields the scale-dependent behavior for each conformal partial wave amplitude of a hadronic LCDA. The conformal invariance in QCD exhibits that for leading twist LCDAs there is no mixing among conformal partial wave amplitudes with different conformal spins to leading logarithmic accuracy, while, for higher twist LCDAs, because there may exist multiple operators with the same conformal spin, the corresponding conformal partial amplitudes with the same conformal spin can mix as the scale is changed. Roughly speaking, to leading logarithmic accuracy, the anomalous dimensions rise logarithmically with conformal spin. This implies that contributions of conformal operators with high conformal spins are suppressed at large scale; for instance, the well-known leading twist LCDA of the pion reads $\phi_{\pi}(u, \mu \rightarrow \infty) \rightarrow 6 u(1-u)$, i.e., the asymptotic amplitude with the lowest conformal spin 2 , where $u$ is the momentum fraction carried by the quark (or anti-quark) in the pion. It is interesting to note that in the BFKL approach [3, 4, 5, 6], the QCD scattering amplitude in the large energy limit is related to the $S L(2, \mathbb{C}$ ) group (see, e.g., the discussion in Ref. [2]).

Understanding the hadronic LCDAs is very important since in the QCD description of various exclusive processes the amplitudes can be represented as the hadronic LCDAs convoluted with the interacting hard kernel. The studies for the hadronic LCDAs were first done by Chernyak and Zhinitsky [7]. They used the QCD sum rule approach to calculate the moments of LCDAs of $\pi, K, \rho, K^{*}$ and some baryons. Ten years later, a conformal description for multi-parton LCDAs was presented in more details in Ref. [1]. Most recently, using conformal expansion together with equations of motion (EOMs), the different twist 
LCDAs for the light pseudoscalar and vector mesons have been systematically studied in Refs. [8, 9, 10].

In the present paper, we devote to examining twist-2 and twist-3 LCDAs of light axialvector mesons with quantum numbers $1^{3} P_{1}, 1^{1} P_{1}$ and their mixtures. The motivation of this study is as follows. LCDAs of axial-vector mesons should be important for (exclusive) phenomenologies involving axial-vector mesons. $B^{0} \rightarrow K_{1}(1270) \gamma$ has recently been measured by Belle [11], whereas the real physical states $K_{1}(1270)$ and $K_{1}(1400)$ are the mixtures of ideal $1^{3} P_{1}\left(K_{1 A}\right)$ and $1^{1} P_{1}\left(K_{1 B}\right)$ states. The first charmless hadronic $B$ decay involving a $1^{3} P_{1}$ meson that has been observed is $B^{0} \rightarrow a_{1}^{ \pm}(1260) \pi^{\mp}$ [12, 13], which is relevant to measurements of the unitarity triangle $\alpha \equiv \phi_{2}$ of the Cabibbo-Kobayashi-Maskawa (CKM). BaBar has recently extracted the effective value $\alpha_{\text {eff }}$ from the measurements of $C P$ violating asymmetries in the decay $B^{0} \rightarrow a_{1}^{ \pm}(1260) \pi^{\mp}[14]$, where the bound on the difference $\Delta \alpha=\alpha-\alpha_{\text {eff }}$ can be constrained by using the broken $\mathrm{SU}(3)$ flavor symmetry [15]. On the other hand, $\mathcal{B}(B \rightarrow V A, A A)$ can be used to test QCD annihilation topology in $B$ decays and probe the new-physics signals, where $V \equiv$ vector meson and $A \equiv$ axial-vector meson [16, 17].

So far, there is no literature for the calculations of LCDAs of axial-vector mesons except my previous work about leading-twist LCDAs of $1^{1} P_{1}$ states [18]. In Ref. [18], we have calculated the Gegenbauer moments (with conformal spins 0, 1, and 2) of the leading twist LCDAs for the $b_{1}(1235), h_{1}(1380)$ and $K_{1 B}$ as well as their tensor decay constants. In this paper, we will derive all parameters, relevant to the two- and three-parton LCDAs of twist2 and -3 , with conformal spin up to $9 / 2$. We take into account $\mathrm{SU}(3)$ breaking effects in the sum rule calculations for G-parity invariant and violating parameters, where the latter parameters should vanish in the $\mathrm{SU}(3)$ limit. The decay constants for axial-vector mesons can be obtained by using the sum rules computed in Ref. [19], where the authors focused on the studies of the masses and used a small value of $\alpha_{s}$. Considering the current value of $\alpha_{s}$ and the renormalization group improvement for sum rules, we also examine the masses of the complete $1^{3} P_{1}$ set, which may offer information for the quality of the sum rules. Our results for parameters relevant to LCDAs of axial-vector mesons are essentially original. To determine the relative signs for parameters, one of the interpolating currents in the twopoint correlation function is adopted to be the local axial-vector current (or the local tensor current) for $1^{3} P_{1}$ states (or $1^{1} P_{1}$ states). However, because the resultant $f_{3,{ }^{3} P_{1}}^{\perp}$ sum rule does not show reliable quality, we thus resort to the diagonal correlation function to calculate the sum rule for this parameter. Fortunately, the related calculations can be found in Ref. [20], where the sum rule is instead used to analyze the couplings for pseudoscalar mesons. The detailed results are given in Subsec. 5.1.3. Except the formula $f_{3,{ }^{3} P_{1}}^{\perp}$ can be adopted directly from Ref. [20, we do not find any explicit results can be applied directly, but only two equations can be compared with the results in the massless quark limit in the literature. See discussions before and after Eq. (121) and after Eq. (244).

We also update the numerical results for the decay constants and leading twist LCDAs of the $1^{3} P_{1}$ states, due to the following reasons. First, here we consider the uncertainties of the 
condensates and strange quark mass, which were not fully included in Ref. [18]. Meanwhile, we re-examine the stability of the sum rules with requirement that the contributions from excited states and from the highest dimension term in OPE expansion can be well under control within the working Borel window. Second, in Ref. [18] we assumed that the property of $h_{1}(1170)$ is the same as that of $b_{1}(1235)$, and the quark content of $h_{1}(1370)$ is $\bar{s} s$. Instead, we study the pure $1^{1} P_{1}$ states here. We estimate the singlet-octet mixing angle by means of Gell-Mann-Okubo mass formula (see the detailed discussions in subsection 4.1). Consequently, the physical properties for the real states $h_{1}(1170)$ and $h_{1}(1380)$ are obtained. Third, the G-parity violating Gegenbauer moments were not evaluated correctly in Ref. [18] because the corrections arising from the $1^{3} P_{1}$ states had been ignored. In the sum rule calculations, for G-parity violating parameters, the contributions originating from $1^{1} P_{1}$ and $1^{3} P_{1}$ are always of the same order.

In the quark model, a $1^{3} P_{1}\left(1^{1} P_{1}\right)$ meson is represented as a constituent quark-antiquark pair with total spin $S=1(0)$ and angular momentum $L=1$ (1). Nevertheless, a real hadron in QCD language should be described in terms of a set of Fock states for which each state has the same quantum number as the hadron, and the leading-twist LCDAs are thus interpreted as amplitudes of finding the meson in states with a minimum number of partons. Interestingly, due to the G-parity, the leading-twist LCDA $\Phi_{\perp}\left(\Phi_{\|}\right)$of a $1^{3} P_{1}\left(1^{1} P_{1}\right)$ meson defined by the nonlocal tensor current (nonlocal axial-vector current) is antisymmetric under the exchange of quark and anti-quark momentum fractions in the $\mathrm{SU}(3)$ limit, whereas the $\Phi_{\|}\left(\Phi_{\perp}\right)$ defined by the nonlocal axial-vector current (nonlocal tensor current) is symmetric. The large magnitude of the first Gegenbauer moment of $\Phi_{\|}\left(\Phi_{\perp}\right)$ could have a large impact on the longitudinal fraction of factorization-suppressed $B$ decays involving a $1^{1} P_{1}$ (or $1^{3} P_{1}$ ) meson evaluated in the QCD factorization framework [16, 17]. Furthermore, $\Phi_{\perp}$ is relevant not only for exploring the tensor-type new-physics effects in $B$ decays [16] but also for $B \rightarrow K_{1} \gamma$ studies [21].

This paper is organized as follows. A brief introduction for conformal partial wave expansions of LCDAs is provided in Sec. 2, where we first introduce the $\operatorname{SL}(2, \mathbb{R})$ group, and then discuss the asymptotic LCDAs, two-parton and three-parton distribution amplitudes. In earlier days, Appell's polynomials were used to form the conformal basis in expanding three-parton distribution amplitudes. However, the Appell's polynomials are not mutual orthogonal (see, e.g., Refs. [2, 22]). Following Ref. [2, 22, 23], we thus use the orthogonal basis, which will be introduced in Sec. 2.2. to expand the three-parton LCDAs of twist-3. The detailed collinear twist properties of LCDAs are collected in Appendix A.

Sec. 3 presents the notations, that we use in the paper, and definitions of LCDAs of axial-vector mesons. Moreover, we apply the EOMs to obtain the relations among the twist-two and twist-three light-cone distribution amplitudes, so that we can use a minimal number of independent nonperturbative parameters to describe the distribution amplitudes. EOMs in the QCD perturbative theory respect all symmetries given in the classical level; in other words, conformal operators related by EOMs should have the same conformal spin. Appendix B contains some relevant formulas of EOMs for the present work. 
Using the QCD sum rule technique [24], Secs. 4]and 5] are devoted to the determination of relevant parameters of leading-twist and twist-3 three-parton LCDAs, respectively. In Sec. 4, the physical properties of the axial-vector mesons such as the quark contents and decay constants are discussed. The relevant inputs in the calculation are collected in Appendix C. Sec. 6] contains explicit models for the twist-2 and twist-3 LCDAs of the axial-vector mesons. Finally, we summarize in Sec. 7 .

\section{Conformal Partial Wave Expansions of LCDAs}

The properties of fields living on the light-cone satisfy the $S L(2, \mathbb{R})$ group, which is a collinear subgroup of the full conformal group. The $S L(2, \mathbb{R})$ group is governed by four generators $\mathbf{P}_{+}, \mathbf{M}_{-+}, \mathbf{D}$ and $\mathbf{K}_{-}$. Here and below we introduce two light-like vectors $n^{\mu}$ and $\bar{n}^{\mu}$ which satisfy $n_{\mu} n^{\mu}=\bar{n}_{\mu} \bar{n}^{\mu}=0$ and $n_{\mu} \bar{n}^{\mu}=1$, so that for a general vector $A$ we define $A_{-} \equiv A_{\mu} \bar{n}^{\mu}$ and $A_{+} \equiv A_{\mu} n^{\mu}$. In analogy to the space rotation in quantum mechanics, the three linear combinations of the $S L(2, \mathbb{R})$ generators can describe hyperbolic rotations, and have the following commutation relations:

$$
\left[\mathbf{L}_{0}, \mathbf{L}_{\mp}\right]=\mp \mathbf{L}_{\mp}, \quad\left[\mathbf{L}_{-}, \mathbf{L}_{+}\right]=-2 \mathbf{L}_{0}
$$

where

$$
\mathbf{L}_{+}=-i \mathbf{P}_{\mu} n^{\mu}, \quad \mathbf{L}_{-}=(i / 2) \mathbf{K}_{\mu} \bar{n}^{\mu}, \quad \mathbf{L}_{0}=-(i / 2)\left(\mathbf{D}-\mathbf{M}_{\mu \nu} \bar{n}^{\mu} n^{\nu}\right),
$$

with $\mathbf{P}_{\mu}, \mathbf{K}_{\mu}, \mathbf{D}$ and $\mathbf{M}_{\mu \nu}$ being the translation, special conformal transformation, dilation, and Lorentz generators, respectively [25]. Following the standard quantum mechanical technique, the above generators acting on a field $\Phi\left(x_{-}\right)$, which lives along the light-cone $\bar{n}^{\mu}$ (in other words, the state depends only on $\left.x^{\mu}=x_{-} n^{\mu}\right)$, yield

$$
\begin{aligned}
& {\left[\mathbf{L}_{+}, \Phi\left(x_{-}\right)\right]=-\partial_{+} \Phi\left(x_{-}\right) \equiv L_{+} \Phi\left(x_{-}\right)} \\
& {\left[\mathbf{L}_{-}, \Phi\left(x_{-}\right)\right]=\left(x_{-}^{2} \partial_{+}+2 j x_{-}\right) \Phi\left(x_{-}\right) \equiv L_{-} \Phi\left(x_{-}\right),} \\
& {\left[\mathbf{L}_{0}, \Phi\left(x_{-}\right)\right]=-\left(x_{-} \partial_{+}+j\right) \Phi\left(x_{-}\right) \equiv L_{0} \Phi\left(x_{-}\right),}
\end{aligned}
$$

where $j=(\ell+s) / 2$ with $\ell$ and $s$ being the field's scaling dimension and spin projection on the light-cone, respectively. $j$ is called the conformal spin since

$$
\left[\mathbf{L}^{2}, \Phi\left(x_{-}\right)\right]=j(j-1) \Phi\left(x_{-}\right) \equiv L^{2} \Phi\left(x_{-}\right)
$$

where

$$
\mathbf{L}^{2}=\mathbf{L}_{0}^{2}+\mathbf{L}_{1}^{2}+\mathbf{L}_{2}^{2}=\mathbf{L}_{0}^{2}-\mathbf{L}_{0}+\mathbf{L}_{+} \mathbf{L}_{-} .
$$

The remaining generator of the $S L(2, \mathbb{R})$ is symbolized as $\mathbf{E}=i\left(\mathbf{D}+\mathbf{M}_{-+}\right)$and satisfies

$$
\begin{aligned}
{\left[\mathbf{E}, \Phi\left(x_{-}\right)\right] } & =(\ell-s) \Phi\left(x_{-}\right), \\
{\left[\mathbf{E}, \mathbf{L}^{2}\right] } & =0, \quad\left[\mathbf{E}, \mathbf{L}_{0}\right]=0,
\end{aligned}
$$


where $\ell-s=t$ is called the collinear twist of the field $\Phi\left(x_{-}\right)$. See Appendix $\mathrm{A}$ for further discussions. Therefore the role of $\mathbf{E}$ is analogous to the Hamiltonian in quantum mechanics, and the twist corresponds to the eigenenergy of the Hamiltonian. In short, a LCDA defined by (non-local composite) field operators of a given collinear twist can be expanded by using the basis, which is made of the eigenstates of $L^{2}$ and $L_{0}$, as one does the partial wave expansion in quantum mechanics. Such expansions for LCDAs are so-called conformal partial wave expansions. In QCD, in the large scale limit and to leading logarithmic accuracy, we have $\ell=\ell^{\text {can }}+\gamma$, where $\ell^{\text {can }}$ and $\gamma$ are the canonical and anomalous dimensions of the field, respectively.

A generic multi-parton distribution amplitude is defined through the matrix element of a multi-local operator

$$
\left\langle 0\left|\Phi_{m}\left(x_{m-}\right) \cdots \Phi_{1}\left(x_{1-}\right)\right| p\right\rangle \sim \int[d u] \mathrm{e}^{-i p\left(x_{1} u_{1}+\cdots+x_{m} u_{m}\right)} \phi\left(u_{1}, \cdots, u_{m}\right),
$$

where $\int[d u]=\int_{0}^{1} d u_{1} \cdots \int_{0}^{1} d u_{m} \delta\left(\sum u_{i}-1\right), u_{i}=p_{i+} / p_{+}$are momentum fractions carried by partons " $i$ ", and the quantum fields $\Phi_{i}\left(x_{i-}\right)$ live on the light-cone. $\phi\left(u_{i}\right)$ can be expanded in the Hilbert space by using the basis defined by $L^{2}$ and $L_{0}$. In the expansion, the term with the lowest conformal spin and non-zero coefficient is the so-called the asymptotic distribution amplitude, which is usually dominant at the large scale for the general case [1, 2],

$$
\phi_{\mathrm{as}}\left(u_{i}\right)=\frac{\Gamma\left(2 j_{1}+\cdots+2 j_{m}\right)}{\Gamma\left(2 j_{1}\right) \cdots \Gamma\left(2 j_{m}\right)} u_{1}^{2 j_{1}-1} u_{2}^{2 j_{2}-1} \cdots u_{m}^{2 j_{m}-1},
$$

where the normalization is chosen such that $\int\left[d u_{i}\right] \phi_{\text {as }}\left(u_{i}\right)=1$.

\subsection{Two-parton distribution amplitudes}

The study for conformal expansion of two-parton distribution amplitudes has been given by Ohrndorf [26] (see, e.g., the discussion in Ref. 2]). Consider

$$
\left\langle 0\left|\Phi_{2}^{j_{2}}\left(x_{2}\right) \Phi_{1}^{j_{1}}\left(x_{1}\right)\right| p\right\rangle=\int[d u] \mathrm{e}^{-i p\left(x_{1} u_{1}+x_{2} u_{2}\right)} \phi\left(u_{1}, u_{2} ; \mu\right)
$$

Using the conformal basis, the two-parton distribution amplitude with a given collinear twist can be written as

$$
\phi\left(u_{1}, u_{2} ; \mu\right)=\phi_{a s} \sum_{j-j_{1}-j_{2}=0}^{\infty} \phi_{j-j_{1}-j_{2}}(\mu) \mathbb{P}_{j-j_{1}-j_{2}}^{j_{1}, j_{2}}\left(u_{1}, u_{2}\right) .
$$

where

$$
\mathbb{P}_{N}^{j_{1}, j_{2}}\left(u_{1}, u_{2}\right)=\sum_{n_{1}+n_{2}=N} \frac{u_{1}^{n_{1}}\left(-u_{2}\right)^{n_{2}}\left(\begin{array}{c}
N \\
n_{2}
\end{array}\right)}{\Gamma\left(n_{1}+2 j_{1}\right) \Gamma\left(n_{2}+2 j_{2}\right)}=\left(u_{1}+u_{2}\right)^{N} P_{N}^{2 j_{1}-1,2 j_{2}-1}\left(\frac{u_{1}-u_{2}}{u_{1}+u_{2}}\right)
$$

\footnotetext{
${ }^{1}$ For simplicity, it will be just denoted as "twist" in this paper. Conventionally, we call $\ell^{\text {can }}-s$ as the twist, instead of using $\ell-s$, where $\ell^{\text {can }}$ is the canonical dimension.
} 
with $P_{N}^{a, b}(x)$ being the so-called Jacobi polynomials. Here the conformal spins of $\Phi_{1}^{j_{1}}$ and $\Phi_{2}^{j_{2}}$ are $j_{1}$ and $j_{2}$, respectively.

As for the case of the twist-2 (leading-twist) distribution amplitude of an axial-vector meson, we have the conformal spins $j_{1}=j_{2}=1$ for both quark and anti-quark fields. The leading-twist LCDA thus reads

$$
\phi\left(u_{1}, u_{2} ; \mu\right)=6 u_{1} u_{2} \sum_{l=0}^{\infty} a_{l}(\mu) C_{l}^{3 / 2}\left(u_{1}-u_{2}\right)
$$

where $P_{j-2}^{1,1}\left(u_{1}-u_{2}\right) \sim C_{l}^{3 / 2}\left(u_{1}-u_{2}\right)$ with $j-2=l$.

\subsection{Three-parton distribution amplitudes}

We follow the method suggested by Braun et al. [22], who first applied it to baryon distribution amplitudes, to construct the three-parton distribution amplitudes of an axial-vector meson. The traditional choice is to expand three-parton distribution amplitudes in terms of Appell's polynomials. However, this basis is inconvenient for calculations since Appell's polynomials are not mutually orthogonal. An orthonormal "conformal basis" of functions $J_{N n}^{(12) 3}\left(u_{i}\right)=(-1)^{N} Y_{J j}^{(12) 3}\left(u_{i}\right) / 2$ can be defined by requiring that the total three-parton conformal spin $J=j_{1}+j_{2}+j_{3}+N$ of an eigenstate is fixed, and, moreover, the definite value of the conformal spin of the two-parton channel (12) is given as $j=j_{1}+j_{2}+n$ with $n=0, \cdots, N$. The results of $Y_{J j}^{(12) 3}$ can be written as

$$
Y_{J j}^{(12) 3}\left(u_{i}\right)=\left(1-u_{3}\right)^{j-j_{1}-j_{2}} P_{J-j-j_{3}}^{\left(2 j_{3}-1,2 j-1\right)}\left(1-2 u_{3}\right) P_{j-j_{1}-j_{2}}^{\left(2 j_{1}-1,2 j_{2}-1\right)}\left(\frac{u_{1}-u_{2}}{1-u_{3}}\right) .
$$

Note that this basis of $Y_{J j}^{(12) 3}\left(u_{i}\right)$ can be related to the basis of $Y_{J j}^{1(23)}\left(u_{i}\right)$ (or $Y_{J j}^{2(31)}\left(u_{i}\right)$ ) through the Racah $6 j$-symbols of $S L(2, \mathbb{R})$ group [22, 2].

For axial-vector mesons, the three-parton LCDAs correspond to the higher Fock states consist of the quark, anti-quark, and gluon. We refer to $j_{1}=1, j_{2}=1$ and $j_{3}=3 / 2$ as the conformal spins of the quark, anti-quark and gluon, respectively, in an axial-vector meson. Using Eq. (16), we redefine the orthonormal basis functions,

$$
J_{N n}^{(12) 3}\left(u_{i}\right)=\frac{(-1)^{N}}{2} Y_{J j}^{(12) 3}\left(u_{i}\right)=\frac{(-1)^{N}}{2}\left(1-u_{3}\right)^{n} P_{N-n}^{(2,3+2 n)}\left(1-2 u_{3}\right) P_{n}^{(1,1)}\left(\frac{u_{1}-u_{2}}{1-u_{3}}\right),
$$

where $J$ is the total three-parton conformal spin, $j$ is the total conformal spin of the quark and anti-quark, $N=J-7 / 2$ and $n=j-2$ with $N, n=0,1, \cdots$, and $N \geq n$. Thus, the meson's three-parton distribution amplitudes of twist-three can be represented as

$$
\phi^{\bar{q} q g}\left(u_{i}\right)=360 u_{1} u_{2} u_{3}^{2} \sum_{N=0}^{\infty} \sum_{n=0}^{N} \omega_{N-n, n} J_{N n}^{(12) 3}\left(u_{i}\right) \text {. }
$$


If one takes into account the conformal spins of the distribution amplitudes up to order of $J=9 / 2$, the twist-3 LCDA reads 2

$$
\begin{aligned}
\phi^{\bar{q} q g}\left(u_{i}\right) & \cong 360 u_{1} u_{2} u_{3}^{2}\left[\omega_{0,0} J_{00}^{(12) 3}\left(u_{i}\right)+\omega_{1,0} J_{10}^{(12) 3}\left(u_{i}\right)+\omega_{0,1} J_{11}^{(12) 3}\left(u_{i}\right)\right] \\
& =360 u_{1} u_{2} u_{3}^{2}\left[\frac{1}{2} \omega_{0,0}+\frac{1}{2}\left(7 u_{3}-3\right) \omega_{1,0}-\left(u_{1}-u_{2}\right) \omega_{0,1}\right]
\end{aligned}
$$

\section{Definitions of light-cone distribution amplitudes}

In what follows, we define $z=y-x$ with $z^{2}=0$, and introduce the light-like vector $p_{\mu}=P_{\mu}-m_{A}^{2} z_{\mu} /(2 P \cdot z)$ with the axial-vector meson's momentum $P^{2}=m_{A}^{2}$. The polarization vector $\epsilon_{\mu}^{(\lambda)}$ of the axial-vector meson can be decomposed into longitudinal and transverse projections as [9, 10]

$$
\epsilon_{\| \mu}^{(\lambda)} \equiv \frac{\epsilon^{(\lambda)} \cdot z}{p \cdot z}\left(p_{\mu}-\frac{m_{A}^{2}}{2 p \cdot z} z_{\mu}\right), \quad \epsilon_{\perp \mu}^{(\lambda)}=\epsilon_{\mu}^{(\lambda)}-\epsilon_{\| \mu}^{(\lambda)},
$$

respectively. In QCD description of hard processes involving axial vector mesons, one encounter bilocal operators sandwiched between the vacuum and the meson,

$$
\left\langle A(P, \lambda)\left|\bar{q}_{1}(y) \Gamma[y, x] q_{2}(x)\right| 0\right\rangle
$$

where $\Gamma$ is a generic notation for the Dirac matrix structure and the path-ordered gauge factor is

$$
[y, x]=\mathrm{P} \exp \left[i g_{s} \int_{0}^{1} d t(x-y)_{\mu} A^{\mu}(t x+(1-t) y)\right] .
$$

This factor is equal to unity in the light-cone gauge which is equivalent to the fixedpoint gauge $(x-y)^{\mu} A_{\mu}(x-y)=0$, which is also called the Fock-Schwinger gauge, as the quark-antiquark pair is at the light-like separation. For simplicity, here and below we do not show the gauge factor. For $f_{1}$ and $h_{1}$, the operators in Eq. (24) correspond to

\footnotetext{
${ }^{2}$ The contribution of conformal spin $11 / 2$ to the meson's three-parton distribution amplitudes of twistthree is

$$
\begin{aligned}
& 360 u_{1} u_{2} u_{3}^{2}\left[\omega_{2,0} J_{20}^{(12) 3}\left(u_{i}\right)+\omega_{1,1} J_{21}^{(12) 3}\left(u_{i}\right)+\omega_{0,2} J_{22}^{(12) 3}\left(u_{i}\right)\right] \\
= & 360 u_{1} u_{2} u_{3}^{2}\left[\left(18 u_{3}^{2}-16 u_{3}+3\right) \omega_{2,0}+3\left(1-3 u_{3}\right)\left(u_{1}-u_{2}\right) \omega_{1,1}+\frac{3}{2}\left[\left(1-u_{3}\right)^{2}-5 u_{1} u_{2}\right] \omega_{0,2}\right] .
\end{aligned}
$$
}

Comparing the above result with that given by Braun and Filyanov [1], we thus find

$$
\begin{aligned}
& \omega_{2,0}=\frac{2}{5} \omega_{2,0}^{\mathrm{BF}}+\frac{1}{5} \omega_{1,1}^{\mathrm{BF}}, \\
& \omega_{0,2}=\frac{8}{15} \omega_{2,0}^{\mathrm{BF}}-\frac{2}{5} \omega_{1,1}^{\mathrm{BF}} .
\end{aligned}
$$

Note that for $\omega_{1,1}$ there is no corresponding term given in Ref. [1]. 
$(\bar{u}(y) \Gamma u(x)+\bar{d}(y) \Gamma d(x)+\bar{s}(y) \Gamma s(x)) / \sqrt{3}$, while for $f_{8}$ and $h_{8}$, the relevant forms of operators are $(\bar{u}(y) \Gamma u(x)+\bar{d}(y) \Gamma d(x)-2 \bar{s}(y) \Gamma s(x)) / \sqrt{6}$. In the present study, we adopt the conventions $D_{\alpha}=\partial_{\alpha}+i g_{s} A_{\alpha}^{a} \lambda^{a} / 2, \widetilde{G}_{\alpha \beta}=(1 / 2) \epsilon_{\alpha \beta \mu \nu} G^{\mu \nu}, \epsilon^{0123}=-1$.

In general, the LCDAs are scheme- and scale-dependent. One can catalog the distribution amplitudes into two classes: (i) chiral-even LCDAs for which the relevant current operators involve chirality-conserving structures $\Gamma=\left\{\gamma_{\mu}, \gamma_{\mu} \gamma_{5}\right\}$, and (ii) chiral-odd LCDAs for which the operators contain chirality-violating structures $\Gamma=\left\{\sigma_{\mu \nu}\left(\gamma_{5}\right), 1\right\}$.

\subsection{Two-parton distribution amplitudes}

The chiral-even LCDAs are given by 3

$$
\begin{aligned}
\left\langle A(P, \lambda)\left|\bar{q}_{1}(y) \gamma_{\mu} \gamma_{5} q_{2}(x)\right| 0\right\rangle= & i f_{A} m_{A} \int_{0}^{1} d u e^{i(u p y+\bar{u} p x)}\left\{p_{\mu} \frac{\epsilon^{*(\lambda)} z}{p z} \Phi_{\|}(u)+\epsilon_{\perp \mu}^{*(\lambda)} g_{\perp}^{(a)}(u)\right. \\
& \left.-\frac{1}{2} z_{\mu} \frac{\epsilon^{*(\lambda)} z}{(p z)^{2}} m_{A}^{2} g_{3}(u)\right\} \\
\left\langle A(P, \lambda)\left|\bar{q}_{1}(y) \gamma_{\mu} q_{2}(x)\right| 0\right\rangle= & -i f_{A} m_{A} \epsilon_{\mu \nu \rho \sigma} \epsilon_{(\lambda)}^{* \nu} p^{\rho} z^{\sigma} \int_{0}^{1} d u e^{i(u p y+\bar{u} p x)} \frac{g_{\perp}^{(v)}(u)}{4}
\end{aligned}
$$

where $u$ and $\bar{u} \equiv 1-u$ are the momentum fractions of $q_{1}$ and $\bar{q}_{2}$ in the axial-vector meson, respectively. The chiral-odd LCDAs are defined by 4

$$
\begin{aligned}
\left\langle A(P, \lambda)\left|\bar{q}_{1}(y) \sigma_{\mu \nu} \gamma_{5} q_{2}(x)\right| 0\right\rangle & =f_{A}^{\perp} \int_{0}^{1} d u e^{i(u p y+\bar{u} p x)}\left\{\left(\epsilon_{\perp \mu}^{*(\lambda)} p_{\nu}-\epsilon_{\perp \nu}^{*(\lambda)} p_{\mu}\right) \Phi_{\perp}(u)\right. \\
& +\frac{m_{A}^{2} \epsilon^{*(\lambda)} z}{(p z)^{2}}\left(p_{\mu} z_{\nu}-p_{\nu} z_{\mu}\right) h_{\|}^{(t)}(u) \\
& \left.+\frac{1}{2}\left(\epsilon_{\perp \mu}^{*(\lambda)} z_{\nu}-\epsilon_{\perp \nu}^{*(\lambda)} z_{\mu}\right) \frac{m_{A}^{2}}{p \cdot z} h_{3}(u)\right\} \\
\left\langle A(P, \lambda)\left|\bar{q}_{1}(y) \gamma_{5} q_{2}(x)\right| 0\right\rangle= & f_{A}^{\perp} m_{A}^{2}\left(\epsilon^{*(\lambda)} z\right) \int_{0}^{1} d u e^{i(u p y+\bar{u} p x)} \frac{h_{\|}^{(p)}(u)}{2} .
\end{aligned}
$$

Here $\Phi_{\|}, \Phi_{\perp}$ are of twist-2, $g_{\perp}^{(a)}, g_{\perp}^{(v)}, h_{\|}^{(t)}, h_{\|}^{(p)}$ of twist-3, and $g_{3}, h_{3}$ of twist-4. In SU(3) limit, due to G-parity, $\Phi_{\|}, g_{\perp}^{(a)}, g_{\perp}^{(v)}$, and $g_{3}$ are symmetric [antisymmetric] under the replacement $u \rightarrow 1-u$ for the $1^{3} P_{1}\left[1^{1} P_{1}\right]$ states, whereas $\Phi_{\perp}, h_{\|}^{(t)}, h_{\|}^{(p)}$, and $h_{3}$ are antisymmetric [symmetric]. In other words, in the $\mathrm{SU}(3)$ limit it follows that

$$
\int_{0}^{1} d u \Phi_{\perp}(u)=\int_{0}^{1} d u h_{\|}^{(t)}(u)=\int_{0}^{1} d u h_{\|}^{(p)}(u)=\int_{0}^{1} d u h_{3}(u)=0
$$

${ }^{3} g_{\perp}^{(v)}$ and $g_{\perp}^{(a)}$ given in Ref. [18] is respectively redefined to be $g_{\perp}^{(a)}$ and $g_{\perp}^{(v)}$ in this paper.

${ }^{4} h_{\|}^{(s)}$ given in Ref. [18] is redefined to be $h_{\|}^{(p)}$ in this paper. 
for $1^{3} P_{1}$ states, but becomes

$$
\int_{0}^{1} d u \Phi_{\|}(u)=\int_{0}^{1} d u g_{\perp}^{(a)}(u)=\int_{0}^{1} d u g_{\perp}^{(v)}(u)=\int_{0}^{1} d u g_{3}(u)=0
$$

for $1^{1} P_{1}$ states. The above integrals are not zero if $m_{q_{1}} \neq m_{q_{2}}$, and the detailed results are summarized in Secs. 6.1 and 6.2. We will not further discuss the twist-4 LCDAs, $g_{3}$ and $h_{3}$, below. For convenience, we therefore normalize the distribution amplitudes of the $1^{3} P_{1}$ $\left[1^{1} P_{1}\right]$ states to be subject to

$$
\int_{0}^{1} d u \Phi_{\|}(u)=1 \quad\left[\int_{0}^{1} d u \Phi_{\perp}(u)=1\right]
$$

and take $f_{{ }^{3} P_{1}}^{\perp}=f_{{ }^{3} P_{1}} \quad\left[f_{{ }^{1} P_{1}}=f_{{ }_{1} P_{1}}^{\perp}(\mu=1 \mathrm{GeV})\right]$ in the study, such that we have

$$
\begin{aligned}
& \left\langle 1^{3} P_{1}(P, \lambda)\left|\bar{q}_{1}(0) \sigma_{\mu \nu} \gamma_{5} q_{2}(0)\right| 0\right\rangle=f_{3 P_{1}}^{\perp} a_{0}^{\perp,}{ }^{3} P_{1}\left(\epsilon_{\mu}^{*(\lambda)} P_{\nu}-\epsilon_{\nu}^{*(\lambda)} P_{\mu}\right), \\
& \left\langle 1^{1} P_{1}(P, \lambda)\left|\bar{q}_{1}(0) \gamma_{\mu} \gamma_{5} q_{2}(0)\right| 0\right\rangle=i f_{1} P_{1} a_{0}^{\|,{ }^{1} P_{1}} m_{1} P_{1} \epsilon_{\mu}^{*(\lambda)}
\end{aligned}
$$

where $a_{0}^{\perp,}{ }^{3} P_{1}$ and $a_{0}^{\|,}{ }^{1} P_{1}$ are the Gegenbauer zeroth moments, defined in Eqs. (60) and (48), and vanish in the SU(3) limit. Using the results given in Eqs. (11), (12) and (14), the twoparton distribution amplitudes can be expanded in a series of partial waves with different conformal spins. However, in analogy to that discussed in Refs. [1, 9], by means of QCD EOMs, $g_{\perp}^{(a)}, g_{\perp}^{(v)}, h_{\|}^{(t)}$, and $h_{\|}^{(p)}$ can be expressed in terms of twist-two two-parton and twistthree three-parton distribution amplitudes. The detailed results are given in Secs. 3.3 and 3.4. It should be stressed that EOMs only relate terms with the same conformal spin so that the relations between LCDAs can be satisfied order by order in the conformal expansion [2].

\subsection{Three-parton distribution amplitudes of twist-three}

The three-parton chiral-even $(\mathcal{A}, \mathcal{V})$ and chiral-odd $(\mathcal{T})$ distribution amplitudes of twist-3 are defined by

$$
\begin{aligned}
& \left\langle A(P, \lambda)\left|\bar{q}_{1}(-x) \gamma_{\alpha} \gamma_{5} g_{s} G_{\mu \nu}(v x) q_{2}(x)\right| 0\right\rangle=-p_{\alpha}\left[p_{\mu} \epsilon_{\perp \nu}^{*(\lambda)}-p_{\nu} \epsilon_{\perp \mu}^{*(\lambda)}\right] f_{3 A}^{A} \mathcal{A}(v,-p x)+\ldots \\
& \left\langle A(P, \lambda)\left|\bar{q}_{1}(-x) \gamma_{\alpha} g_{s} \widetilde{G}_{\mu \nu}(v x) q_{2}(x)\right| 0\right\rangle=i p_{\alpha}\left[p_{\mu} \epsilon_{\perp \nu}^{*(\lambda)}-p_{\nu} \epsilon_{\perp \mu}^{*(\lambda)}\right] f_{3 A}^{V} \mathcal{V}(v,-p x)+\ldots \\
& \left\langle A(P, \lambda)\left|\bar{q}_{1}(-x) \sigma_{\alpha \beta} \gamma_{5} g_{s} G_{\mu \nu}(v x) q_{2}(x)\right| 0\right\rangle \\
& \quad=-i \frac{\epsilon^{*(\lambda)} x}{2(p x)}\left[p_{\alpha} p_{\mu} g_{\beta \nu}^{\perp}-p_{\beta} p_{\mu} g_{\alpha \nu}^{\perp}-p_{\alpha} p_{\nu} g_{\beta \mu}^{\perp}+p_{\beta} p_{\nu} g_{\alpha \mu}^{\perp}\right] f_{3 A}^{\perp} m_{A} \mathcal{T}(v,-p x)+\ldots
\end{aligned}
$$

where the ellipses stand for terms of twist higher than three, the following shorthand notations are used:

$$
\mathcal{A}(v,-p x) \equiv \int \mathcal{D} \underline{\alpha} e^{i p x\left(\alpha_{q_{2}}-\alpha_{q_{1}}+v \alpha_{g}\right)} \mathcal{A}\left(\alpha_{q_{1}} \alpha_{q_{2}}, \alpha_{g}\right)
$$


etc., and the integration measure is defined as

$$
\int \mathcal{D} \underline{\alpha} \equiv \int_{0}^{1} d \alpha_{q_{1}} \int_{0}^{1} d \alpha_{q_{2}} \int_{0}^{1} d \alpha_{g} \delta\left(1-\sum \alpha_{i}\right) .
$$

Here $\alpha_{q_{1}}, \alpha_{q_{2}}$, and $\alpha_{g}$ are the respective momentum fractions carried by $q_{1}, \bar{q}_{2}$ quarks and gluon in the axial-vector meson. Due to G-parity, for a $1^{3} P_{1}\left[1^{1} P_{1}\right]$ state $\mathcal{A}$ is antisymmetric [symmetric] under the interchange $\alpha_{q_{2}} \leftrightarrow \alpha_{q_{1}}$ in the $\mathrm{SU}(3)$ limit, while $\mathcal{V}$ and $\mathcal{T}$ are symmetric [antisymmetric] (cf. the case of the $\rho$ meson in Ref. [9]). Taking into account the contributions up to terms of conformal spin $9 / 2$ and considering the corrections which arise from the quark masses, the distribution amplitudes can be approximately written as

$$
\begin{aligned}
& \mathcal{A}=5040\left(\alpha_{q_{1}}-\alpha_{q_{2}}\right) \alpha_{q_{1}} \alpha_{q_{2}} \alpha_{g}^{2}+360 \alpha_{q_{1}} \alpha_{q_{2}} \alpha_{g}^{2}\left[\lambda_{3 P_{1}}^{A}+\sigma_{3 P_{1}}^{A} \frac{1}{2}\left(7 \alpha_{g}-3\right)\right] \\
& \mathcal{V}=360 \alpha_{q_{1}} \alpha_{q_{2}} \alpha_{g}^{2}\left[1+\omega_{3 P_{1}}^{V} \frac{1}{2}\left(7 \alpha_{g}-3\right)\right]+5040\left(\alpha_{q_{1}}-\alpha_{q_{2}}\right) \alpha_{q_{1}} \alpha_{q_{2}} \alpha_{g}^{2} \sigma_{3 P_{1}}^{V}, \\
& \mathcal{T}=360 \alpha_{q_{1}} \alpha_{q_{2}} \alpha_{g}^{2}\left[1+\omega_{3 P_{1}}^{\perp} \frac{1}{2}\left(7 \alpha_{g}-3\right)\right]+5040\left(\alpha_{q_{1}}-\alpha_{q_{2}}\right) \alpha_{q_{1}} \alpha_{q_{2}} \alpha_{g}^{2} \sigma_{3}^{\perp} P_{1},
\end{aligned}
$$

for the $1^{3} P_{1}$ states, and

$$
\begin{aligned}
& \mathcal{A}=360 \alpha_{q_{1}} \alpha_{q_{2}} \alpha_{g}^{2}\left[1+\omega_{1 P_{1}}^{A} \frac{1}{2}\left(7 \alpha_{g}-3\right)\right]+5040\left(\alpha_{q_{1}}-\alpha_{q_{2}}\right) \alpha_{q_{1}} \alpha_{q_{2}} \alpha_{g}^{2} \sigma_{P_{1}}^{A}, \\
& \mathcal{V}=5040\left(\alpha_{q_{1}}-\alpha_{q_{2}}\right) \alpha_{q_{1}} \alpha_{q_{2}} \alpha_{g}^{2}+360 \alpha_{q_{1}} \alpha_{q_{2}} \alpha_{g}^{2}\left[\lambda_{1_{P_{1}}}^{V}+\sigma_{P_{1}}^{V} \frac{1}{2}\left(7 \alpha_{g}-3\right)\right], \\
& \mathcal{T}=5040\left(\alpha_{q_{1}}-\alpha_{q_{2}}\right) \alpha_{q_{1}} \alpha_{q_{2}} \alpha_{g}^{2}+360 \alpha_{q_{1}} \alpha_{q_{2}} \alpha_{g}^{2}\left[\lambda^{\perp} P_{1}+\sigma_{{ }_{1}}^{\perp} \frac{1}{2}\left(7 \alpha_{g}-3\right)\right],
\end{aligned}
$$

for the $1^{1} P_{1}$ states, where $\lambda$ 's correspond to conformal spin $7 / 2$, while $\omega$ 's and $\sigma$ 's are parameters with conformal spin 9/2. As the $\mathrm{SU}(3)$-symmetry (and G-parity) is restored, we have $\lambda^{\prime} \mathrm{s}=\sigma^{\prime} \mathrm{s}=0$. The normalization constants $f_{3 A}^{A}, f_{3 A}^{V}$ and $f_{3 A}^{\perp}$ are thus defined in such a way that

$$
\begin{aligned}
\int \mathcal{D} \underline{\alpha}\left(\alpha_{q_{1}}-\alpha_{q_{2}}\right) \mathcal{A}\left(\alpha_{q_{1}}, \alpha_{q_{2}}, \alpha_{g}\right) & =1, \quad \int \mathcal{D} \underline{\alpha} \mathcal{A}\left(\alpha_{q_{1}}, \alpha_{q_{2}}, \alpha_{g}\right)=\lambda_{3 P_{1}}^{A}, \\
\int \mathcal{D} \underline{\alpha} \mathcal{V}\left(\alpha_{q_{1}}, \alpha_{q_{2}}, \alpha_{g}\right) & =1, \quad \int \mathcal{D} \underline{\alpha}\left(\alpha_{q_{1}}-\alpha_{q_{2}}\right) \mathcal{V}\left(\alpha_{q_{1}}, \alpha_{q_{2}}, \alpha_{g}\right)=\sigma_{3 P_{1}}^{V} \\
\int \mathcal{D} \underline{\alpha} \mathcal{T}\left(\alpha_{q_{1}}, \alpha_{q_{2}}, \alpha_{g}\right) & =1, \quad \int \mathcal{D} \underline{\alpha}\left(\alpha_{q_{1}}-\alpha_{q_{2}}\right) \mathcal{T}\left(\alpha_{q_{1}}, \alpha_{q_{2}}, \alpha_{g}\right)=\sigma_{3}^{\perp},
\end{aligned}
$$

for the $1^{3} P_{1}$ states, and

$$
\begin{aligned}
\int \mathcal{D} \underline{\alpha} \mathcal{A}\left(\alpha_{q_{1}}, \alpha_{q_{2}}, \alpha_{g}\right) & =1, \quad \int \mathcal{D} \underline{\alpha}\left(\alpha_{q_{1}}-\alpha_{q_{2}}\right) \mathcal{A}\left(\alpha_{q_{1}}, \alpha_{q_{2}}, \alpha_{g}\right)=\sigma_{P_{1}}^{A}, \\
\int \mathcal{D} \underline{\alpha}\left(\alpha_{q_{1}}-\alpha_{q_{2}}\right) \mathcal{V}\left(\alpha_{q_{1}}, \alpha_{q_{2}}, \alpha_{g}\right) & =1, \quad \int \mathcal{D} \underline{\alpha} \mathcal{V}\left(\alpha_{q_{1}}, \alpha_{q_{2}}, \alpha_{g}\right)=\lambda_{{ }_{P}}^{V}, \\
\int \mathcal{D} \underline{\alpha}\left(\alpha_{q_{1}}-\alpha_{q_{2}}\right) \mathcal{T}\left(\alpha_{q_{1}}, \alpha_{q_{2}}, \alpha_{g}\right) & =1, \quad \int \mathcal{D} \underline{\alpha} \mathcal{T}\left(\alpha_{q_{1}}, \alpha_{q_{2}}, \alpha_{g}\right)=\lambda_{{ }^{1} P_{1}}^{\perp},
\end{aligned}
$$


for the $1^{1} P_{1}$ states.

\subsection{Relations among chiral-even LCDAs}

$\Phi_{\|}(u, \mu)$ can be expanded in a series of Gegenbauer polynomials [1, 2]:

$$
\Phi_{\|}^{A}(u, \mu)=6 u(1-u)\left[a_{0}^{\|, A}+\sum_{l=1}^{\infty} a_{l}^{\|, A}(\mu) C_{l}^{3 / 2}(2 u-1)\right],
$$

where $\mu$ is the normalization scale and the multiplicatively renormalizable coefficients (or called Gegenbauer moments) are:

$$
a_{l}^{\|, A}(\mu)=\frac{2(2 l+3)}{3(l+1)(l+2)} \int_{0}^{1} d x C_{l}^{3 / 2}(2 x-1) \Phi_{\|}^{A}(x, \mu) .
$$

In the limit of $m_{q_{1}}=m_{q_{2}}$, only terms with even (odd) $l$ survive due to G-parity invariance for the $1^{3} P_{1}\left(1^{1} P_{1}\right)$ mesons. In the expansion of $\Phi_{\|}^{A}(u, \mu)$ in Eq. (48), the conformal invariance of the light-cone QCD exhibits that partial waves with different conformal spin cannot mix under renormalization to leading-order accuracy. As a consequence, the Gegenbauer moments $a_{l}^{\|}$renormalize multiplicatively:

$$
a_{l}^{\|, A}(\mu)=a_{l}^{\|, A}\left(\mu_{0}\right)\left(\frac{\alpha_{s}\left(\mu_{0}\right)}{\alpha_{s}(\mu)}\right)^{-\gamma_{(l)}^{\|} / b},
$$

where $b=\left(11 N_{c}-2 n_{f}\right) / 3$ and the one-loop anomalous dimensions are [27]

$$
\gamma_{(l)}^{\|}=C_{F}\left(1-\frac{2}{(l+1)(l+2)}+4 \sum_{j=2}^{l+1} \frac{1}{j}\right),
$$

with $C_{F}=\left(N_{c}^{2}-1\right) /\left(2 N_{c}\right)$.

Applying the QCD equations of motion, as the case for the vector mesons in Refs. [9, 10], one can obtain some useful nonlocal operator identities (see Appendix B) such that the two-parton distribution amplitudes $g_{\perp}^{(a)}$ and $g_{\perp}^{(v)}$ can be represented in terms of $\Phi_{\|, \perp}$ and three-parton distribution amplitudes. Setting $y=-x$ and adapting the formulas derived in Ref. [9] for vector mesons to the present case, we find 5

$$
\begin{aligned}
\int_{0}^{1} d u e^{-i \xi p x} g_{\perp}^{(a)}(u)=\int_{0}^{1} d t \int_{0}^{1} d u e^{-i t \xi p x} \Phi_{\|}(u) \\
-\zeta_{3, A}^{V}(p x)^{2} \int_{0}^{1} t^{2} d t \int_{-1}^{1} d v \mathcal{V}(v,-t p x)-\zeta_{3, A}^{A}(p x)^{2} \int_{0}^{1} d t t^{2} \int_{-1}^{1} d v v \mathcal{A}(v,-t p x) \\
\quad-\frac{1}{2}(p x)^{2} \int_{0}^{1} d t t^{2} \int_{0}^{1} d u e^{-i t \xi p x} g_{\perp}^{(v)}(u)-i \widetilde{\delta}_{+}(p x) \int_{0}^{1} d t t \int_{0}^{1} d u e^{-i t \xi p x} \Phi_{\perp}(u), \quad(52)
\end{aligned}
$$

\footnotetext{
${ }^{5}$ Here and below we use the notations close to that given in Ref. [9].
} 
and

$$
\begin{gathered}
\frac{1}{2} \int_{0}^{1} d u e^{-i \xi p x} g_{\perp}^{(v)}(u)=\int_{0}^{1} d t t \int_{0}^{1} d u e^{-i t \xi p x} g_{\perp}^{(a)}(u) \\
-i \zeta_{3, A}^{A}(p x) \int_{0}^{1} d t t^{2} \int_{-1}^{1} d v \mathcal{A}(v,-t p x)-i \zeta_{3, A}^{V}(p x) \int_{0}^{1} d t t^{2} \int_{-1}^{1} d v v \mathcal{V}(v,-t p x) \\
+\widetilde{\delta}_{-} \int_{0}^{1} d t t \int_{0}^{1} d u e^{-i t \xi p x} \Phi_{\perp}(u)
\end{gathered}
$$

where $\xi \equiv 2 u-1$, and we introduced the abbreviation

$$
\widetilde{\delta}_{ \pm}=\frac{f_{A}^{\perp}}{f_{A}} \frac{m_{q_{2}} \pm m_{q_{1}}}{m_{A}}, \quad \zeta_{3, A}^{V, A}=\frac{f_{3 A}^{V, A}}{f_{A} m_{A}}
$$

Solving Eqs. (52) and (53), we obtain the solutions [9] for $g_{\perp}^{(v)}(u)$ :

$$
g_{\perp}^{(v)}(u)=\bar{u} \int_{0}^{u} d v \frac{1}{\bar{v}} \Psi(v)+u \int_{u}^{1} d v \frac{1}{v} \Psi(v) .
$$

and for $g_{\perp}^{(a)}(u)$ :

$$
\begin{aligned}
g_{\perp}^{(a)}(u)= & \frac{1}{4}\left[\int_{0}^{u} d v \frac{1}{\bar{v}} \Psi(v)+\int_{u}^{1} d v \frac{1}{v} \Psi(v)\right]-\widetilde{\delta}_{-} \Phi_{\perp}(u) \\
& +\zeta_{3, A}^{V} \int_{0}^{u} d \alpha_{q_{1}} \int_{0}^{u} d \alpha_{q_{2}} \frac{1}{1-\alpha_{q_{1}}-\alpha_{q_{2}}}\left(\frac{d}{d \alpha_{q_{1}}}+\frac{d}{d \alpha_{q_{2}}}\right) \mathcal{V}(\underline{\alpha}) \\
& +\zeta_{3, A}^{A} \frac{d}{d u} \int_{0}^{u} d \alpha_{q_{1}} \int_{0}^{\bar{u}} d \alpha_{q_{2}} \frac{\mathcal{A}(\underline{\alpha})}{1-\alpha_{q_{1}}-\alpha_{q_{2}}},
\end{aligned}
$$

where

$$
\begin{aligned}
\Psi(u)= & 2 \Phi_{\|}(u)-\widetilde{\delta}_{-} \xi \Phi_{\perp}^{\prime}(u)-\widetilde{\delta}_{+} \Phi_{\perp}^{\prime}(u) \\
& +2 \zeta_{3, A}^{A} \frac{d}{d u} \int_{0}^{u} d \alpha_{q_{1}} \int_{0}^{\bar{u}} d \alpha_{q_{2}} \frac{1}{1-\alpha_{q_{1}}-\alpha_{q_{2}}}\left(\alpha_{q_{1}} \frac{d}{d \alpha_{q_{1}}}+\alpha_{q_{2}} \frac{d}{d \alpha_{q_{2}}}\right) \mathcal{A}(\underline{\alpha}) \\
& +2 \zeta_{3, A}^{V} \frac{d}{d u} \int_{0}^{u} d \alpha_{q_{1}} \int_{0}^{\bar{u}} d \alpha_{q_{2}} \frac{1}{1-\alpha_{q_{1}}-\alpha_{q_{2}}}\left(\alpha_{q_{1}} \frac{d}{d \alpha_{q_{1}}}-\alpha_{q_{2}} \frac{d}{d \alpha_{q_{2}}}\right) \mathcal{V}(\underline{\alpha}) .
\end{aligned}
$$


Neglecting the three-parton distribution amplitudes containing gluons and terms proportional to light quark masses, $g_{\perp}^{(a)}$ and $g_{\perp}^{(v)}$ are thus related to the twist-2 ones by WandzuraWilczek-type relations:

$$
\begin{aligned}
& g_{\perp}^{(a) W W}(u) \simeq \frac{1}{2}\left[\int_{0}^{u} d v \frac{1}{\bar{v}} \Phi_{\|}(v)+\int_{u}^{1} d v \frac{1}{v} \Phi_{\|}(v)\right] \\
& g_{\perp}^{(v) W W}(u) \simeq 2 \bar{u} \int_{0}^{u} d v \frac{1}{\bar{v}} \Phi_{\|}(v)+2 u \int_{u}^{1} d v \frac{1}{v} \Phi_{\|}(v) .
\end{aligned}
$$

\subsection{Relations among chiral-odd LCDAs}

The leading-twist LCDAs $\Phi_{\perp}^{A}(u, \mu)$ can be expanded as [1, 2]

$$
\Phi_{\perp}^{A}(u, \mu)=6 u(1-u)\left[a_{0}^{\perp}+\sum_{l=1}^{\infty} a_{l}^{\perp, A}(\mu) C_{l}^{3 / 2}(2 u-1)\right],
$$

where the multiplicatively renormalizable Gegenbauer moments, in analogy to Eq. (49), read

$$
a_{l}^{\perp, A}(\mu)=\frac{2(2 l+3)}{3(l+1)(l+2)} \int_{0}^{1} d x C_{l}^{3 / 2}(2 x-1) \Phi_{\perp}^{A}(x, \mu),
$$

which satisfy

$$
\left(f_{A}^{\perp} a_{l}^{\perp, A}\right)(\mu)=\left(f_{A}^{\perp} a_{l}^{\perp, A}\right)\left(\mu_{0}\right)\left(\frac{\alpha_{s}\left(\mu_{0}\right)}{\alpha_{s}(\mu)}\right)^{-\gamma\left(\frac{1}{(l)} / b\right.}
$$

with the one-loop anomalous dimensions being [27]

$$
\gamma_{(l)}^{\perp}=C_{F}\left(1+4 \sum_{j=2}^{l+1} \frac{1}{j}\right)
$$

In the limit of $m_{q_{1}}=m_{q_{2}}, a_{l}^{\perp, A}$ with even (odd) $l$ vanish due to G-parity invariance for the $1^{3} P_{1}\left(1^{1} P_{1}\right)$ mesons.

In analogy to Eqs. (52) and (52), using the formulas developed in Ref. [9] for vector mesons to the present case, we obtain the integral equations:

$$
\begin{gathered}
i p x \int_{0}^{1} d u e^{-i \xi p x} \xi h_{\|}^{(t)}(u)-2 \int_{0}^{1} d u e^{-i \xi p x}\left(h_{\|}^{(t)}(u)-\Phi_{\perp}(u)\right) \\
=\zeta_{3, A}^{\perp}(p x)^{2} \int_{-1}^{1} d v v \mathcal{T}(v,-p x)+(p x)^{2} \int_{0}^{1} e^{-i \xi p x} h_{\|}^{(p)}(u)+i \delta_{+} p x \int_{0}^{1} d u e^{-i \xi p x} \Phi_{\|}(u), \\
\int_{0}^{1} d u e^{-i \xi p x} h_{\|}^{(p)}(u)=-i \zeta_{3, A}^{\perp} p x \int_{0}^{1} t d t \int_{-1}^{1} d v \mathcal{T}(v,-t p x)+\int_{0}^{1} d t \int_{0}^{1} d u e^{-i \xi t p x} h_{\|}^{(t)}(u) \\
+\delta_{-} \int_{0}^{1} d t \int_{0}^{1} d u e^{-i \xi t p x} \Phi_{\|}(u),
\end{gathered}
$$


where we have introduced the notations

$$
\delta_{ \pm}=\frac{f_{A}}{f_{A}^{\perp}} \frac{m_{q_{2}} \pm m_{q_{1}}}{m_{A}}, \quad \zeta_{3, A}^{\perp}=\frac{f_{3, A}^{\perp}}{f_{A}^{\perp} m_{A}} .
$$

The solutions of the integral equations [9] given in Eqs. (64) and (65) can be obtained to be

$$
h_{\|}^{(p)}(u)=\bar{u} \int_{0}^{u} d v \frac{1}{\bar{v}} \Theta(v)+u \int_{u}^{1} d v \frac{1}{v} \Theta(v),
$$

and

$$
\begin{aligned}
h_{\|}^{(t)}(u)= & \frac{1}{2} \xi\left(\int_{0}^{u} d v \frac{1}{\bar{v}} \Theta(v)-\int_{u}^{1} d v \frac{1}{v} \Theta(v)\right)-\delta_{-} \Phi_{\|}(u) \\
& +\zeta_{3, A}^{\perp} \frac{d}{d u} \int_{0}^{u} d \alpha_{q_{1}} \int_{0}^{\bar{u}} d \alpha_{q_{2}} \frac{1}{1-\alpha_{q_{2}}-\alpha_{q_{1}}} \mathcal{T}(\underline{\alpha}),
\end{aligned}
$$

where

$$
\begin{aligned}
\Theta(u)= & 2 \Phi_{\perp}(u)+\delta_{-}\left(\Phi_{\|}(u)-\frac{1}{2} \xi \Phi_{\|}^{\prime}(u)\right)-\frac{1}{2} \delta_{+} \Phi_{\|}^{\prime}(u) \\
& +\zeta_{3, A}^{\perp} \frac{d}{d u} \int_{0}^{u} d \alpha_{q_{1}} \int_{0}^{\bar{u}} d \alpha_{q_{2}} \frac{1}{1-\alpha_{q_{2}}-\alpha_{q_{1}}}\left(\alpha_{q_{1}} \frac{d}{d \alpha_{q_{1}}}+\alpha_{q_{2}} \frac{d}{d \alpha_{q_{2}}}-1\right) \mathcal{T}(\underline{\alpha}) .
\end{aligned}
$$

The two-parton twist-3 distribution amplitudes are thus related to the twist-2 ones approximately by Wandzura-Wilczek-type relations

$$
\begin{aligned}
& h_{\|}^{(t) W W}(u)=\xi\left(\int_{0}^{u} d v \frac{\Phi_{\perp}(v)}{\bar{v}}-\int_{u}^{1} d v \frac{\Phi_{\perp}(v)}{v}\right) \\
& h_{\|}^{(p) W W}(u)=2\left(\bar{u} \int_{0}^{u} d v \frac{\Phi_{\perp}(v)}{\bar{v}}+u \int_{u}^{1} d v \frac{\Phi_{\perp}(v)}{v}\right) .
\end{aligned}
$$

\subsection{Comparison of the vector mesons and axial-vector mesons}

In comparison with the vector mesons, we summarize in Table 1 the (anti-)symmetric properties of LCDAs of axial-vector mesons in the SU(3) limit under the interchange of the momentum fractions of the quark and antiquark. On the other hand, comparing results for axial-vector mesons given in Eqs. (26) $-(29)$, (5) vector mesons given in Ref. [9], we can see an analogy between the two cases. One can relate the mathematical forms of two-parton LCDAs of twist-3 for vector mesons and axial-vector mesons in the following way: 
with the replacement

$$
\begin{array}{ccc}
\text { vector mesons : } & & \text { axial-vector mesons : } \\
g_{\perp}^{(v)} & \longleftrightarrow & g_{\perp}^{(a)} \\
\left(1-\widetilde{\delta}_{+}\right) g_{\perp}^{(a)} & \longleftrightarrow & g_{\perp}^{(v)} \\
h_{\|}^{(t)} & \longleftrightarrow & h_{\|}^{(t)} \\
\left(1-\delta_{+}\right) h_{\|}^{(s)} & \longleftrightarrow & h_{\|}^{(p)}
\end{array}
$$

$\begin{array}{ccc}+\widetilde{\delta}_{ \pm} & \longleftrightarrow & -\widetilde{\delta}_{\mp} \\ +\delta_{ \pm} & \longleftrightarrow & -\delta_{\mp} \\ \mathcal{A} & \longleftrightarrow & \mathcal{V} \\ \mathcal{V} & \longleftrightarrow & \mathcal{A} \\ \mathcal{T} & \longleftrightarrow & \mathcal{T} \\ \Phi_{\|, \perp} & \longleftrightarrow & \Phi_{\|, \perp}\end{array}$

where the notations for LCDAs of vector mesons follow from Refs. [9, 10. For vector mesons, additional factors $\left(1-\widetilde{\delta}_{+}\right)$and $\left(1-\delta_{+}\right)$are considered [9] in the definitions of $g_{\perp}^{(a)}$ and $h_{\|}^{(s)}$, respectively, due to the normalizations $\int_{0}^{1} g_{\perp}^{(a)}(u) d u=\int_{0}^{1} h_{\|}^{(s)} d u=1$. Nevertheless, we do not need to put such factors in the definitions of axial-vector mesons; see results shown in Eqs. (261), (262), (263), (268), (269), and (270).

Table 1: The symmetric/antisymmetric properties of LCDAs under the interchange of the momentum fractions of the quark and antiquark in vector mesons and axial-vector mesons in the $\mathrm{SU}(3)$ limit, where the definitions of LCDAs of vector mesons follow from Refs. [9, 10].

\begin{tabular}{|c||c|c|c|}
\hline LCDA & Vector meson & Axial-vector meson $\left(1^{3} P_{1}\right)$ & Axial-vector meson $\left(1^{1} P_{1}\right)$ \\
\hline$\Phi_{\|}$ & symmetric & symmetric & antisymmetric \\
$\Phi_{\perp}$ & symmetric & antisymmetric & symmetric \\
$g_{\perp}^{(a)}$ & symmetric & symmetric & antisymmetric \\
$g_{\perp}^{(v)}$ & symmetric & symmetric & antisymmetric \\
$h_{\|}^{(t)}$ & symmetric & antisymmetric & symmetric \\
$h_{\|}^{(s)}$ or $h_{\|}^{(p)}$ & symmetric & antisymmetric & symmetric \\
$\mathcal{A}$ & symmetric & antisymmetric & symmetric \\
$\mathcal{V}$ & antisymmetric & symmetric & antisymmetric \\
$\mathcal{T}$ & antisymmetric & symmetric & antisymmetric \\
\hline
\end{tabular}




\section{Determinations of leading-twist LCDAs}

\subsection{Physical properties for axial-vector mesons}

In the quark model, the light $1^{1} P_{1}$ states, referred to as $b_{1}(1235), h_{1}(1170), h_{1}(1380)$, and $K_{1 B}$, form the $1^{+-}$nonets, whereas the light $1^{3} P_{1}$ mesons, denoted as $a_{1}(1260), f_{1}(1285)$, $f_{1}(1420)$, and $K_{1 A}$, form the $1^{++}$nonets. $h_{1}(1380)$ is not experimentally well-established [28] and its quark content was suggested as $\bar{s} s$ in the QCD sum rule calculation [19]. It should be noted that the real physical states $K_{1}(1270)$ and $K_{1}(1400)$ are the mixtures of $1^{3} P_{1}\left(K_{1 A}\right)$ and $1^{1} P_{1}\left(K_{1 B}\right)$ states; following the convention in Ref. [29], the relations can be written as

$$
\begin{aligned}
\left|K_{1}(1270)\right\rangle & =\left|K_{1 A}\right\rangle \sin \theta_{K}+\left|K_{1 B}\right\rangle \cos \theta_{K}, \\
\left|K_{1}(1400)\right\rangle & =\left|K_{1 A}\right\rangle \cos \theta_{K}-\left|K_{1 B}\right\rangle \sin \theta_{K} .
\end{aligned}
$$

In Ref. [29], two possible solutions with two-fold ambiguity $\left|\theta_{K}\right| \approx 33^{\circ}$ and $57^{\circ}$ were obtained. A similar constraint $35^{\circ} \lesssim\left|\theta_{K}\right| \lesssim 55^{\circ}$ was found in Ref. [30]. Therefore, the favor values may lie in the range $\left|\theta_{K}\right| \simeq(45 \pm 12)^{\circ}$. Just for simplicity, we will take $\theta_{K}=45^{\circ},-45^{\circ}$ as the reference points. Analogous to $\eta$ and $\eta^{\prime}$, for $1^{3} P_{1}$ states, $f_{1}(1285)$ and $f_{1}(1420)$ are mixed in terms of the pure octet $f_{8}$ and singlet $f_{1}$ due to $\mathrm{SU}(3)$ breaking effects, and can be parameterized as

$$
\left.\left.\mid f_{1}(1285)\right)\right\rangle=\left|f_{1}\right\rangle \cos \theta_{3} P_{1}+\left|f_{8}\right\rangle \sin \theta_{3} P_{1}, \quad\left|f_{1}(1420)\right\rangle=-\left|f_{1}\right\rangle \sin \theta_{3} P_{1}+\left|f_{8}\right\rangle \cos \theta_{3} P_{1} .
$$

From the Gell-Mann-Okubo mass formula [31, 28], it follows that

$$
\cos ^{2} \theta_{3} P_{1}=\frac{3 m_{f_{1}(1285)}^{2}-\left(4 m_{K_{1 A}}^{2}-m_{a_{1}}^{2}\right)}{3\left(m_{f_{1}(1285)}^{2}-m_{f_{1}(1420)}^{2}\right)},
$$

where

$$
m_{K_{1 A}}^{2}=m_{K_{1}(1400)}^{2} \cos ^{2} \theta_{K}+m_{K_{1}(1270)}^{2} \sin ^{2} \theta_{K} .
$$

Substituting into Eq. (74) with $\theta_{K}=(45 \pm 12)^{\circ}$, we then obtain $6 \theta_{3} P_{1}=38_{-16^{\circ}}^{\circ}$ which is consistent with the value of replacing $m^{2}$ by $m$ throughout Eq. (74). The previous phenomenological analyses suggested $\theta_{3} P_{1} \simeq 50^{\circ}$ [32]. In the present paper, we will take $\theta_{3} P_{1}=38^{\circ}$ or $50^{\circ}$ as the reference input for LCDA studies.

6 Since

$$
\tan \theta_{3 P_{1}}=\frac{4 m_{K_{1 A}}^{2}-m_{a_{1}}^{2}-3 m_{f_{1}(1420)}^{2}}{3 m_{18}^{2}}>0,
$$

where $m_{18}^{2}=\left\langle f_{1}|\mathcal{H}| f_{8}\right\rangle<0$ with $\mathcal{H}$ being the Hamiltonian, we find that $\theta_{3} P_{1}>0$. In the present paper, we can extend the study in the traditional quark model to a field-theoretical consideration that each Fock state of $f_{1}\left[\right.$ or $\left.f_{8}\right]$ is proportional to $(\bar{q} q)^{m} g^{n}(\bar{u} u+\bar{d} d+\bar{s} s) / \sqrt{3}\left[\right.$ or $\left.(\bar{q} q)^{m} g^{n}(\bar{u} u+\bar{d} d-2 \bar{s} s) / \sqrt{6}\right]$, where there is a relative sign difference between the $\bar{s} s$ contents of $f_{1}$ and $f_{8}$ in our convention. 
Similarly, for $1^{1} P_{1}$ states, $h_{1}(1170)$ and $h_{1}(1380)$ may be mixed in terms of the pure octet $h_{8}$ ad singlet $h_{1}$,

$$
\left.\left.\mid h_{1}(1170)\right)\right\rangle=\left|h_{1}\right\rangle \cos \theta_{1_{P_{1}}}+\left|h_{8}\right\rangle \sin \theta_{P_{1}}, \quad\left|h_{1}(1380)\right\rangle=-\left|h_{1}\right\rangle \sin \theta_{P_{1}}+\left|h_{8}\right\rangle \cos \theta_{{ }^{1} P_{1}} .
$$

Again from the Gell-Mann-Okubo mass formula, we obtain

$$
\cos ^{2} \theta_{1_{P_{1}}}=\frac{3 m_{h_{1}(1170)}^{2}-\left(4 m_{K_{1 B}}^{2}-m_{b_{1}}^{2}\right)}{3\left(m_{h_{1}(1170)}^{2}-m_{h_{1}(1380)}^{2}\right)},
$$

where

$$
m_{K_{1 B}}^{2}=m_{K_{1}(1400)}^{2} \sin ^{2} \theta_{K}+m_{K_{1}(1270)}^{2} \cos ^{2} \theta_{K} .
$$

We thus get $\theta_{P_{1}} \simeq 10_{-10^{\circ}}^{\circ}$ which coincides with the value of replacing $m^{2}$ by $m$ throughout Eq. (78). Note that $\left|\theta_{K}\right| \geq 50^{\circ}$ is disfavored because of the constraint $0 \leq \cos ^{2} \theta_{1} P_{1} \leq 1$. Note also that the QCD sum calculation suggested $\theta_{1_{1}} \simeq 45^{\circ}$, from which the predictive content of $h_{1}(1380)$ is predominated by the $\bar{s} s$ pain [19]. By comparing results with $\theta_{1} P_{1}=10^{\circ}$ and $45^{\circ}$ in the phenomenological LCDA analysis, we will show the effects induced by different mixing angles.

\subsection{Axial-vector couplings of $1^{3} P_{1}$ mesons}

In this subsection, we calculate renormalization-group (RG) improved QCD sum rules for the axial-vector couplings of $1^{3} P_{1}$ mesons. Because the QCD sum rule approach may not sufficiently determine the singlet-octet mixing angle, we thus calculate the QCD sum rules for pure $1^{3} P_{1}$ states.

\subsubsection{QCD sum rules for axial-vector couplings}

To evaluate the axial-vector couplings $f_{3} P_{1}$ for $1^{3} P_{1}$ mesons, we consider the two-point correlation function,

$$
\Pi_{\mu \nu}\left(q^{2}\right)=i \int d^{4} x e^{i q x}\langle 0| \mathrm{T}\left(j_{\mu}(x) j_{\nu}^{\dagger}(0)|0\rangle=-\Pi_{1}\left(q^{2}\right) g_{\mu \nu}+\Pi_{2}\left(q^{2}\right) q_{\mu} q_{\nu},\right.
$$

where the interpolating current $j_{\mu}=\bar{q}_{2} \gamma_{\mu} \gamma_{5} q_{1}$ satisfies

$$
\left\langle 0\left|j_{\mu}(0)\right| 1^{3} P_{1}(P, \lambda)\right\rangle=-i f_{{ }^{3} P_{1}} m_{3} P_{1} \epsilon_{\mu}^{(\lambda)} .
$$

In the massless quark limit, we have $\Pi_{1}=q^{2} \Pi_{2}$ due to conservation of $j_{\mu}$. Here we focus on $\Pi_{1}$ since $\Pi_{1}$ receives contributions only from axial-vector $\left({ }^{3} P_{1}\right)$ mesons, whereas $\Pi_{2}$ contains effects from pseudoscalar mesons. The above correlation function can be calculated from

\footnotetext{
${ }^{7}$ With the same reason as footnote $6 \theta_{1} P_{1}$ should be positive in sign.
} 
the hadron and quark-gluon dynamical points of view, respectively. The lowest-lying $1^{3} P_{1}$ meson contribution can be approximated via the dispersion relation as

$$
\frac{m_{3}^{2} P_{1} f_{3}^{2} P_{1}}{m_{3}^{2} P_{1}-q^{2}}=\frac{1}{\pi} \int_{0}^{s_{0}^{s_{0}} P_{1}} d s \frac{\operatorname{Im} \Pi_{1}^{\mathrm{OPE}}(s)}{s-q^{2}}
$$

where $\Pi_{1}^{\mathrm{OPE}}$, the QCD operator-product-expansion (OPE) result of $\Pi_{1}$ at the quark-gluon level, given in Ref. [19] up to dimension 6 and with $\mathcal{O}\left(\alpha_{s}\right)$ corrections, reads

$$
\begin{aligned}
\Pi_{1}^{\mathrm{OPE}}\left(q^{2}\right)= & -\frac{1}{4 \pi^{2}} q^{2} \ln \frac{-q^{2}}{\mu^{2}}\left(1+\frac{\alpha_{s}}{\pi}\right)+\frac{1}{q^{2}}\left(\frac{1}{12}\left\langle\frac{\alpha_{s}}{\pi} G^{2}\right\rangle-m_{2}\left\langle\bar{q}_{1} q_{1}\right\rangle-m_{1}\left\langle\bar{q}_{2} q_{2}\right\rangle\right) \\
& +\frac{1}{q^{4}}\left(\frac{2}{9} \pi \alpha_{s}\left\langle\left(\bar{q}_{1} \gamma_{\mu} \lambda^{a} q_{1}+\bar{q}_{2} \gamma_{\mu} \lambda^{a} q_{2}\right) \sum_{q} \bar{q} \gamma^{\mu} \lambda^{a} q\right\rangle+2 \pi \alpha_{s}\left\langle\bar{q}_{1} \lambda^{a} q_{2} \bar{q}_{2} \lambda^{a} q_{1}\right\rangle\right)
\end{aligned}
$$

and $s_{0}^{3} P_{1}$ is the threshold of the higher resonant states, such that the contributions of higher resonances are modeled by

$$
\frac{1}{\pi} \int_{s_{0}{ }^{3} P_{1}}^{\infty} d s \frac{\operatorname{Im} \Pi_{1}^{\mathrm{OPE}}(s)}{s-q^{2}}
$$

We further apply the Borel (inverse-Laplace) transformation to both sides of Eq. (82)

$$
\mathbf{B}\left[f\left(q^{2}\right)\right]=\lim _{\substack{n \rightarrow \infty \\-q^{2} \rightarrow \infty \\-q^{2} / n^{2}=M^{2} \text { fixed }}}\left(-q^{2}\right)^{n+1}\left[\frac{d}{d q^{2}}\right]^{n} f\left(q^{2}\right),
$$

to improve the convergence of the OPE series and further suppress the contributions from higher resonances. Moreover, we adopt the vacuum saturation approximation for describing the four-quark condensates in the present work, i.e.,

$$
\left\langle 0\left|\bar{q} \Gamma_{i} \lambda^{a} q \bar{q} \Gamma_{i} \lambda^{a} q\right| 0\right\rangle=-\frac{1}{16 N_{c}^{2}} \operatorname{Tr}\left(\Gamma_{i} \Gamma_{i}\right) \operatorname{Tr}\left(\lambda^{a} \lambda^{a}\right)\langle\bar{q} q\rangle^{2},
$$

and neglect the possible corrections due to their anomalous dimensions. Finally, we arrive at the $f_{3} P_{1}$ sum rules, given by

$$
\begin{aligned}
f_{{ }^{2} P_{1}}^{2} m_{3}^{2}{ }_{P_{1}} e^{-m_{3_{1}}^{2} / M^{2}}= & \frac{1}{4 \pi^{2}} \int_{0}^{s_{0} P_{P_{1}}} s d s e^{-s / M^{2}}\left(1+\frac{\alpha_{s}}{\pi}\right)-\frac{1}{12}\left\langle\frac{\alpha_{s}}{\pi} G^{2}\right\rangle+m_{q_{2}}\left\langle\bar{q}_{1} q_{1}\right\rangle+m_{q_{1}}\left\langle\bar{q}_{2} q_{2}\right\rangle \\
& -\frac{1}{M^{2}}\left[\frac{32 \pi \alpha_{s}}{81}\left(\left\langle\bar{q}_{2} q_{2}\right\rangle^{2}+\left\langle\bar{q}_{1} q_{1}\right\rangle^{2}\right)+\frac{32 \pi \alpha_{s}}{9}\left\langle\bar{q}_{2} q_{2}\right\rangle\left\langle\bar{q}_{1} q_{1}\right\rangle\right] .
\end{aligned}
$$




\subsubsection{Results for $1^{3} P_{1}$ mesons}

We start with the analysis of the $f_{3} P_{1}$ sum rules. To examine the quality of the sum rules, we also give the mass sum rule results. The mass sum rule for the $1^{3} P_{1}$ lowest-lying resonance can be obtained by taking the logarithm of both sides of Eq. (87) and then applying the differential operator $M^{4} \partial / \partial M^{2}$ to them, where $s_{0}^{3} P_{1}$ is determined by the maximum stability of the sum rules. Substituting the obtained $s_{0}^{3} P_{1}$ and masses into Eq. (87), one arrives at the sum rules for the decay constants $f_{3} P_{1}$. In the numerical analysis, we use the parameters which are given in Appendix $\mathrm{C}$ and choose the Borel window $0.8 \mathrm{GeV}^{2}<M^{2}<1.3 \mathrm{GeV}^{2}$, where the contribution originating from higher resonances (and the continuum) is less than $44 \%$ and the highest OPE term at the quark level is no more than $14 \%$. Note that in the sum rules, the contributions from higher resonances are modeled by

$$
\frac{1}{\pi} \int_{s_{0}}^{\infty} d s e^{-s / M^{2}} \operatorname{Im} \Pi_{1}^{\mathrm{OPE}}(s) .
$$

In Fig. 1, the masses and decay constants are plotted as functions of the Borel mass squared $M^{2}$. The results are summarized in Table 2. Introducing the decay constants $f_{f_{1}(1285)}^{q}$ and $f_{f_{1}(1420)}^{q}$ by

$$
\begin{aligned}
& \left\langle 0\left|\bar{q} \gamma_{\mu} \gamma_{5} q\right| f_{1}(1285)(P, \lambda)\right\rangle=-i m_{f_{1}(1285)} f_{f_{1}(1285)}^{q} \epsilon_{\mu}^{(\lambda)} \\
& \left\langle 0\left|\bar{q} \gamma_{\mu} \gamma_{5} q\right| f_{1}(1420)(P, \lambda)\right\rangle=-i m_{f_{1}(1420)} f_{f_{1}(1420)}^{q} \epsilon_{\mu}^{(\lambda)}
\end{aligned}
$$

we get

$$
\begin{aligned}
f_{f_{1}(1285)}^{u} & =\frac{f_{f_{1}}}{\sqrt{3}} \frac{m_{f_{1}}}{m_{f_{1}(1285)}} \cos \theta_{3} P_{1} \\
f_{f_{1}(1285)}^{s} & =\frac{f_{f_{1}}}{\sqrt{3}} \frac{m_{f_{1}}}{\sqrt{6}} \frac{m_{f_{8}}}{m_{f_{1}(1285)}} \sin \theta^{3} P_{1}=173 \pm 23(167 \pm 22) \mathrm{MeV} \\
f_{f_{1}(1420)}^{u} & =-\frac{f_{f_{1}}}{\sqrt{3}} \frac{m_{f_{1}}}{m_{f_{1}(1420)}} \sin \theta_{3} P_{1}+\frac{2 f_{f_{8}}}{\sqrt{6}} \frac{m_{f_{8}}}{m_{f_{1}(1285)}} \sin \theta_{3} P_{1}=-9 \pm 13(-59 \pm 18) \mathrm{MeV} \\
f_{f_{8}} & \frac{m_{f_{8}}}{m_{f_{1}(1420)}} \cos \theta^{3} P_{1}=-9 \pm 10(-41 \pm 11) \mathrm{MeV},(93) \\
& =-\frac{f_{f_{1}}}{\sqrt{3}} \frac{m_{f_{1}}}{m_{f_{1}(1420)}} \sin \theta_{3} P_{1}-\frac{2 f_{f_{8}}}{\sqrt{6}} \frac{m_{f_{8}}}{m_{f_{1}(1420)}} \cos \theta^{3} P_{1} \\
& =-217 \pm 27(-211 \pm 26) \mathrm{MeV},
\end{aligned}
$$

corresponding to $\theta^{3} P_{1}=38^{\circ}\left(50^{\circ}\right)$. In particular, $f_{1}(1285)$ and $f_{1}(1420)$ are predominated by their $\bar{u} u$ and $\bar{s} s$ contents, respectively.

Using Eq. (72), the decay constants for $K_{1(1270)}$ and $K_{1(1400)}$ read (with $\bar{q}=\bar{u}$ or $\bar{d}$ )

$$
\begin{aligned}
\left\langle 0\left|\bar{q} \gamma_{\mu} \gamma_{5} s\right| K_{1}(1270)(P, \lambda)\right\rangle & =-i f_{K_{1}(1270)} m_{K_{1}(1270)} \epsilon_{\mu}^{(\lambda)} \\
& =-i\left(f_{K_{1 A}} m_{K_{1 A}} \sin \theta_{K}+f_{K_{1 B}} m_{K_{1 B}} a_{0}^{\|, K_{1 B}} \cos \theta_{K}\right) \epsilon_{\mu}^{(\lambda)}
\end{aligned}
$$


Table 2: The sum rule results of masses, decay constants, and corresponding excited thresholds $s_{0}^{3} P_{1}$ for $1^{3} P_{1}$ mesons.

\begin{tabular}{|c|ccc|}
\hline State & Mass $[\mathrm{GeV}]$ & Decay cosntant $f_{P_{1}}[\mathrm{MeV}]$ & $s_{0}{ }^{3}{ }_{1}\left[\mathrm{GeV}^{2}\right]$ \\
\hline$a_{1}(1260)$ & $1.23 \pm 0.06$ & $238 \pm 10$ & $2.55 \pm 0.15$ \\
$f_{1}\left(1^{3} P_{1}\right)$ & $1.28 \pm 0.06$ & $245 \pm 13$ & $2.80 \pm 0.20$ \\
$f_{8}\left(1^{3} P_{1}\right)$ & $1.29 \pm 0.05$ & $239 \pm 13$ & $2.70 \pm 0.20$ \\
$K_{1 A}$ & $1.31 \pm 0.06$ & $250 \pm 13$ & $2.90 \pm 0.20$ \\
\hline
\end{tabular}

and

$$
\begin{aligned}
\left\langle 0\left|\bar{q} \gamma_{\mu} \gamma_{5} s\right| K_{1}(1400)(P, \lambda)\right\rangle & =-i f_{K_{1}(1400)} m_{K_{1}(1400)} \epsilon_{\mu}^{(\lambda)} \\
& =-i\left(f_{K_{1 A}} m_{K_{1 A}} \cos \theta_{K}-f_{K_{1 B}} m_{K_{1 B}} a_{0}^{\|, K_{1 B}} \sin \theta_{K}\right) \epsilon_{\mu}^{(\lambda)}
\end{aligned}
$$

where $f_{K_{1 A}}$ and $m_{K_{1 A}}$ are given in Table2, and $a_{0}^{\|, K_{1 B}}$ (with $f_{K_{1 B}}=f_{K_{1 B}}^{\perp}(1 \mathrm{GeV}$ ) by definition in this paper) and $m_{K_{1 B}}$ can be found in Tables 3 and 4 , respectively. (See the detailed discussion in Sec. 4.3.) Numerically, we thus obtain

$$
\begin{aligned}
& f_{K_{1}(1270)}=197 \pm 15 \mathrm{MeV}, \\
& f_{K_{1}(1400)}=151 \pm 12 \mathrm{MeV}, \quad \text { if taking } \theta_{K}=45^{\circ}
\end{aligned}
$$

and

$$
\begin{aligned}
& f_{K_{1}(1270)}=-166 \pm 11 \mathrm{MeV}, \\
& f_{K_{1}(1400)}=179 \pm 12 \mathrm{MeV}, \quad \text { if taking } \theta_{K}=-45^{\circ}
\end{aligned}
$$

where the correlation of the errors between the masses and decay constants is considered. It is interesting to note that, if one sets $a_{0}^{\|, K_{1 B}}=0$, then the central value of $f_{K_{1}(1270)}$ becomes $\pm 182 \mathrm{GeV}$ corresponding to $\theta_{K}= \pm 45^{\circ}$, whereas that of $f_{K_{1}(1400)}$ is $165 \mathrm{GeV}$ independent of the sign of $\theta_{K}$; the local axial-vector current can couple only to $K_{1 A}$, but not to $K_{1 B}$ in the $\mathrm{SU}(3)$ limit.

\subsection{Tensor couplings of $1^{1} P_{1}$ mesons}

The tensor couplings of $1^{1} P_{1}$ mesons are defined as

$$
\left\langle 0\left|\bar{q}_{2} \sigma_{\mu \nu} q_{1}\right| 1^{1} P_{1}(P, \lambda)\right\rangle=i f_{{ }^{1} P_{1}}^{\perp} \epsilon_{\mu \nu \alpha \beta} \epsilon_{(\lambda)}^{\alpha} P^{\beta}
$$



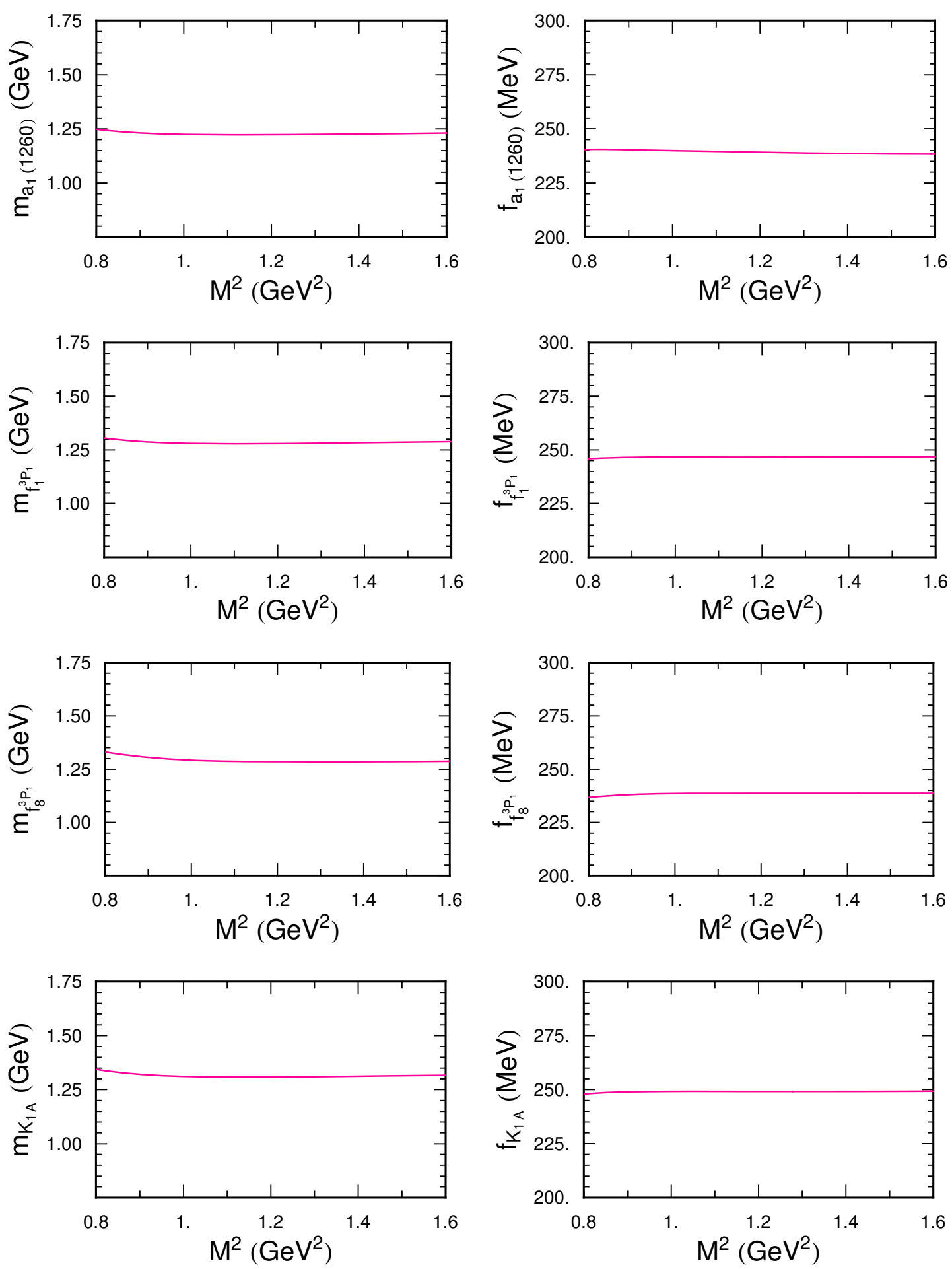

Figure 1: masses and decay constants of the $1^{3} P_{1}$ states as functions of the Borel mass squared, where the central values of input parameters given in Appendix C have been used. 
i.e.,

$$
\left\langle 0\left|\bar{q}_{2} \sigma^{\mu \nu} \gamma_{5} q_{1}\right| 1^{1} P_{1}(P, \lambda)\right\rangle=-f_{{ }_{1} P_{1}}^{\perp}\left(\epsilon_{(\lambda)}^{\mu} P^{\nu}-\epsilon_{(\lambda)}^{\nu} P^{\mu}\right) .
$$

In Ref. [18, we have calculated RG-improved QCD sum rules of the tensor couplings for $1^{1} P_{1}$ mesons, where we assumed that $h_{1}(1170)$ is the same as $b_{1}(1235)$, while $h_{1}(1380)$ is made of $\bar{s} s$. Here we instead examine the pure $1^{1} P_{1}$ octet states. The results are collected in Table 3. We have estimated the singlet-octet mixing angle by means of Gell-Mann-Okubo mass formula. Thus, the decay constants for the real states $h_{1}(1170)$ and $h_{1}(1380)$ can be obtained. See the results listed in Eqs. (105)-(108). We have also updated the values for $b_{1}(1235)$ and and $K_{1 B}$ states due to the following two more reasons. First, we take into account the uncertainties of the condensates and quark mass which were not fully considered in Ref. [16]. Second, we re-examine the stability of the sum rules and choose to use the Borel window $0.8 \mathrm{GeV}^{2}<M^{2}<1.3 \mathrm{GeV}^{2}$ in the analysis. During this window, which covers the plateau region for the masses and decay constants versus $M^{2}$, the contributions from excited states (including the continuum) and from the highest dimension term in OPE expansion are less than $43 \%$ and $5 \%$, respectively, while for the previous choice with the upper bound $1.5 \mathrm{GeV}^{2}$ the contributions of excited states can reach $52 \%$. Although the region for the present Borel window is a little lower than the previous study in Ref. [18], the present results should be more reliable. Note that it will become questionable for the sum rule results if one further reduces the lower bound of the Borel window. The reason is because in the lower Borel mass region the effects owing to the uncertainty of the highest dimensions term, for which we have assumed the vacuum saturation approximation, and radiative corrections become much more important and are out of control.

Since the QCD sum rule approach may not sufficiently determine the singlet-octet mixing angle, we thus study the QCD sum rules for pure $1^{1} P_{1}$ states; in other words, we neglect the corrections arising from $\left\langle h_{1}|\mathcal{H}| h_{8}\right\rangle$ due to $\left\langle h_{1}|\mathcal{H}| h_{8}\right\rangle \ll\left\langle h_{1}|\mathcal{H}| h_{1}\right\rangle,\left\langle h_{8}|\mathcal{H}| h_{8}\right\rangle$, where $\mathcal{H}$ is the Hamiltonian, in the studies of the mass and tensor coupling sum rules for $h_{1}, h_{8}$ and $K_{1 B}$ states. Employing the formulas given in Ref. [18], we obtain the results shown in Table 3 . In complete analogy to the discussion for $1^{3} P_{1}$ states, we introduce

$$
\begin{aligned}
& \left\langle 0\left|\bar{q} \sigma_{\mu \nu} q\right| h_{1}(1170)(P, \lambda)\right\rangle=i f_{h_{1}(1170)}^{\perp, q} \epsilon_{\mu \nu \alpha \beta} \epsilon_{(\lambda)}^{\alpha} P^{\beta}, \\
& \left\langle 0\left|\bar{q} \sigma_{\mu \nu} q\right| h_{1}(1380)(P, \lambda)\right\rangle=i f_{h_{1}(1380)}^{\perp, q} \epsilon_{\mu \nu \alpha \beta} \epsilon_{(\lambda)}^{\alpha} P^{\beta},
\end{aligned}
$$

and then obtain

$$
\begin{aligned}
& f_{h_{1}(1170)}^{\perp, u}=\frac{f_{h_{1}}}{\sqrt{3}} \cos \theta_{1_{P_{1}}}+\frac{f_{h_{8}}}{\sqrt{6}} \sin \theta_{1_{P_{1}}}=116 \pm 8(128 \pm 7) \mathrm{MeV}, \\
& f_{h_{1}(1170)}^{\perp, s}=\frac{f_{h_{1}}}{\sqrt{3}} \cos \theta_{1_{1}}-\frac{2 f_{h_{8}}}{\sqrt{6}} \sin \theta_{P_{1}}=75 \pm 8(-36 \pm 10) \mathrm{MeV}, \\
& f_{h_{1}(1380)}^{\perp, u}=-\frac{f_{h_{1}}}{\sqrt{3}} \sin \theta_{1_{P_{1}}}+\frac{f_{h_{8}}}{\sqrt{6}} \cos \theta_{1_{P_{1}}}=58 \pm 5(-19 \pm 7) \mathrm{MeV},
\end{aligned}
$$




$$
f_{h_{1}(1380)}^{\perp, s}=-\frac{f_{h_{1}}}{\sqrt{3}} \sin \theta_{1_{P_{1}}}-\frac{2 f_{h_{8}}}{\sqrt{6}} \cos \theta_{1_{P_{1}}}=-171 \pm 9(-183 \pm 10) \mathrm{MeV},
$$

corresponding to $\theta_{P_{1}}=10^{\circ}\left(45^{\circ}\right)$. As $\theta_{1_{1}}=45^{\circ}, h_{1}(1380)$ is therefore dominated by the $\bar{s} s$ content, and $h_{1}(1170)$ can be approximated by $(\bar{u} u+\bar{d} d) / \sqrt{2}$. However, the $\bar{s} s$ content of $h_{1}(1170)$ and $\bar{u} u$ content of $h_{1}(1380)$ become significant for $\theta_{P_{1}}=10^{\circ}$.

As for strange mesons, we have (with $\bar{q} \equiv \bar{u}, \bar{d}$ )

$$
\begin{aligned}
\left\langle 0\left|\bar{q} \sigma_{\mu \nu} s\right| K_{1}(1270)(P, \lambda)\right\rangle & =i f_{K_{1}(1270)}^{\perp} \epsilon_{\mu \nu \alpha \beta} \epsilon_{(\lambda)}^{\alpha} P^{\beta} \\
& =i\left(f_{K_{1 A}}^{\perp} a_{0}^{\perp, K_{1 A}} \sin \theta_{K}+f_{K_{1 B}}^{\perp} \cos \theta_{K}\right) \epsilon_{\mu \nu \alpha \beta} \epsilon_{(\lambda)}^{\alpha} P^{\beta}
\end{aligned}
$$

and

$$
\begin{aligned}
\left\langle 0\left|\bar{q} \sigma_{\mu \nu} s\right| K_{1}(1400)(P, \lambda)\right\rangle & =i f_{K_{1}(1400)}^{\perp} \epsilon_{\mu \nu \alpha \beta} \epsilon_{(\lambda)}^{\alpha} P^{\beta} \\
& =i\left(f_{K_{1 A}}^{\perp} a_{0}^{\perp, K_{1 A}} \cos \theta_{K}-f_{K_{1 B}}^{\perp} \sin \theta_{K}\right) \epsilon_{\mu \nu \alpha \beta} \epsilon_{(\lambda)}^{\alpha} P^{\beta}
\end{aligned}
$$

where $f_{K_{1 B}}^{\perp}$ and $a_{0}^{\perp, K_{1 A}}$ are given in Tables 3 and 4, respectively, and use of $f_{K_{1 A}}^{\perp}=f_{K_{1 A}}=$ $(250 \pm 20) \mathrm{MeV}$ is made in the following by definition. Consequently, we obtain (at the scale $\mu=1 \mathrm{GeV})$

$$
\begin{aligned}
& f_{K_{1}(1270)}^{\perp}=145 \pm 15 \mathrm{MeV}, \\
& f_{K_{1}(1400)}^{\perp}=-124 \pm 15 \mathrm{MeV}, \quad \text { if taking } \theta_{K}=45^{\circ},
\end{aligned}
$$

and

$$
\begin{aligned}
& f_{K_{1}(1270)}^{\perp}=124 \pm 14 \mathrm{MeV}, \\
& f_{K_{1}(1400)}^{\perp}=145 \pm 14 \mathrm{MeV}, \quad \text { if taking } \theta_{K}=-45^{\circ},
\end{aligned}
$$

where $a_{0}^{\perp, K_{1 A}}$ gives about $8 \%$ corrections to the decay constants. If setting $a_{0}^{\perp, K_{1 A}}=0$, all the magnitudes of the central values of the decay constants are equal to $\sim 134 \mathrm{GeV}$.

\subsection{Gegenbauer moments of leading-twist LCDAs}

The Gegenbauer moments can be calculated from the standard QCD sum rule approach by adopting a relevant two-point correlation function as the starting point. It is interesting to note that in $\mathrm{SU}(3)$ symmetry limit the decay constant for a $1^{1} P_{1}$ state transiting to the vacuum via the local axial-vector current vanishes due to G-parity mismatch between the current and states. On the other hand, conventionally, the decay constants $f_{3} P_{1}$ for the local axial-vector currents coupling to the $1^{3} P_{1}$ states are chosen to be positive as in the present paper. Although the diagonal correlation functions may have good qualities for sum rule results of G-parity invariant parameters, it cannot determine the relative sign of the Gegenbauer moments. Moreover, the diagonal correlation functions cannot use to 
Table 3: The sum rule results of masses, decay constants, and corresponding excited thresholds $s_{0}^{1} P_{1}$ for $1^{1} P_{1}$ mesons. The values of $f_{{ }^{\perp} P_{1}}^{\perp}$ are given at the scale $\mu=1 \mathrm{GeV}$.

\begin{tabular}{|c|ccc|}
\hline State & Mass $[\mathrm{GeV}]$ & Decay cosntant $f_{{ }_{P_{1}}^{\perp}}[\mathrm{MeV}]$ & $s_{0}^{1} P_{1}\left[\mathrm{GeV}^{2}\right]$ \\
\hline$b_{1}(1235)$ & $1.21 \pm 0.07$ & $180 \pm 8$ & $2.6 \pm 0.2$ \\
$h_{1}\left(1^{1} P_{1}\right)$ & $1.23 \pm 0.07$ & $180 \pm 12$ & $2.6 \pm 0.2$ \\
$h_{8}\left(1^{1} P_{1}\right)$ & $1.37 \pm 0.07$ & $190 \pm 10$ & $3.2 \pm 0.2$ \\
$K_{1 B}$ & $1.34 \pm 0.08$ & $190 \pm 10$ & $3.1 \pm 0.2$ \\
\hline
\end{tabular}

evaluate the G-parity violating parameters due to the mixing between ${ }^{3} P_{1}$ and ${ }^{1} P_{1}$ states. To determine not only the magnitudes but also the relative signs for Gegenbauer moments relevant to the leading-twist LCDAs of $1^{3} P_{1}$ states, we thus choose one of the interpolating currents in the two-point correlation function to be the local axial-vector current $\bar{q}_{2} \gamma_{\mu} \gamma_{5} q_{1}$, i.e., we consider the non-diagonal correlation functions here. Note that we define the sum rule to be diagonal here only if two interpolating currents in the correlation function are exactly the same, but non-diagonal otherwise (See footnote 9 for further discussions). With the same reason, in the following section we also adopt the local axial-vector current as one of the interpolating currents in QCD sum rule studies to determine the relevant parameters for twist-3 three-parton LCDAs of $1^{3} P_{1}$ states, whereas we use the local pseudo-tensor current as one of the interpolating currents since the resulting contributions arising from ${ }^{3} P_{1}$ states vanish in $\mathrm{SU}(3)$ limit.

However, as we consider the non-diagonal correlation functions, G-parity breaking contributions of ${ }^{3} P_{1}$ and ${ }^{1} P_{1}$ states always mix. To obtain the G-parity violating Gegenbauer moments for LCDAs of ${ }^{3} P_{1}$ and ${ }^{1} P_{1}$ mesons, we will assume an additional reasonable constraint on the parameters (see Eq. (143)) ).

\subsubsection{Gegenbauer moments of $\Phi_{\|}^{3} P_{1}$ for $1^{3} P_{1}$ mesons}

The LCDAs $\Phi_{\|}^{3} P_{1}(u, \mu)$ corresponding to the $1^{3} P_{1}$ states, which are denoted by the superscript ${ }^{3} P_{1}$, with the quark contents $\bar{q}_{2}$ and $q_{1}$, are defined as

$$
\left\langle 1^{3} P_{1}(P, \lambda)\left|\bar{q}_{1}(y) \not z \gamma_{5} q_{2}(x)\right| 0\right\rangle=i f_{{ }^{3} P_{1}} m_{3} P_{1}\left(\epsilon^{*(\lambda)} \bar{z}\right) \int_{0}^{1} d x e^{i(u p \cdot y+\bar{u} p \cdot x)} \Phi_{\|}^{3 P_{1}}(u, \mu),
$$

where $u$ (or $\bar{u}=1-u$ ) is the momentum fraction carried by the quark $q_{1}$ (or antiquark $\bar{q}_{2}$ ) and $\mu$ is the renormalization scale of the LCDAs, and we have considered here and below that $\bar{z} \propto y-x, \bar{z}^{2}=0,(y-x)^{2}=0$, but $\bar{z} \not \rightarrow 0$ even for $y \rightarrow x . \Phi_{\|}^{3 P_{1}}(u, \mu)$ can be expanded in a series of Gegenbauer polynomials as given in Eq. (48). To evaluate the Gegenbauer 
moments of $\Phi_{\|}^{3} P_{1}$, we take into account the following two-point correlation function

$$
\Pi_{\mu}^{(l)}(q)=i \int d^{4} x e^{i q x}\langle 0| T\left(\Omega_{3 P_{1}}^{(l)}(x) O_{\mu}^{\dagger}(0)|0\rangle=(\bar{z} q)^{l}\left[-I_{1}^{(l)}\left(q^{2}\right) \bar{z}_{\mu}+I_{2}^{(l)}\left(q^{2}\right)(\bar{z} q) q_{\mu}\right]\right.
$$

where

$$
O_{\mu}(0)=\bar{q}_{2}(0) \gamma_{\mu} \gamma_{5} q_{1}(0)
$$

and the relevant multiplicatively renormalizable operator, to leading logarithmic (LO) accuracy, is

$$
\Omega_{3}^{(l)}(x)=\sum_{j=0}^{l} c_{l, j}(i \bar{z} \partial)^{l-j} \bar{q}_{2}(x) \not z \gamma_{5}(i \bar{z} \stackrel{\leftrightarrow}{D})^{j} q_{1}(x)
$$

with $\stackrel{\leftrightarrow}{D}_{\mu}=\vec{D}_{\mu}-\overleftarrow{D}_{\mu}=\left(\vec{\partial}+i g_{s} A^{a}(x) \lambda^{a} / 2\right)_{\mu}-\left(\overleftarrow{\partial}-i g_{s} A^{a}(x) \lambda^{a} / 2\right)_{\mu}$ and $c_{l, k}$ being the coefficients of the Gegenbauer polynomials such that $C_{l}^{3 / 2}(x)=\sum_{k=0}^{k=l} c_{l, k} x^{k} . \Omega_{3}^{(l)}$ and $O_{\mu}$ satisfy the following relations:

$$
\begin{aligned}
\left\langle 0\left|\Omega_{3 P_{1}}^{(l)}(0)\right| 1^{3} P_{1}(P, \lambda)\right\rangle & =-i f_{3} P_{1} m^{3} P_{1}\left(\epsilon^{(\lambda)} \bar{z}\right)(P \bar{z})^{l} \frac{3(l+1)(l+2)}{2(2 l+3)} a_{l}^{\|,{ }^{3} P_{1}}(\mu), \\
\left\langle 0\left|O_{\mu}(0)\right| 1^{3} P_{1}(P, \lambda)\right\rangle & =-i f_{3} P_{1} m_{3} P_{1} \epsilon_{\mu}^{(\lambda)} .
\end{aligned}
$$

We are interested only in $I_{1}^{(l)}\left(q^{2}\right)$ since only states with quantum numbers of ${ }^{3} P_{1}$ contribute to $I_{1}^{(l)}\left(q^{2}\right)$, whereas $I_{2}^{(l)}\left(q^{2}\right)$ receives contributions from states with quantum numbers of pseudoscalar mesons and ${ }^{3} P_{1} . I_{2}^{(l)}\left(q^{2}\right)$ was already given in Ref. 33. In the massless quark limit, one has $I_{1}^{(l)}=q^{2} I_{2}^{(l)}$. Nevertheless, the above relation is broken even for $m_{q_{1}}=m_{q_{2}} \neq 0$. The OPE result of $I_{1}^{(l)}$, up to dimension 6 and with $\mathcal{O}\left(\alpha_{s}\right)$ corrections, is given by (for $l \geq 1$ )

$$
\begin{aligned}
I_{1}^{(l) \mathrm{OPE}}= & -\frac{\alpha_{s}}{2 \pi^{3}} q^{2} \ln \frac{-q^{2}}{\mu^{2}} \int_{0}^{1} d u u \bar{u} C_{l}^{3 / 2}(2 u-1) \ln ^{2} \frac{u}{\bar{u}} \\
& -\frac{C_{l}^{3 / 2}(1)}{q^{2}}\left[m_{q_{1}}\left\langle\bar{q}_{2} q_{2}\right\rangle+m_{q_{2}}\left\langle\bar{q}_{1} q_{1}\right\rangle(-1)^{l}\right]+\frac{C_{l}^{3 / 2}(1)}{24 q^{2}}\left\langle\frac{\alpha_{s}}{\pi} G^{2}\right\rangle\left[1+(-1)^{l}\right] \\
& +\frac{2}{q^{4}} C_{l-1}^{5 / 2}(1) \theta(l-1)\left[m_{q_{2}}\left\langle\bar{q}_{1} g_{s} \sigma G q_{1}\right\rangle(-1)^{l}+m_{q_{1}}\left\langle\bar{q}_{2} g_{s} \sigma G q_{2}\right\rangle\right] L^{-14 /(3 b)} \\
& -\frac{16 \pi \alpha_{s}}{9 q^{4}} C_{l}^{3 / 2}(1)\left\langle\bar{q}_{1} q_{1}\right\rangle\left\langle\bar{q}_{2} q_{2}\right\rangle\left[1+(-1)^{l}\right] \\
& -\frac{32 \pi \alpha_{s}}{81 q^{4}}\left[C_{l}^{3 / 2}(1)\right]^{2}\left[\left\langle\bar{q}_{1} q_{1}\right\rangle^{2}(-1)^{l}+\left\langle\bar{q}_{2} q_{2}\right\rangle^{2}\right]
\end{aligned}
$$

where the terms containing quark mass corrections and the results with odd $l$ are new. For even $l$ and in the massless quark limit, the above result for $I_{1}^{(l) \mathrm{OPE}}$ is consistent with $q^{2} I_{2}^{(l) \mathrm{OPE}}$ given in Ref. [33]. 
The quality of the sum rules obtained directly from $I_{1}^{(l) \mathrm{OPE}}$ is not good. See the discussion below. Another way to obtain the sum rules is to take into account the dispersion relation with one subtraction. This method was introduced in Refs. [34, 35]. Consider $\tilde{I}_{1}^{(l)}\left(q^{2}\right)=$ $I_{1}^{(l)}\left(q^{2}\right)-I_{1}^{(l), \text { pert }}\left(q^{2}\right) . \tilde{I}_{1}^{(l)}\left(q^{2}\right)$ is finite in $-q^{2} \rightarrow \infty$, and therefore we can write down

$$
\begin{aligned}
\tilde{I}_{1}^{(l)}\left(q^{2}\right) & =\tilde{I}_{1}^{(l)}(0)-\frac{-q^{2}}{\pi} \int_{0}^{\infty} \frac{d s}{s\left(s-q^{2}\right)}\left[\rho_{\text {phys }}(s)-\operatorname{Im} I_{1}^{(l), p e r t}(s)\right], \\
& =\tilde{I}_{1}^{(l)}(0)-\frac{-q^{2}}{\pi} \int_{0}^{\infty} \frac{d s}{s\left(s-q^{2}\right)}\left[\rho_{1^{3} P_{1}}(s)-\theta\left(s_{0}^{\|}-s\right) \operatorname{Im} I_{1}^{(l), p e r t}(s)\right],
\end{aligned}
$$

where $\rho_{\text {phys }}$ and $\rho_{1^{3} P_{1}}$ are the total physical spectral density and the lowest-lying $\left(1^{3} P_{1}\right)$ spectral density 8 , respectively, and where we have modeled the higher resonance states as

$$
\rho_{\text {phys }}(s)=\rho_{1^{3} P_{1}}(s)+\theta\left(s-s_{0}^{\|}\right) \operatorname{Im} I_{1}^{(l), \text { pert }}(s) .
$$

On the other hand, one should note that ${ }^{1} P_{1}$ states can still enter the sum results by the G-parity violating effect, which is due to $m_{q_{1}}-m_{q_{2}} \neq 0$,

$$
\left\langle 1^{1} P_{1}(P, \lambda)\left|\bar{q}_{1}(0) \gamma_{\mu} \gamma_{5} q_{2}(0)\right| 0\right\rangle=i f_{{ }^{1} P_{1}} a_{0}^{\|,{ }^{1} P_{1}} m_{1} P_{1} \epsilon_{\mu}^{*(\lambda)} .
$$

If one derives the Gegenbauer moment sum rules directly from Eq. (121), then the results are

$$
\begin{aligned}
& {\left[a_{l}^{\|,{ }^{3} P_{1}} m_{{ }^{2} P_{1}}^{2} f_{{ }^{2} P_{1}}^{2} e^{-m_{3_{P}}^{2} / M^{2}}+a_{l}^{\|,{ }^{1} P_{1}} a_{0}^{\|,{ }^{1} P_{1}} m_{{ }^{2}}^{2} f_{1} f_{P_{1}}^{2} e^{-m_{1}^{2}{ }_{P_{1}} / M^{2}}\right] \frac{3(l+1)(l+2)}{2(2 l+3)} L^{-\gamma_{(l)}^{\|} / b}} \\
& =\frac{\alpha_{s}}{2 \pi^{3}} M^{4}\left[1-e^{-s_{0}^{\|} / M^{2}}\left(1+\frac{s_{0}^{\|,{ }^{3} P_{1}}}{M^{2}}\right)\right] \int_{0}^{1} d u u \bar{u} C_{l}^{3 / 2}(2 u-1) \ln ^{2} \frac{u}{\bar{u}} \\
& \quad+C_{l}^{3 / 2}(1)\left(m_{q_{1}}\left\langle\bar{q}_{2} q_{2}\right\rangle+m_{q_{2}}\left\langle\bar{q}_{1} q_{1}\right\rangle\right)-\frac{C_{l}^{3 / 2}(1)}{12}\left\langle\frac{\alpha_{s}}{\pi} G^{2}\right\rangle \\
& \quad+\frac{2}{M^{2}} C_{l-1}^{5 / 2}(1) \theta(l-1)\left(m_{q_{1}}\left\langle\bar{q}_{2} g_{s} \sigma G q_{2}\right\rangle+m_{q_{2}}\left\langle\bar{q}_{1} g_{s} \sigma G q_{1}\right\rangle\right) L^{-14 /(3 b)} \\
& \quad-\frac{32 \pi \alpha_{s}}{9 M^{2}} C_{l}^{3 / 2}(1)\left\langle\bar{q}_{1} q_{1}\right\rangle\left\langle\bar{q}_{2} q_{2}\right\rangle-\frac{32 \pi \alpha_{s}}{81 M^{2}}\left[C_{l}^{3 / 2}(1)\right]^{2}\left(\left\langle\bar{q}_{1} q_{1}\right\rangle^{2}+\left\langle\bar{q}_{2} q_{2}\right\rangle^{2}\right)
\end{aligned}
$$

for even $l \geq 2$ and

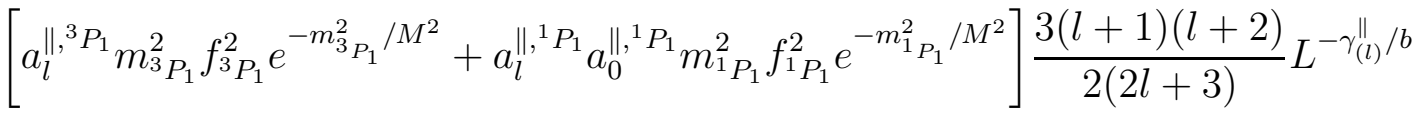

$$
\begin{aligned}
& =C_{l}^{3 / 2}(1)\left(m_{q_{1}}\left\langle\bar{q}_{2} q_{2}\right\rangle-m_{q_{2}}\left\langle\bar{q}_{1} q_{1}\right\rangle\right) \\
& +\frac{2}{M^{2}} C_{l-1}^{5 / 2}(1) \theta(l-1)\left(m_{q_{1}}\left\langle\bar{q}_{2} g_{s} \sigma G q_{2}\right\rangle-m_{q_{2}}\left\langle\bar{q}_{1} g_{s} \sigma G q_{1}\right\rangle\right) L^{-14 /(3 b)} \\
& +\frac{32 \pi \alpha_{s}}{81 M^{2}}\left[C_{l}^{3 / 2}(1)\right]^{2}\left(\left\langle\bar{q}_{1} q_{1}\right\rangle^{2}-\left\langle\bar{q}_{2} q_{2}\right\rangle^{2}\right)
\end{aligned}
$$

\footnotetext{
${ }^{8}$ Here we also need to consider $1^{1} P_{1}$ state which will contribute to the sum rules due to the G-parity violating effect. See Eq. (124).
} 
for odd $l$ (of the $K_{1 A}$ meson), where $\theta(x)=1$ for $x \geq 0$ or 0 otherwise. However, if one chooses to use Eq. (122) and divides the relation in Eq. (122) by $-q^{2}$ before applying the Borel transform, then the Gegenbauer moment sum rules are

$$
\begin{aligned}
& {\left[a_{l}^{\|,{ }^{3} P_{1}} f_{3}^{2} P_{1}\left(e^{-m_{3}^{2} P_{1} / M^{2}}-1\right)+a_{l}^{\|,{ }^{1} P_{1}} a_{0}^{\|,{ }^{1} P_{1}} f_{{ }^{2} P_{1}}^{2}\left(e^{-m_{1_{P_{1}}}^{2} / M^{2}}-1\right)\right] \frac{3(l+1)(l+2)}{2(2 l+3)} L^{-\gamma_{(l)} / \|}} \\
& =\frac{\alpha_{s}}{2 \pi^{3}} M^{2}\left[1-e^{-s_{0}^{\|} / M^{2}}-\frac{s_{0}}{M^{2}}\right] \int_{0}^{1} d u u \bar{u} C_{l}^{3 / 2}(2 u-1) \ln ^{2} \frac{u}{\bar{u}} \\
& -\frac{C_{l}^{3 / 2}(1)}{M^{2}}\left(m_{q_{1}}\left\langle\bar{q}_{2} q_{2}\right\rangle+m_{q_{2}}\left\langle\bar{q}_{1} q_{1}\right\rangle\right)+\frac{C_{l}^{3 / 2}(1)}{12 M^{2}}\left\langle\frac{\alpha_{s}}{\pi} G^{2}\right\rangle \\
& -\frac{1}{M^{4}} C_{l-1}^{5 / 2}(1) \theta(l-1)\left(m_{q_{1}}\left\langle\bar{q}_{2} g_{s} \sigma G q_{2}\right\rangle+m_{q_{2}}\left\langle\bar{q}_{1} g_{s} \sigma G q_{1}\right\rangle\right) L^{-14 /(3 b)} \\
& +\frac{16 \pi \alpha_{s}}{9 M^{4}} C_{l}^{3 / 2}(1)\left\langle\bar{q}_{1} q_{1}\right\rangle\left\langle\bar{q}_{2} q_{2}\right\rangle+\frac{16 \pi \alpha_{s}}{81 M^{4}}\left[C_{l}^{3 / 2}(1)\right]^{2}\left(\left\langle\bar{q}_{1} q_{1}\right\rangle^{2}+\left\langle\bar{q}_{2} q_{2}\right\rangle^{2}\right)
\end{aligned}
$$

for even $l \geq 2$ and

$$
\begin{aligned}
& {\left[a_{l}^{\|,{ }^{3} P_{1}} f_{{ }^{2} P_{1}}^{2}\left(e^{-m_{3_{P_{1}}}^{2} / M^{2}}-1\right)+a_{l}^{\|,{ }^{1} P_{1}} a_{0}^{\|,{ }^{1} P_{1}} f_{{ }^{2} P_{1}}^{2}\left(e^{-m_{1_{P_{1}}}^{2} / M^{2}}-1\right)\right] \frac{3(l+1)(l+2)}{2(2 l+3)} L^{-\gamma_{(l)}^{\|} / b}} \\
& =-\frac{C_{l}^{3 / 2}(1)}{M^{2}}\left(m_{q_{1}}\left\langle\bar{q}_{2} q_{2}\right\rangle-m_{q_{2}}\left\langle\bar{q}_{1} q_{1}\right\rangle\right) \\
& \quad-\frac{1}{M^{4}} C_{l-1}^{5 / 2}(1) \theta(l-1)\left(m_{q_{1}}\left\langle\bar{q}_{2} g_{s} \sigma G q_{2}\right\rangle-m_{q_{2}}\left\langle\bar{q}_{1} g_{s} \sigma G q_{1}\right\rangle\right) L^{-14 /(3 b)} \\
& \quad-\frac{16 \pi \alpha_{s}}{81 M^{4}}\left[C_{l}^{3 / 2}(1)\right]^{2}\left(\left\langle\bar{q}_{1} q_{1}\right\rangle^{2}-\left\langle\bar{q}_{2} q_{2}\right\rangle^{2}\right),
\end{aligned}
$$

for odd $l$ (of the $K_{1 A}$ meson), where we have substituted

$$
\tilde{I}_{1}^{(l)}(0)=\left[a_{l}^{\|,{ }^{3} P_{1}} f_{3^{2} P_{1}}^{2}+a_{l}^{\|,{ }^{1} P_{1}} a_{0}^{\|,{ }^{1} P_{1}}\right] \frac{3(l+1)(l+2)}{2(2 l+3)}-\frac{\alpha_{s}}{2 \pi^{3}} s_{0}^{\|} \int_{0}^{1} d u u \bar{u} C_{l}^{3 / 2}(2 u-1) \ln ^{2} \frac{u}{\bar{u}},
$$

which can be determined in the limit $M^{2} \rightarrow \infty$, into the above two equations.

Before we discuss the reliability of the sum rules about Eqs. (125), (126) and (127), (128), one should note that, for even $l$, the contributions due to ${ }^{1} P_{1}$ states are $\sim \mathcal{O}\left(m_{q}^{2}\right)$ and thus negligible. Nevertheless, for odd $l$, the contributions for $1^{3} P_{1}$ and $1^{1} P_{1}$ states are of the same order of magnitude. The quality of the sum rules in Eqs. (125) and (126) is not good due to the following two reasons. (i) The OPE series converges very slowly. For instance, if taking a close look at Eq. (125) with $l=2$, the terms of dimension-4 and -6 in the OPE series are still comparable to the perturbative contribution even for choosing a quite large Borel mass $M^{2} \sim 2 \mathrm{GeV}^{2}$. (ii) The terms of dimension-4 and -6 have the opposite sign compared with the perturbative contribution, such that the contributions of higher resonance states that we modeled have also the opposite sign compared with the lowest-lying state. As a result, it is difficult to choose a reliable windows for sum rules given in Eqs. (125) and (126). 
Nevertheless, the sum rules, given Eqs. (127) and (128), converge much more quickly. Meanwhile, in the OPE series in Eq. (127) the contribution of the perturbative term have the same sign as the the terms of dimension- 4 and -6 . Consequently, we can find a suitable Borel window, where the contributions originating from higher resonances and the highest OPE terms are well under control. For the time being, we will hence focus on Eqs. (127) and (128). The numerical results are given in Sec. 4.4.3. Considering Eq. (128) with $l=1$ for the $K_{1 A}$ (and $K_{1 B}$ ) mesons, the result approximately reads

$$
\begin{gathered}
a_{1}^{\|, K_{1 A}}+a_{1}^{\|, K_{1 B}} a_{0}^{\|, K_{1 B}} \frac{f_{K_{1 B}}^{2}}{f_{K_{1 A}}^{2}} \simeq \frac{5}{9} \frac{1}{f_{K_{1 A}}^{2}\left(e^{-\bar{m}^{2} / M^{2}}-1\right)} L^{\gamma_{(1)}^{\|} / b}\left\{-\frac{3}{M^{2}}\left(m_{s}\left\langle\bar{q}_{2} q_{2}\right\rangle-m_{q_{2}}\langle\bar{s} s\rangle\right)\right. \\
\left.-\frac{1}{M^{4}}\left(m_{s}\left\langle\bar{q}_{2} g_{s} \sigma G q_{2}\right\rangle-m_{q_{2}}\left\langle\bar{s} g_{s} \sigma G s\right\rangle\right) L^{-14 /(3 b)}-\frac{16 \pi \alpha_{s}}{9 M^{4}}\left(\langle\bar{s} s\rangle^{2}-\left\langle\bar{q}_{2} q_{2}\right\rangle^{2}\right)\right\},
\end{gathered}
$$

with $\bar{m}=\left(m_{K_{1 A}}+m_{K_{1 B}}\right) / 2$.

\subsubsection{Gegenbauer moments of $\Phi_{\perp}^{3} P_{1}$ for $1^{3} P_{1}$ mesons}

To calculate the Gegenbauer moments of $\Phi_{\perp}^{3} P_{1}$ for $1^{3} P_{1}$ states, we consider the following correlation function

$$
\int d^{4} x e^{i q x}\langle 0| \mathrm{T}\left(\Omega_{\mu}^{\perp(l)}(x) O_{\nu}^{\dagger}(0)|0\rangle=\left[g_{\mu \nu}-\frac{1}{q \bar{z}}\left(q_{\mu} \bar{z}_{\nu}+q_{\nu} \bar{z}_{\mu}\right)\right](q \bar{z})^{l+1} T_{1}\left(q^{2}\right)+\cdots,\right.
$$

where

$$
O_{\nu}(0)=\overline{q_{1}}(0) \gamma_{\mu} \gamma_{5} q_{2}(0)
$$

and, to leading logarithmic accuracy, the relevant multiplicatively renormalizable operator is

$$
\Omega_{\mu}^{\perp(l)}(x)=\sum_{j=0}^{l} c_{n, j}(i \bar{z} \partial)^{l-j} \bar{q}_{2}(x) \sigma_{\mu \alpha} \gamma_{5} \bar{z}^{\alpha}(i \bar{z} \stackrel{\leftrightarrow}{D})^{j} q_{1}(x)
$$

with $\bar{z}$ being the light-like vector as defined previously. $\Omega_{\mu}^{\perp(l)}$ and $O_{\nu}$ satisfy the relation:

$$
\begin{aligned}
& \sum_{\lambda}\left\langle 0\left|\Omega_{\mu}^{\perp(l)}(0)\right| 1^{3} P_{1}(P, \lambda)\right\rangle\left\langle 1^{3} P_{1}(P, \lambda)\left|O_{\nu}(0)\right| 0\right\rangle \\
& =i f_{{ }^{3} P_{1}}^{\perp} f_{3 P_{1}} m_{3} P_{1} \frac{3(l+1)(l+2)}{2(2 l+3)}\left[g_{\mu \nu}-\frac{1}{P \bar{z}}\left(P_{\mu} \bar{z}_{\nu}+P_{\nu} \bar{z}_{\mu}\right)\right](P \bar{z})^{l+1} a_{l}^{\perp,{ }^{3} P_{1}}+\cdots .
\end{aligned}
$$

Using the dispersion relation for $T_{1}, a_{l}^{\perp,}{ }^{3} P_{1}$ can be represented in the form

$$
\frac{3(l+1)(l+2)}{2(2 l+3)} \frac{f_{{ }_{3} P_{1}}^{\perp} f_{3} P_{1} m_{3} P_{1} a_{l}^{\perp,},{ }^{3} P_{1}}{m^{2}{ }^{2}-q_{1}}=\frac{1}{\pi} \int_{0}^{s_{0}^{\perp,{ }^{3} P_{1}}} d s \frac{\operatorname{Im} T_{1}^{\mathrm{OPE}}(s)}{s-q^{2}}
$$


where

$$
\begin{aligned}
T_{1}^{\mathrm{OPE}}(0)= & \frac{3}{4 \pi^{2}} \ln \frac{-q^{2}}{\mu^{2}}\left(\int_{0}^{1} d \alpha\left[m_{q_{2}} \alpha+m_{q_{1}}(\alpha-1)\right] C_{l}^{3 / 2}(2 \alpha-1)\right) \\
& -\frac{1}{q^{2}} C_{l}^{3 / 2}(1)\left(\left\langle\bar{q}_{2} q_{2}\right\rangle+\left\langle\bar{q}_{1} q_{1}\right\rangle(-1)^{l+1}\right) \\
& +\left[\frac{1}{3} C_{l}^{3 / 2}(1)+2 C_{l-1}^{5 / 2}(1) \theta(l-1)\right] \frac{\left\langle\bar{q}_{2} g_{s} \sigma \cdot G q_{2}\right\rangle+\left\langle\bar{q}_{1} g_{s} \sigma \cdot G q_{1}\right\rangle(-1)^{l+1}}{q^{4}} \\
& \left.-\frac{2 \pi^{2}}{3 q^{6}}\left[20 C_{l-2}^{7 / 2}(1) \theta(l-2)+C_{l-1}^{5 / 2}(1) \theta(l-1)\right)\right]\left\langle\frac{\alpha_{s}}{\pi} G^{2}\right\rangle\left[\left\langle\bar{q}_{2} q_{2}\right\rangle+\left\langle\bar{q}_{1} q_{1}\right\rangle(-1)^{l+1}\right] .
\end{aligned}
$$

Since ${ }^{1} P_{1}$ states can have small axial-vector coupling constants due the G-parity violating (SU(3)-breaking) effect, the RG-improved sum rules for Gegenbauer moments $a_{l}^{\perp,{ }^{3} P_{1}}$ thus read

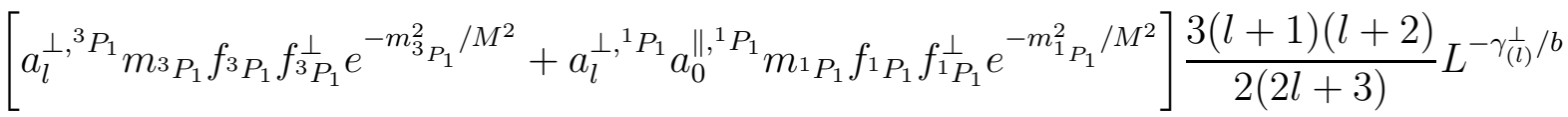

$$
\begin{aligned}
& =-\left\{\frac{3}{4 \pi^{2}} M^{2}\left(1-e^{-s_{0}^{\perp,},{ }^{3} P_{1}} / M^{2}\right)\left(\int_{0}^{1} d \alpha C_{l}^{3 / 2}(2 \alpha-1)\left[m_{q_{2}} \alpha+m_{q_{1}}(\alpha-1)\right]\right) L^{-4 / b}\right. \\
& -C_{l}^{3 / 2}(1)\left(\left\langle\bar{q}_{2} q_{2}\right\rangle+\left\langle\bar{q}_{1} q_{1}\right\rangle(-1)^{l+1}\right) L^{4 / b} \\
& -\left[\frac{1}{3} C_{l}^{3 / 2}(1)+2 C_{l-1}^{5 / 2}(1) \theta(l-1)\right] \frac{\left\langle\bar{q}_{2} g_{s} \sigma \cdot G q_{2}\right\rangle+\left\langle\bar{q}_{1} g_{s} \sigma \cdot G q_{1}\right\rangle(-1)^{l+1}}{M^{2}} L^{-2 /(3 b)} \\
& \left.-\frac{\pi^{2}}{M^{4}}\left[\frac{20}{3} C_{l-2}^{7 / 2}(1) \theta(l-2)+\frac{1}{3} C_{l-1}^{5 / 2}(1) \theta(l-1)\right)\right] \\
& \left.\times\left\langle\frac{\alpha_{s}}{\pi} G^{2}\right\rangle\left(\left\langle\bar{q}_{2} q_{2}\right\rangle+\left\langle\bar{q}_{1} q_{1}\right\rangle(-1)^{l+1}\right) L^{4 / b}\right\},
\end{aligned}
$$

where for odd $l$ the corrections coming from the ${ }^{1} P_{1}$ states are of order $m_{q}^{2}$ and can be neglected, whereas for even $l$ the $1^{3} P_{1}$ and $1^{1} P_{1}$ states give contributions of the same order. With $l=0,2$, we can therefore obtain the following approximation for the $K_{1 A}$ and $K_{1 B}$ mesons:

$$
\begin{aligned}
a_{0}^{\perp, K_{1 A}}+ & a_{0}^{\|, K_{1 B}} \frac{m_{K_{1 B}} f_{K_{1 B}}^{\perp} f_{K_{1 B}}}{m_{K_{1 A}} f_{K_{1 A}}^{\perp} f_{K_{1 A}}} \simeq-\frac{1}{m_{K_{1 A}} f_{K_{1 A}}^{\perp} f_{K_{1 A}}} e^{\bar{m}^{2} / M^{2}} L^{\gamma_{(0)}^{\perp} / b} \\
& \times\left\{\frac{3}{8 \pi^{2}} M^{2}\left(1-e^{-s_{0} / M^{2}}\right)\left(m_{q_{2}}-m_{s}\right) L^{-4 / b}-\left(\left\langle\bar{q}_{2} q_{2}\right\rangle-\langle\bar{s} s\rangle\right) L^{4 / b}\right. \\
& \left.-\frac{\left\langle\bar{q}_{2} g_{s} \sigma \cdot G q_{2}\right\rangle-\left\langle\bar{s} g_{s} \sigma \cdot G s\right\rangle}{3 M^{2}} L^{-2 /(3 b)}+\frac{0}{M^{4}}\left\langle\frac{\alpha_{s}}{\pi} G^{2}\right\rangle\left(\left\langle\bar{q}_{2} q_{2}\right\rangle-\langle\bar{s} s\rangle\right) L^{4 / b}\right\},
\end{aligned}
$$




$$
\begin{aligned}
a_{2}^{\perp, K_{1 A}} & +a_{2}^{\perp, K_{1 B}} a_{0}^{\|, K_{1 B}} \frac{m_{K_{1 B}} f_{K_{1 B}}^{\perp} f_{K_{1 B}}}{m_{K_{1 A}} f_{K_{1 A}}^{\perp} f_{K_{1 A}}} \simeq-\frac{7}{18} \frac{1}{m_{K_{1 A}} f_{K_{1 A}}^{\perp} f_{K_{1 A}}} e^{\bar{m}^{2} / M^{2}} L^{\gamma_{(2)}^{\perp} / b} \\
\times & \left\{\frac{3}{8 \pi^{2}} M^{2}\left(1-e^{-s_{0} / M^{2}}\right)\left(m_{q_{2}}-m_{s}\right) L^{-4 / b}-6\left(\left\langle\bar{q}_{2} q_{2}\right\rangle-\langle\bar{s} s\rangle\right) L^{4 / b}\right. \\
& \left.-12 \frac{\left\langle\bar{q}_{2} g_{s} \sigma \cdot G q_{2}\right\rangle-\left\langle\bar{s} g_{s} \sigma \cdot G s\right\rangle}{M^{2}} L^{-2 /(3 b)}-\frac{25}{3} \frac{\pi^{2}}{M^{4}}\left\langle\frac{\alpha_{s}}{\pi} G^{2}\right\rangle\left(\left\langle\bar{q}_{2} q_{2}\right\rangle-\langle\bar{s} s\rangle\right) L^{4 / b}\right\}
\end{aligned}
$$

with $s_{0} \approx s_{0}^{\perp, K_{1 A}}$.

\subsubsection{Results}

In the numerical analysis, we shall adopt parameters which are collected in Appendix $\mathrm{C}$ and Tables 2 and 3. It should be noted that for the moment sum rules the actual expansion parameter is $M^{2} / l$ in the large $l$ limit [7]. As a result, for $a_{l}^{\|}$and $a_{l}^{\perp}$ with a larger $l$ and fixed $M^{2}$, the OPE series are convergent more slowly or even divergent as compared with the sum rules for the masses or decay constants.

Consider $a_{l}^{\|,{ }^{3} P_{1}}$ first. We adopt the sum rules given in Eqs. (127) and (128). As discussed after Eq. (128), these two sum rules can give much more reliable results than those in Eqs. (125) and (126). For $a_{2}^{\|,{ }^{3} P_{1}}$, as expected, we find the higher Borel window to be $2.0 \mathrm{GeV}^{2}<M^{2}<3.0 \mathrm{GeV}^{2}$, where the contributions originating from higher resonance states lie between $15 \%$ and $32 \%$, and moreover the correction arising from the highest dimension term in OPE series is between $17 \%$ and $6 \%$. For $a_{1}^{\|, K_{1 A}}+a_{1}^{\|, K_{1 B}} a_{0}^{\|, K_{1 B}} f_{K_{1 B}}^{2} / f_{K_{1 A}}^{2}$, the correction from highest dimension is quite small, but it is hard to estimate the contributions from higher resonances. Fortunately, the result of this sum rule is quite stable and we will choose to use the Borel window $1.5 \mathrm{GeV}^{2}<M^{2}<2.5 \mathrm{GeV}^{2}$, which is in between the cases of the sum rules for decay constants and $a_{2}^{\|,{ }^{3} P_{1}}$.

The sum rules for $a_{1}^{\perp,{ }^{3} P_{1}}$ are the typical cases about the non-diagonal sum rules, for which the main contributions in OPE may come from the quark and quark-gluon condensates. It has been argued in Refs. [36, 37, 38, that these sum rules may suffer from contributions of higher resonances, so that the value about the lowest-lying meson may be overestimate. On the other hand, note that usually the radiative corrections are constructive at the $10 \%$ level for each OPE term. Since we have neglected the radiative corrections, it means that the real value for the lowest-lying meson may be underestimate. The above two corrections may partially cancel each other. Equivalently, these two corrections can be lumped into the uncertainties of the condensates and quark masses. In summary, the non-diagonal sum rules may suffer from above two corrections and the net effects on results may be less than $10 \%$. Essentially, the above estimate is suitable for all twist-3 and twist-3 parameters studied in the present paper. However, since we do not do the qualitative estimates about the parameters, we thus do not include these possible errors in Tables 4, 5, and 6.

The best stability of the $a_{1}^{\perp,{ }^{3} P_{1}}$ sum rules is reached within the Borel window $1.5 \mathrm{GeV}^{2}<$ $M^{2}<2.5 \mathrm{GeV}^{2}$, the same as the case of $a_{1}^{\|, K_{1 A}}$, where the correction from the highest 
dimension term at the quark-gluon level is quite small. For G-parity violating Gegenbauer moments, $a_{0}^{\perp, K_{1 A}}+c_{2} \cdot a_{0}^{\|, K_{1 B}}$ and $a_{2}^{\perp, K_{1 A}}+c_{2} \cdot a_{2}^{\perp, K_{1 B}} a_{0}^{\|, K_{1 B}}$ with

$$
c_{2}=\left(m_{K_{1 B}} / m_{K_{1 A}}\right)\left(f_{K_{1 B}}^{\perp} f_{K_{1 B}} / f_{K_{1 A}}^{\perp} f_{K_{1 A}}\right),
$$

we find the suitable Borel windows to be $1.3 \mathrm{GeV}^{2}<M^{2}<2.0 \mathrm{GeV}^{2}$ and $2.0 \mathrm{GeV}^{2}<M^{2}<$ $3.0 \mathrm{GeV}^{2}$, respectively. Besides, the contributions originating from higher resonances (about $15 \% \sim 32 \%$ and $15 \% \sim 32 \%$ for the former and latter ones, respectively) and the highest OPE terms (zero and $5 \% \sim 2 \%$ for the former and latter ones, respectively) are well under control.

For G-parity invariant parameters involving quark mass corrections, because the results may be sensitive to the nonperturbative parameters for which we have considered larger uncertainties of the parameters here (see Appendix (C)), we therefore re-examine the errors of $1^{1} P_{1}$ states as well. The formulas for $1^{1} P_{1}$ states were given in Ref. [18]. The results for G-parity invariant Gegenbauer moments for $1^{1} P_{1}$ and $1^{3} P_{1}$ states are summarized in Table 4. Note that the results for $h_{1}$ and $h_{8}$ (the $1^{1} P_{1}$ states) are new, where use of $a_{2}^{\perp, \omega_{1}} \approx a_{2}^{\perp, \omega_{8}} \approx a_{2}^{\perp, \phi}$ has been made in the numerical analysis and $a_{2}^{\perp, \phi}=0.0 \pm 0.1$ [39]. To exhibit the quality of the sum rules for the $1^{3} P_{1}$ results, the Gegenbauer moments versus the Borel mass squared are plotted in Figs. 2 and 3 ,

For G-parity violating parameters, the Gegenbauer moments for $K_{1 A}$ and $K_{1 B}$ mix as given in Eqs. (129), (137) and (138). On the other hand, the G-parity violating quantities were not considered correctly in Ref. [18] owing to the mentioned mixtures and should read

$$
\begin{gathered}
a_{1}^{\perp, K_{1 B}}+a_{1}^{\perp, K_{1 A}} a_{0}^{\perp, K_{1 A}} \frac{\left(f_{K_{1 A}}^{\perp}\right)^{2}}{\left(f_{K_{1 B}}^{\perp}\right)^{2}} \simeq \frac{5}{9} \frac{1}{\left(f_{K_{1 A}}^{\perp}\right)^{2}} e^{\bar{m}^{2} / M^{2}} L^{\gamma_{(1)}^{\|} / b}\left\{-\frac{3}{M^{2}}\left(m_{s}\left\langle\bar{q}_{2} q_{2}\right\rangle-m_{q_{2}}\langle\bar{s} s\rangle\right)\right. \\
\left.-\frac{2}{M^{4}}\left(m_{s}\left\langle\bar{q}_{2} g_{s} \sigma G q_{2}\right\rangle-m_{q_{2}}\left\langle\bar{s} g_{s} \sigma G s\right\rangle\right) L^{-14 /(3 b)}-\frac{9}{5}\left(f_{K^{*}}^{\perp}\right)^{2} a_{1}^{\perp, K^{*}} e^{-m_{K^{*}}^{2} / M^{2}}\right\},
\end{gathered}
$$

and

$$
\begin{aligned}
a_{2}^{\|, K_{1 B}} & +a_{2}^{\|, K_{1 A}} a_{0}^{\perp, K_{1 A}} \frac{m_{K_{1 A}} f_{K_{1 A}}^{\perp} f_{K_{1 A}}}{m_{K_{1 B}} f_{K_{1 B}}^{\perp} f_{K_{1 B}}} \simeq-\frac{7}{18} \frac{1}{m_{K_{1 B}} f_{K_{1 B}}^{\perp} f_{K_{1 B}}} e^{\bar{m}^{2} / M^{2}} L^{\gamma_{(2)}^{\perp} / b} \\
\times & \left\{\frac{3}{8 \pi^{2}} M^{2}\left(1-e^{-s_{0} / M^{2}}\right)\left(m_{q_{2}}-m_{s}\right) L^{-4 / b}-6\left(\left\langle\bar{q}_{2} q_{2}\right\rangle-\langle\bar{s} s\rangle\right) L^{4 / b}\right. \\
& \left.-12 \frac{\left\langle\bar{q}_{2} g_{s} \sigma \cdot G q_{2}\right\rangle-\left\langle\bar{s} g_{s} \sigma \cdot G s\right\rangle}{M^{2}} L^{-2 /(3 b)}-\frac{25}{3} \frac{\pi^{2}}{M^{4}}\left\langle\frac{\alpha_{s}}{\pi} G^{2}\right\rangle\left(\left\langle\bar{q}_{2} q_{2}\right\rangle-\langle\bar{s} s\rangle\right) L^{4 / b}\right\} .
\end{aligned}
$$

We use the updated values $a_{1}^{\perp, K^{*}}(1 \mathrm{GeV})=0.04 \pm 0.03$ [40] and $f_{K^{*}}^{\perp}=(0.185 \pm 0.010) \mathrm{GeV}$ [40] in the numerical analysis. Eqs. (129), (137), (138), (139), and (140) do not offer sufficient relations to obtain explicit solutions for G-parity violating parameters but can give the following relations (at the scale $\mu=1 \mathrm{GeV}$ ):

$$
a_{1}^{\|, K_{1 A}}-(1.10 \pm 0.30) a_{0}^{\|, K_{1 B}}=-0.15 \pm 0.06
$$




$$
\begin{aligned}
& a_{0}^{\perp, K_{1 A}}+(0.59 \pm 0.15) a_{0}^{\|, K_{1 B}}=0.17 \pm 0.11, \\
& a_{1}^{\perp, K_{1 B}}-(1.87 \pm 0.56) a_{0}^{\perp, K_{1 A}}=0.02 \pm 0.08, \\
& a_{2}^{\perp, K_{1 A}}-(0.01 \pm 0.16) a_{0}^{\|, K_{1 B}}=0.02 \pm 0.18 \\
& a_{2}^{\|, K_{1 B}}-(0.08 \pm 0.07) a_{0}^{\perp, K_{1 A}}=0.01 \pm 0.09,
\end{aligned}
$$

where we have substituted the numerical values of mesons' masses and decay constants given in Tables 2 and 3. If the above G-parity violating parameters are expected to be small as the results for $K$ and $K^{*}$ (for instance, see results in Ref. [40]), it is preferred that $a_{0}^{\perp, K_{1 A}}$ and $a_{0}^{\|, K_{1 B}}$ have the same positive sign. On the other hand, comparing Eq. (136) in this paper and Eq. (3.23) in Ref. [18, we obtain the good approximation between G-parity invarint Gegenbauer moments (with odd $l$ ) of $1^{3} P_{1}$ and $1^{1} P_{1}$ states:

$$
a_{l}^{\perp,{ }^{3} P_{1}} \simeq a_{l}^{\|,{ }^{1} P_{1}} \frac{m_{1 P_{1}} f^{1} P_{1} f_{{ }^{1} P_{1}}^{\perp}}{m_{3 P_{1}} f^{3} P_{1} f^{\perp} P_{1}},
$$

which can be reconfirmed from the results in Table 4. Therefore, we further assume that the G-parity violating Gegenbauer moments satisfy a similar relation with an enlarged uncertainty range:

$$
\frac{a_{0}^{\perp, K_{1 A}}}{a_{0}^{\|, K_{1 B}} \frac{m_{K_{1 B}} f_{K_{1 B}} f_{K_{1 B}}^{\perp}}{m_{K_{1 A}} f_{K_{1 A}} f_{K_{1 A}}^{\perp}}}=1.0 \pm 0.3 .
$$

From the above estimate, it follows

$$
\begin{array}{ll}
a_{0}^{\perp, K_{1 A}}=0.08 \pm 0.09, & a_{0}^{\|, K_{1 B}}=0.14 \pm 0.15 \\
a_{1}^{\|, K_{1 A}}=0.00 \pm 0.26, & a_{1}^{\perp, K_{1 B}}=0.17 \pm 0.22 \\
a_{2}^{\perp, K_{1 A}}=0.02 \pm 0.20, & a_{2}^{\|, K_{1 B}}=0.02 \pm 0.10
\end{array}
$$

which are collected in Table 4,

Finally, four remarks are in order. First, we will simply take $f_{3}^{\perp} P_{1}=f_{3} P_{1}$, which is independent of the scale, in the study since only the products of $f_{3 P_{1}}^{\perp} a_{l}^{\perp,}{ }^{3} P_{1}$ are relevant. Second, the sum rules obtained from the nondiagonal correlation functions in Eq. (130) can also determine the sign of $f_{3}^{\perp} P_{1} a_{1}^{\perp,}{ }^{3} P_{1}$ relative to $f_{3} P_{1}$. Third, for the present case, the RG effects are relatively small compared with the uncertainties of input parameters. Fourth, neglecting the small isospin violation but considering the SU(3)-breaking correction, $a_{1}^{\|}, a_{3}^{\|}$ $a_{0}^{\perp}$ and $a_{2}^{\perp}$ are nonzero only for $K_{1 A}$. Note that here we adopt the convention that $q_{1} \equiv s$ for $K_{1 A}$ and $K_{1 B}$. For $K_{1 A}\left(K_{1 B}\right)$ containing an $\bar{s}$ quark, we have the following replacements $a_{1}^{\|}, a_{0,2}^{\perp} \rightarrow-a_{1}^{\|},-a_{0,2}^{\perp}\left(a_{0,2}^{\|}, a_{1}^{\perp} \rightarrow-a_{0,2}^{\|},-a_{1}^{\perp}\right)$ for G-parity violating parameters. 
Table 4: Gegenbauer moments of $\Phi_{\perp}$ and $\Phi_{\|}$for $1^{3} P_{1}$ and $1^{1} P_{1}$ mesons, where uses of $f_{3 P_{1}}^{\perp}=f_{3} P_{1}$ and $f_{{ }^{1} P_{1}}=f_{{ }^{1} P_{1}}^{\perp}(1 \mathrm{GeV})$ have been made. For $1^{1} P_{1}$ states, the results for $h_{1}$ and $h_{8}$ are new, for $a_{0,2}^{\|, K_{1 B}}$ and $a_{1}^{\perp, K_{1 B}}$ are corrected, and for the rest are updated.

\begin{tabular}{|c|c|c|c|c|c|c|}
\hline$\mu$ & $a_{2}^{\|, a_{1}(1260)}$ & $a_{2}^{\|, f_{1}{ }^{3} P_{1}}$ & $a_{2}^{\|, f_{8}{ }^{3} P_{1}}$ & $a_{2}^{\|, K_{1 A}}$ & \multicolumn{2}{|c|}{$a_{1}^{\|, K_{1 A}}$} \\
\hline $1 \mathrm{GeV}$ & $-0.02 \pm 0.02$ & $-0.04 \pm 0.03$ & $-0.07 \pm 0.04$ & $-0.05 \pm 0.03$ & \multicolumn{2}{|c|}{$0.00 \pm 0.26$} \\
$2.2 \mathrm{GeV}$ & $-0.01 \pm 0.01$ & $-0.03 \pm 0.02$ & $-0.05 \pm 0.03$ & $-0.04 \pm 0.02$ & \multicolumn{2}{|c|}{$0.00 \pm 0.22$} \\
\hline \hline$\mu$ & $a_{1}^{\perp, a_{1}(1260)}$ & $a_{1}^{\perp, f_{1}{ }^{P_{1}}}$ & $a_{1}^{\perp, f_{8}{ }^{P_{1}}}$ & $a_{1}^{\perp, K_{1 A}}$ & $a_{0}^{\perp, K_{1 A}}$ & $a_{2}^{\perp, K_{1 A}}$ \\
\hline $1 \mathrm{GeV}$ & $-1.04 \pm 0.34$ & $-1.06 \pm 0.36$ & $-1.11 \pm 0.31$ & $-1.08 \pm 0.48$ & $0.08 \pm 0.09$ & $0.02 \pm 0.20$ \\
$2.2 \mathrm{GeV}$ & $-0.85 \pm 0.28$ & $-0.86 \pm 0.29$ & $-0.90 \pm 0.25$ & $-0.88 \pm 0.39$ & $0.07 \pm 0.08$ & $0.01 \pm 0.15$ \\
\hline \hline$\mu$ & $a_{1}^{\|, b_{1}(1235)}$ & $a_{1}^{\|, h_{1}{ }^{P_{1}}}$ & $a_{1}^{\|, h_{8}{ }^{P_{1}}}$ & $a_{1}^{\|, K_{1 B}}$ & $a_{0}^{\|, K_{1 B}}$ & $a_{2}^{\|, K_{1 B}}$ \\
\hline $1 \mathrm{GeV}$ & $-1.95 \pm 0.35$ & $-2.00 \pm 0.35$ & $-1.95 \pm 0.35$ & $-1.95 \pm 0.45$ & $0.14 \pm 0.15$ & $0.02 \pm 0.10$ \\
$2.2 \mathrm{GeV}$ & $-1.61 \pm 0.29$ & $-1.65 \pm 0.29$ & $-1.61 \pm 0.29$ & $-1.61 \pm 0.37$ & $0.14 \pm 0.15$ & $0.01 \pm 0.07$ \\
\hline \hline$\mu$ & $a_{2}^{\perp, b_{1}(1235)}$ & $a_{2}^{\perp, h_{1}{ }^{P_{1}}}$ & $a_{2}^{\perp, h_{8}{ }^{P_{1}}}$ & $a_{2}^{\perp, K_{1 B}}$ & $a_{1}^{\perp, K_{1 B}}$ \\
\hline $1 \mathrm{GeV}$ & $0.03 \pm 0.19$ & $0.18 \pm 0.22$ & $0.14 \pm 0.22$ & $-0.02 \pm 0.22$ & $0.17 \pm 0.22$ \\
$2.2 \mathrm{GeV}$ & $0.02 \pm 0.15$ & $0.14 \pm 0.17$ & $0.11 \pm 0.17$ & $-0.02 \pm 0.17$ & $0.14 \pm 0.18$ \\
\hline
\end{tabular}

\section{Determinations of three-parton LCDAs of twist-three}

In this section, using the QCD sum rule approach, we estimate the relevant parameters involving SU(3)-breaking effects in determinations of the three-parton LCDAs of twist-three. The quark masses (SU(3)-breaking effects), can give contributions not only to G-parity invariant conformal moments but also G-parity violating ones of LCDAs. In the calculation, the OPE series in the QCD sum rules are evaluated up to dimension-seven, but up to dimension-four for terms proportional to the quark masses.

The parameters can be calculated from the standard QCD sum rule approach by adopting a relevant two-point correlation function as the starting point. The choices of suitable interpolating currents may affect the qualities of the final estimates. Note again that in $\mathrm{SU}(3)$ limit the decay constant for a $1^{3} P_{1}$ state transiting to the vacuum via the local (pseudo-)tensor current vanishes due to the G-parity mismatch between the current and 

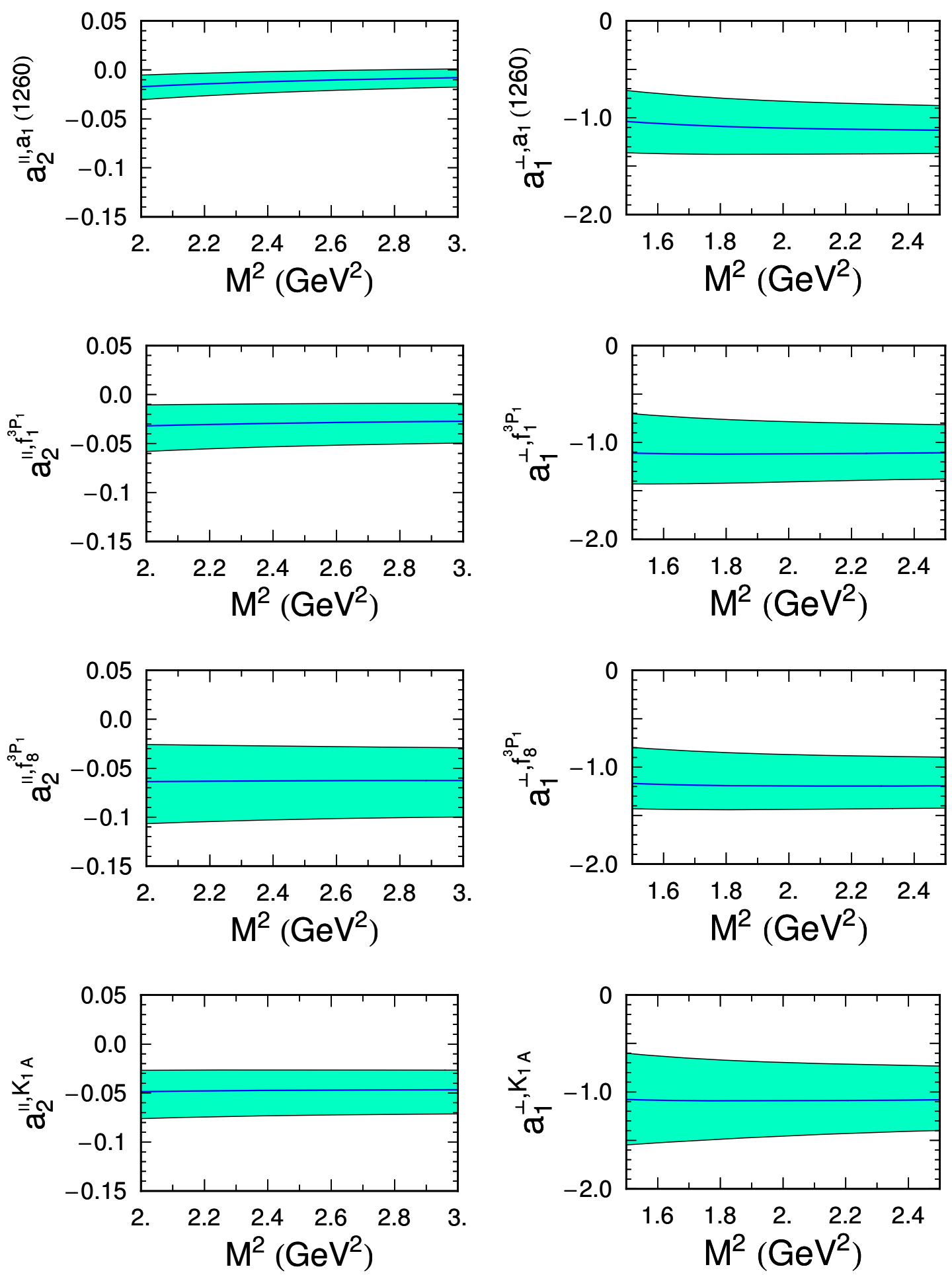

Figure 2: G-parity invariant Gegenbauer moments of leading twist LCDAs for $1^{3} P_{1}$ states, corresponding to the scale $\mu=1 \mathrm{GeV}$, as functions of the Borel mass squared. The solid curves and bands correspond to the central values and uncertainties of the input parameters, respectively. 

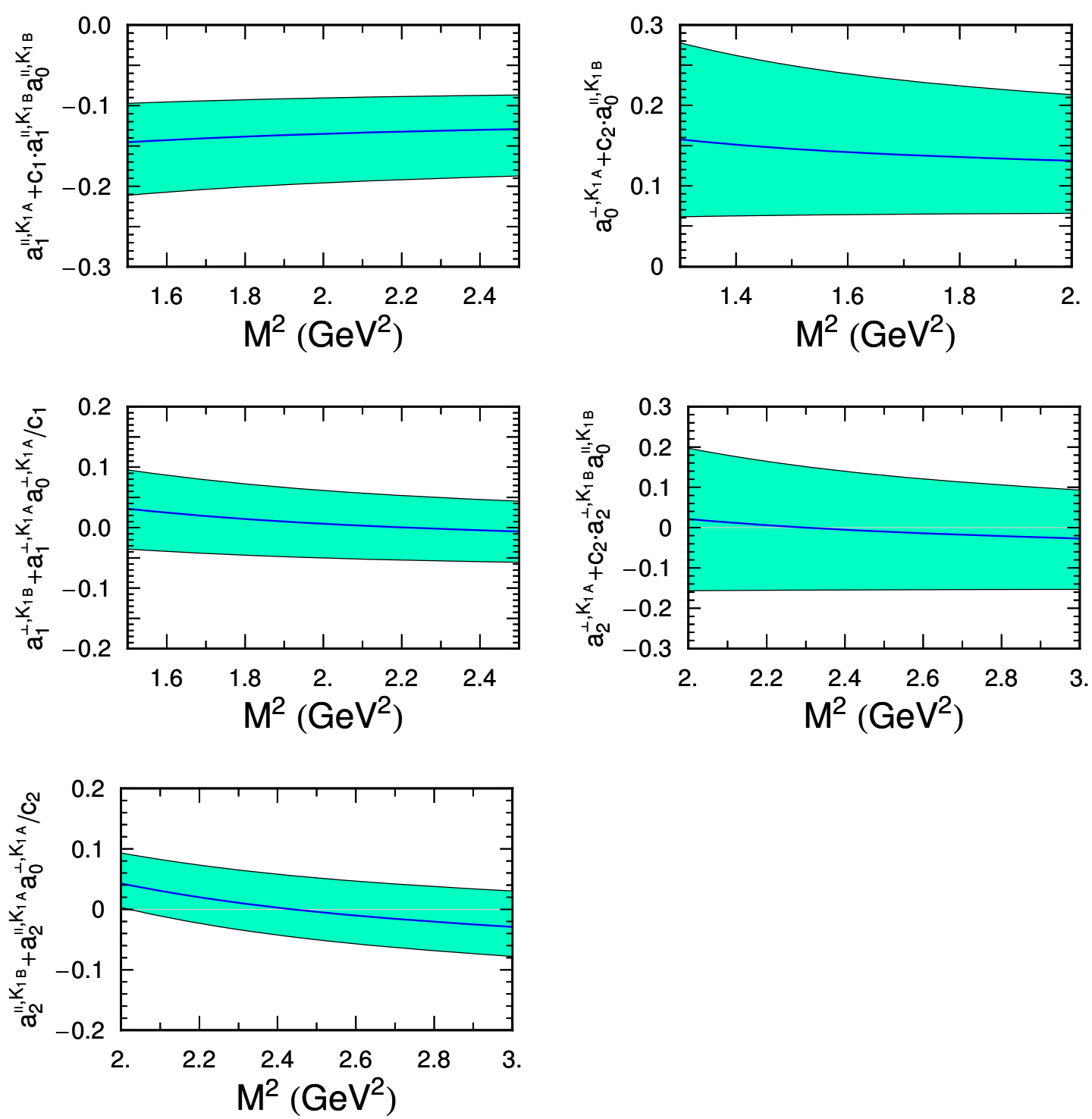

Figure 3: G-parity violating Gegenbauer moments of leading twist LCDAs, corresponding to the scale $\mu=1 \mathrm{GeV}$, as functions of the Borel mass squared, where $c_{1}=f_{K_{1 B}}^{2} / f_{K_{1 A}}^{2}$ and $c_{2}=\left(m_{K_{1 B}} / m_{K_{1 A}}\right)\left(f_{K_{1 B}}^{\perp} f_{K_{1 B}} / f_{K_{1 A}}^{\perp} f_{K_{1 A}}\right)$. The solid curves and bands correspond to the central values and uncertainties of the input parameters, respectively.

states, whereas the axial-vector decay constant vanishes for a $1^{1} P_{1}$ meson. Motivated from the above properties and to determine not only the magnitudes but also the relative signs for parameters relevant to three-parton LCDAs of twist-3, we thus choose one of the interpolating currents in the two-point correlation functions to be the local axial-vector (or pseudo-tensor) current to calculate the parameters for the $1^{3} P_{1}$ (or $1^{1} P_{1}$ ) state, i.e., we consider the nondiagonal correlation functions here. We did not consider the diagonal correlation functions 
in the beginning since it cannot determine the sign 9 of the parameters and cannot be used to evaluate the G-parity violating parameters due to the mixes of the $1^{3} P_{1}$ and $1^{1} P_{1}$ states.

Unlike results from diagonal correlation functions, where the perturbative contribution may dominate in the OPE series, our results show that the leading contributions are dominated by the term with the quark or gluon condensate in the most cases, and we may need to take into account a higher Borel window, so that the contribution originating from the highest dimension term in the OPE expansion can be well under control. However, a problem occurs in the $f_{3,{ }^{3} P_{1}}^{\perp}$ study for which the quark condensate contributions vanish in $\mathcal{O}\left(\alpha_{s}\right)$ after adding all the diagrams, and therefore the OPE result become highly unreliable (see the details in Sec. 5.1.3). We therefore resort to the diagonal sum rule for pursuing this parameter. However the lowest lying pseudoscalar meson contributes to the diagonal sum rule. Fortunately, although the pseudoscalar meson contribution is involved in the diagonal sum rule, such effects can be subtracted by using the non-diagonal sum rule for the pseudoscalar meson. These two sum rules have been studied in Ref. [20] for calculating the twist-3 parameter $f_{3 K}$ relevant to the twist-3 three-parton LCDA of the kaon. Instead, we use these two sum rules to extract the value of $f_{3,{ }^{3} P_{1}}^{\perp}$.

We study the parameters relevant to twist-3 three-parton LCDAs for $1^{3} P_{1}$ and $1^{1} P_{1}$ states in Secs. 5.1 and 5.2, respectively. One should note that, as calculating the sum rules for G-parity symmetric parameters of twist-3 three-parton LCDAs of $1^{3} P_{1}\left(1^{1} P_{1}\right)$ mesons, the G-parity violating parameters for $1^{1} P_{1}\left(1^{3} P_{1}\right)$ states, relatively suppressed by $\mathcal{O}\left(m_{q}^{2}\right)$, contribute to them but can be negligible. However, the sum rules for G-parity violating parameters for $1^{3} P_{1}\left(1^{1} P_{1}\right)$ mesons receive the contributions arising from G-parity symmetric parameters about $1^{1} P_{1}\left(1^{3} P_{1}\right)$ states, which are of the same order of magnitude because the axial-vector decay constants (pseudo-tensor decay constants) for $1^{1} P_{1}\left(1^{3} P_{1}\right)$ states do not vanish due to the quark mass corrections. The numerical results are given in Sec. 5.3, To LO approximation and including the quark mass corrections, the complete RG evolutions of the parameters are given in Appendix C.

\subsection{Axial-vector mesons with quantum number $1^{3} P_{1}$}

5.1.1 $f_{3,{ }^{3} P_{1}}^{V}, \omega_{3 P_{1}}^{V}$ and $\sigma_{3 P_{1}}^{V}$

The coupling constants $f_{3,{ }^{3} P_{1}}^{V}, \omega_{3 P_{1}}^{V}$ and $\sigma_{3 P_{1}}^{V}$ can be obtained by considering the correlation functions,

$$
i \int d^{4} x e^{i q x}\left\langle 0\left|T\left\{J_{1, \mu}^{3, V}(x), \bar{q}_{1}(0) \gamma_{\nu} \gamma_{5} q_{2}(0)\right\}\right| 0\right\rangle=-T_{3 P_{1}}^{V}\left(q^{2}\right)(q \bar{z})^{2} g_{\mu \nu}^{\perp}+\cdots
$$

\footnotetext{
${ }^{9}$ It is a little different for the definition of the diagonal correlation function here and in Ref. 20. In Ref. 20] and related studies, the authors choose the non-local light-ray operators in calculating the correlation functions. According to their definition, although some parameters' signs can be determined in the diagonal sum rules which are actually non-diagonal in our definition, the relative signs of $f_{3, A}^{V}, f_{3, A}^{A}$ and $f_{3, A}^{\perp}$ still cannot be established.
} 


$$
\begin{aligned}
& i \int d^{4} x e^{i q x}\left\langle 0\left|T\left\{J_{2, \mu}^{3, V}(x), \bar{q}_{1}(0) \gamma_{\nu} \gamma_{5} q_{2}(0)\right\}\right| 0\right\rangle=-T_{3}^{V, \alpha_{1}}\left(q^{2}\right)(q \bar{z})^{3} g_{\mu \nu}^{\perp}+\cdots \\
& i \int d^{4} x e^{i q x}\left\langle 0\left|T\left\{J_{3, \mu}^{3, V}(x), \bar{q}_{1}(0) \gamma_{\nu} \gamma_{5} q_{2}(0)\right\}\right| 0\right\rangle=-T_{3}^{V, \sigma} P_{1}\left(q^{2}\right)(q \bar{z})^{3} g_{\mu \nu}^{\perp}+\cdots
\end{aligned}
$$

where the currents are defined as

$$
\begin{aligned}
& J_{1, \mu}^{3, V}(0)=\bar{z}^{\alpha} \bar{z}^{\beta} \bar{q}_{2}(0) \gamma_{\alpha} g_{s} \widetilde{G}_{\beta \mu}(0) q_{1}(0) \\
& J_{2, \mu}^{3, V}(0)=\bar{z}^{\alpha} \bar{z}^{\beta} \bar{q}_{2}(0) \gamma_{\alpha} g_{s}\left[i D \bar{z} \widetilde{G}_{\beta \mu}(0)\right] q_{1}(0) \\
& J_{3, \mu}^{3, V}(0)=\bar{z}^{\alpha} \bar{z}^{\beta} \bar{q}_{2}(0) \gamma_{\alpha} g_{s} i \bar{z}^{\delta}\left[\widetilde{G}_{\beta \mu}(0) \vec{D}_{\delta}-\overleftarrow{D}_{\delta} \widetilde{G}_{\beta \mu}(0)\right] q_{1}(0)
\end{aligned}
$$

which can couple to the $1^{3} P_{1}$ states as

$$
\begin{aligned}
& \left\langle 0\left|J_{1, \mu}^{3, V}(0)\right| 1^{3} P_{1}(P, \lambda)\right\rangle=-i f_{3,{ }^{3} P_{1}}^{V}(P \bar{z})^{2} \epsilon_{\perp, \mu}^{(\lambda)}+\mathcal{O}\left(\bar{z}_{\mu}\right) \\
& \left\langle 0\left|J_{2, \mu}^{3, V}(0)\right| 1^{3} P_{1}(P, \lambda)\right\rangle=-i f_{3,{ }^{3} P_{1}}^{V}\left\langle\alpha_{g}^{V}\right\rangle(P \bar{z})^{3} \epsilon_{\perp, \mu}^{(\lambda)}+\mathcal{O}\left(\bar{z}_{\mu}\right) \\
& \left\langle 0\left|J_{3, \mu}^{3, V}(0)\right| 1^{3} P_{1}(P, \lambda)\right\rangle=-i f_{3,{ }^{3} P_{1}{ }{ }^{2} P_{1}}^{V}(P \bar{z})^{3} \epsilon_{\perp, \mu}^{(\lambda)}+\mathcal{O}\left(\bar{z}_{\mu}\right),
\end{aligned}
$$

with $\mathcal{O}\left(\bar{z}_{\mu}\right)$ including the twist- 4 correction and the average gluon momentum fraction $\left\langle\alpha_{g}^{V}\right\rangle$ satisfying

$$
\left\langle\alpha_{g}^{V}\right\rangle=\frac{3}{7}+\frac{3}{28} \omega_{3 P_{1}}^{V}
$$

Here and below the ellipses denote terms irrelevant to the present studies. It should be note that $T_{3}^{V} P_{1}, T_{3}^{V, \alpha_{1}}$, and $T_{3}^{V, \sigma}$ receive no contributions from pseudoscalar states.

Assuming the quark-hadron duality, we can obtain the approximate expressions

$$
\begin{aligned}
& \frac{1}{m_{3 P_{1}}^{2}-q^{2}} m_{{ }^{3} P_{1}} f_{{ }^{3} P_{1}} f_{{ }^{3} P_{1}}^{V}=\frac{1}{\pi} \int_{0}^{s_{0}^{s_{0} P_{1}}} d s \frac{\operatorname{Im} T_{3}^{V, \mathrm{OPE}}(s)}{s-q^{2}},
\end{aligned}
$$

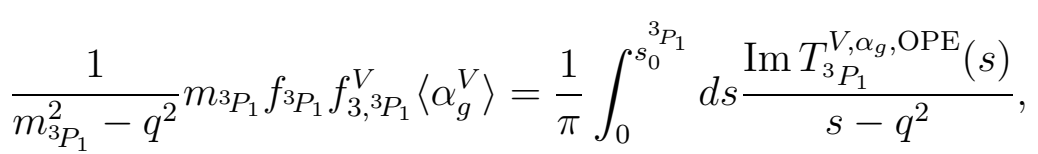

and

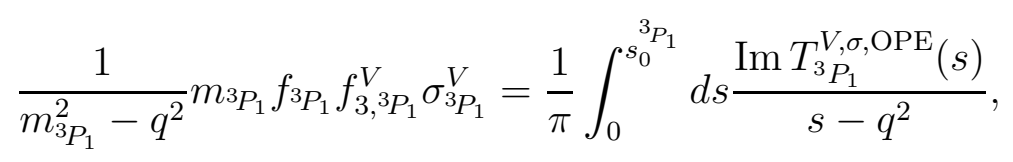

where

$$
\begin{aligned}
T_{3 P_{1}}^{V, \mathrm{OPE}}\left(q^{2}\right) & =-\frac{\alpha_{s}}{144 \pi^{3}} q^{2} \ln \frac{-q^{2}}{\mu^{2}}-\frac{1}{24 \pi q^{2}}\left\langle\alpha_{s} G^{2}\right\rangle+\frac{8 \pi \alpha_{s}}{27 q^{4}}\left(\left\langle\bar{q}_{1} q_{1}\right\rangle^{2}+\left\langle\bar{q}_{2} q_{2}\right\rangle^{2}-3\left\langle\bar{q}_{1} q_{1}\right\rangle\left\langle\bar{q}_{2} q_{2}\right\rangle\right) \\
& +\frac{\alpha_{s}}{36 \pi q^{2}}\left[5\left(m_{1}\left\langle\bar{q}_{1} q_{1}\right\rangle+m_{2}\left\langle\bar{q}_{2} q_{2}\right\rangle\right)+4\left(m_{1}\left\langle\bar{q}_{2} q_{2}\right\rangle+m_{2}\left\langle\bar{q}_{1} q_{1}\right\rangle\right)\left(\ln \frac{-q^{2}}{\mu^{2}}-\frac{17}{6}\right)\right],
\end{aligned}
$$




$$
\begin{aligned}
T_{3 P_{1}}^{V, \alpha_{g}, \mathrm{OPE}}= & -\frac{\alpha_{s}}{120 \pi^{3}} q^{2} \ln \frac{-q^{2}}{\mu^{2}}+\left(0+\mathcal{O}\left(\alpha_{s}\right)\right)\left\langle\alpha_{s} G^{2}\right\rangle-\frac{8 \pi \alpha_{s}}{9 q^{4}}\left\langle\bar{q}_{1} q_{1}\right\rangle\left\langle\bar{q}_{2} q_{2}\right\rangle \\
& +\frac{\alpha_{s}}{12 \pi q^{2}}\left[\frac{1}{2}\left(m_{1}\left\langle\bar{q}_{1} q_{1}\right\rangle+m_{2}\left\langle\bar{q}_{2} q_{2}\right\rangle\right)+\left(m_{1}\left\langle\bar{q}_{2} q_{2}\right\rangle+m_{2}\left\langle\bar{q}_{1} q_{1}\right\rangle\right)\left(\ln \frac{-q^{2}}{\mu^{2}}-\frac{11}{3}\right)\right] .
\end{aligned}
$$

and

$$
\begin{aligned}
T_{3}^{V, \sigma, \mathrm{OPE}}\left(q^{2}\right)= & \frac{-\alpha_{s}}{36 \pi q^{2}}\left[\frac{7}{2}\left(m_{1}\left\langle\bar{q}_{1} q_{1}\right\rangle-m_{2}\left\langle\bar{q}_{2} q_{2}\right\rangle\right)-\left(m_{1}\left\langle\bar{q}_{2} q_{2}\right\rangle-m_{2}\left\langle\bar{q}_{1} q_{1}\right\rangle\right)\left(\ln \frac{-q^{2}}{\mu^{2}}-\frac{11}{3}\right)\right] \\
& +0 \cdot\left\langle\alpha_{s} G^{2}\right\rangle+0 \cdot\left\langle\bar{q}_{1} q_{1}\right\rangle\left\langle\bar{q}_{2} q_{2}\right\rangle+\left(0+\mathcal{O}\left(\alpha_{s}^{2}\right)\right)\left(\left\langle\bar{q}_{1} q_{1}\right\rangle^{2},\left\langle\bar{q}_{2} q_{2}\right\rangle^{2}\right) .
\end{aligned}
$$

Note that $T_{3 P_{1}}^{V}, T_{3}^{V, \alpha}$, and $T_{3 P_{1}}^{V, \sigma}$ receive contributions from ${ }^{1} P_{1}$ states because ${ }^{1} P_{1}$ states have small axial-vector coupling constants due to $m_{q_{1}}-m_{q_{2}} \neq 0$. Consequently, after performing the Borel transform, we obtain the sum rules:

$$
\begin{aligned}
& e^{-m_{3_{P_{1}}}^{2} / M^{2}} m_{{ }^{3} P_{1}} f_{{ }^{3} P_{1}} f_{3,{ }^{3} P_{1}}^{V}+e^{-m_{1}^{2}} / M_{1}^{2} m_{1 P_{1}} f_{{ } P_{1}} a_{0}^{\|, 1^{1} P_{1}} f_{3,{ }^{1} P_{1}}^{V} \lambda_{1}^{V} P_{1} \\
& =\frac{\alpha_{s}}{144 \pi^{3}} \int_{0}^{s_{0}^{s_{0} P_{1}}} s e^{-s / M^{2}} d s+\frac{1}{24 \pi}\left\langle\alpha_{s} G^{2}\right\rangle+\frac{8 \pi \alpha_{s}}{27 M^{2}}\left(\left\langle\bar{q}_{1} q_{1}\right\rangle^{2}+\left\langle\bar{q}_{2} q_{2}\right\rangle^{2}-3\left\langle\bar{q}_{1} q_{1}\right\rangle\left\langle\bar{q}_{2} q_{2}\right\rangle\right) \\
& -\frac{\alpha_{s}}{36 \pi}\left[5\left(m_{1}\left\langle\bar{q}_{1} q_{1}\right\rangle+m_{2}\left\langle\bar{q}_{2} q_{2}\right\rangle\right)\right. \\
& \left.-4\left(m_{1}\left\langle\bar{q}_{2} q_{2}\right\rangle+m_{2}\left\langle\bar{q}_{1} q_{1}\right\rangle\right)\left(\frac{17}{6}+\gamma_{E}+\ln \frac{\mu^{2}}{M^{2}}-\operatorname{Ei}\left(-\frac{s_{0}}{M^{2}}\right)\right)\right]
\end{aligned}
$$

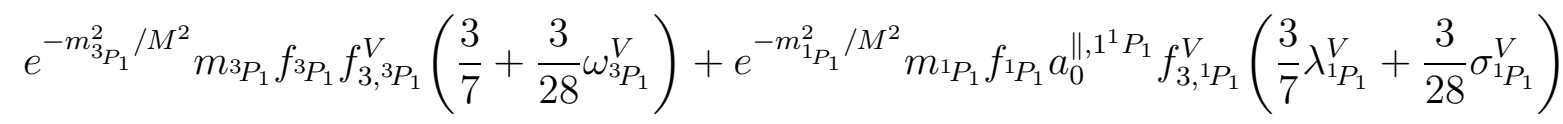

$$
\begin{aligned}
& =\frac{\alpha_{s}}{120 \pi^{3}} \int_{0}^{s_{0}^{s_{0} P_{1}}} s e^{-s / M^{2}} d s-\frac{8 \pi \alpha_{s}}{9 M^{2}}\left\langle\bar{q}_{1} q_{1}\right\rangle\left\langle\bar{q}_{2} q_{2}\right\rangle+\frac{\alpha_{s}}{12 \pi}\left[-\frac{1}{2}\left(m_{1}\left\langle\bar{q}_{1} q_{1}\right\rangle+m_{2}\left\langle\bar{q}_{2} q_{2}\right\rangle\right)\right. \\
& \left.+\left(m_{1}\left\langle\bar{q}_{2} q_{2}\right\rangle+m_{2}\left\langle\bar{q}_{1} q_{1}\right\rangle\right)\left(\frac{11}{3}+\gamma_{E}+\ln \frac{\mu^{2}}{M^{2}}-\operatorname{Ei}\left(-\frac{s_{0}}{M^{2}}\right)\right)\right], \\
& e^{-m_{3_{P_{1}}}^{2} / M^{2}} m_{3 P_{1}} f_{3 P_{1}} f_{3,{ }^{3} P_{1}}^{V} \sigma_{3 P_{1}}^{V}+e^{-m_{1_{P_{1}}}^{2} / M^{2}} m_{{ }^{1} P_{1}} f_{{ }^{1} P_{1}} a_{0}^{\|, 1^{1} P_{1}} f_{3,{ }^{1} P_{1}}^{V} \\
& =\frac{\alpha_{s}}{36 \pi}\left[\frac{7}{2}\left(m_{1}\left\langle\bar{q}_{1} q_{1}\right\rangle-m_{2}\left\langle\bar{q}_{2} q_{2}\right\rangle\right)\right. \\
& \left.+\left(m_{1}\left\langle\bar{q}_{2} q_{2}\right\rangle-m_{2}\left\langle\bar{q}_{1} q_{1}\right\rangle\right)\left(\frac{11}{3}+\gamma_{E}+\ln \frac{\mu^{2}}{M^{2}}-\operatorname{Ei}\left(-\frac{s_{0}}{M^{2}}\right)\right)\right] .
\end{aligned}
$$


As for Eqs. (163) and (164), the contributions, arising from G-parity breaking parameters relevant to twist-3 three-parton LCDAs of the $1^{1} P_{1}$ state, are relatively suppressed by $\mathcal{O}\left(m_{q}^{2}\right)$ and can be negligible. Nevertheless, in Eq. (165), the contribution originating from the $1^{1} P_{1}$ state is of the same order of magnitude as the G-parity breaking parameter relevant to the $1^{3} P_{1}$ state, and should be taken into account. Similar situations occur in Eqs. (178), (179), (180), (193), (194), and (195) in the following subsections 5.1.2 and 5.1.3.

\subsection{2 $f_{3,{ }^{3} P_{1}}^{A}, \lambda_{3 P_{1}}^{A}$, and $\sigma_{3 P_{1}}^{A}$}

The constants $f_{3,{ }^{3} P_{1}}^{A}, \lambda^{A} P_{1}$, and $\sigma_{3 P_{1}}^{A}$ for $1^{3} P_{1}$ mesons can be defined as the following matrix elements

$$
\begin{aligned}
\left\langle 0\left|J_{3, \mu}^{3, A}(0)\right| A(P, \lambda)\right\rangle & =-f_{3,{ }^{3} P_{1}}^{A}(P \bar{z})^{3} \epsilon_{\perp, \mu}^{(\lambda)}+\mathcal{O}\left(\bar{z}_{\mu}\right) \\
\left\langle 0\left|J_{1, \mu}^{3, A}(0)\right| A(P, \lambda)\right\rangle & =-f_{3,{ }^{3} P_{1}}^{A} \lambda^{A} P_{1}(P \bar{z})^{2} \epsilon_{\perp, \mu}^{(\lambda)}+\mathcal{O}\left(\bar{z}_{\mu}\right) \\
\left\langle 0\left|J_{2, \mu}^{3, A}(0)\right| A(P, \lambda)\right\rangle & =-f_{3,{ }^{3} P_{1}}^{A}\left(\frac{3}{7} \lambda^{A} P_{1}+\frac{3}{28} \sigma_{3 P_{1}}^{A}\right)(P \bar{z})^{3} \epsilon_{\perp, \mu}^{(\lambda)}+\mathcal{O}\left(\bar{z}_{\mu}\right),
\end{aligned}
$$

where $\mathcal{O}\left(\bar{z}_{\mu}\right)$ involves the twist-4 correction and the interpolating currents are

$$
\begin{aligned}
& J_{1, \mu}^{3, A}(0)=\bar{z}^{\alpha} \bar{z}^{\beta} \bar{q}_{2}(0) \gamma_{\alpha} \gamma_{5} g_{s} G_{\beta \mu}(0) q_{1}(0) \\
& J_{2, \mu}^{3, A}(0)=\bar{z}^{\alpha} \bar{z}^{\beta} \bar{q}_{2}(0) \gamma_{\alpha} \gamma_{5} g_{s}\left[i D \bar{z} G_{\beta \mu}(0)\right] q_{1}(0) \\
& J_{3, \mu}^{3, A}(0)=\bar{z}^{\alpha} \bar{z}^{\beta} \bar{q}_{2}(0) \gamma_{\alpha} \gamma_{5} g_{s}\left[G_{\beta \mu}(0) i \stackrel{\vec{D}}{z}-i \stackrel{\leftarrow}{D} z G_{\beta \mu}(0)\right] q_{1}(0)
\end{aligned}
$$

Thus, to evaluate $f_{3,{ }^{3} P_{1}}^{A}, \lambda_{3,{ }^{3} P_{1}}^{A}$, and $\sigma_{3,{ }^{3} P_{1}}^{A}$, we consider the non-diagonal correlation functions,

$$
\begin{aligned}
& \int d^{4} x e^{i q x}\left\langle 0\left|T\left\{J_{3, \mu}^{3, A}(x), \bar{q}_{1}(0) \gamma_{\nu} \gamma_{5} q_{2}(0)\right\}\right| 0\right\rangle=T_{3 P_{1}}^{A}\left(q^{2}\right)(q \bar{z})^{3} g_{\mu \nu}^{\perp}+\cdots, \\
& \int d^{4} x e^{i q x}\left\langle 0\left|T\left\{J_{1, \mu}^{3, A}(x), \bar{q}_{1}(0) \gamma_{\nu} \gamma_{5} q_{2}(0)\right\}\right| 0\right\rangle=T_{3 P_{1}}^{A, \lambda}\left(q^{2}\right)(q \bar{z})^{2} g_{\mu \nu}^{\perp}+\cdots, \\
& \int d^{4} x e^{i q x}\left\langle 0\left|T\left\{J_{2, \mu}^{3, A}(x), \bar{q}_{1}(0) \gamma_{\nu} \gamma_{5} q_{2}(0)\right\}\right| 0\right\rangle=T_{3}^{A, \sigma}\left(q^{2}\right)(q \bar{z})^{3} g_{\mu \nu}^{\perp}+\cdots,
\end{aligned}
$$

where we get

$$
\begin{aligned}
T_{3 P_{1}}^{A, \mathrm{OPE}}\left(q^{2}\right)= & -\frac{\alpha_{s}}{1440 \pi^{3}} q^{2} \ln \frac{-q^{2}}{\mu^{2}}-\frac{1}{72 \pi q^{2}}\left\langle\alpha_{s} G^{2}\right\rangle+\frac{\alpha_{s}}{36 \pi q^{2}}\left[\frac{9}{2}\left(m_{1}\left\langle\bar{q}_{1} q_{1}\right\rangle+m_{2}\left\langle\bar{q}_{2} q_{2}\right\rangle\right)\right. \\
& \left.-\left(m_{1}\left\langle\bar{q}_{2} q_{2}\right\rangle+m_{2}\left\langle\bar{q}_{1} q_{1}\right\rangle\right)\left(\ln \frac{-q^{2}}{\mu^{2}}+\frac{1}{3}\right)\right] \\
+ & \left(0+\mathcal{O}\left(\alpha_{s}^{2}\right)\right)\left(\left\langle\bar{q}_{1} q_{1}\right\rangle\left\langle\bar{q}_{2} q_{2}\right\rangle,\left\langle\bar{q}_{1} q_{1}\right\rangle^{2},\left\langle\bar{q}_{2} q_{2}\right\rangle^{2}\right),
\end{aligned}
$$




$$
\begin{aligned}
T_{{ }^{3} P_{1}}^{A, \lambda, \mathrm{OPE}}\left(q^{2}\right)= & \frac{\alpha_{s}}{36 \pi q^{2}}\left[5\left(m_{1}\left\langle\bar{q}_{1} q_{1}\right\rangle-m_{2}\left\langle\bar{q}_{2} q_{2}\right\rangle\right)-4\left(m_{1}\left\langle\bar{q}_{2} q_{2}\right\rangle-m_{2}\left\langle\bar{q}_{1} q_{1}\right\rangle\right)\left(\ln \frac{-q^{2}}{\mu^{2}}-\frac{2}{3}\right)\right] \\
& +0 \cdot\left\langle\alpha_{s} G^{2}\right\rangle-\frac{8 \pi \alpha_{s}}{27 q^{4}}\left(\left\langle\bar{q}_{1} q_{1}\right\rangle^{2}-\left\langle\bar{q}_{2} q_{2}\right\rangle^{2}\right), \\
T_{{ }^{3} P_{1}, \sigma, \mathrm{OPE}}\left(q^{2}\right)= & \frac{\alpha_{s}}{24 \pi q^{2}}\left[\left(m_{1}\left\langle\bar{q}_{1} q_{1}\right\rangle-m_{2}\left\langle\bar{q}_{2} q_{2}\right\rangle\right)-2\left(m_{1}\left\langle\bar{q}_{2} q_{2}\right\rangle-m_{2}\left\langle\bar{q}_{1} q_{1}\right\rangle\right)\left(\ln \frac{-q^{2}}{\mu^{2}}-1\right)\right] \\
& +0 \cdot\left\langle\alpha_{s} G^{2}\right\rangle+0 \cdot\left\langle\bar{q}_{1} q_{1}\right\rangle\left\langle\bar{q}_{2} q_{2}\right\rangle+\left(0+\mathcal{O}\left(\alpha_{s}^{2}\right)\right)\left(\left\langle\bar{q}_{1} q_{1}\right\rangle^{2},\left\langle\bar{q}_{2} q_{2}\right\rangle^{2}\right) .
\end{aligned}
$$

From the above OPE results, it follows the sum rules:

$$
\begin{aligned}
& e^{-m_{{ }^{2} P_{1}}^{2} / M^{2}} m_{3 P_{1}} f_{3} P_{1} f_{3,{ }^{3} P_{1}}^{A}+e^{-m_{1}^{2}{ }_{P_{1}} / M^{2}} m_{1 P_{1}} f_{1 P_{1}} a_{0}^{\|, 1^{1} P_{1}} f_{3,{ }^{1} P_{1}}^{A} \sigma_{1}^{A} P_{1} \\
& =\frac{\alpha_{s}}{1440 \pi^{3}} \int_{0}^{s_{0}^{s_{0}} P_{1}} s e^{-s / M^{2}} d s+\frac{1}{72 \pi}\left\langle\alpha_{s} G^{2}\right\rangle+\frac{\alpha_{s}}{36 \pi}\left[-\frac{9}{2}\left(m_{1}\left\langle\bar{q}_{1} q_{1}\right\rangle+m_{2}\left\langle\bar{q}_{2} q_{2}\right\rangle\right)\right. \\
& \left.+\left(m_{1}\left\langle\bar{q}_{2} q_{2}\right\rangle+m_{2}\left\langle\bar{q}_{1} q_{1}\right\rangle\right)\left(\frac{1}{3}-\gamma_{E}-\ln \frac{\mu^{2}}{M^{2}}+\operatorname{Ei}\left(-\frac{s_{0}}{M^{2}}\right)\right)\right], \\
& e^{-m_{3_{P_{1}}}^{2} / M^{2}} m_{3 P_{1}} f_{{ }^{3} P_{1}} f_{3,{ }^{3} P_{1}}^{A} \lambda_{3 P_{1}}^{A}+e^{-m_{{ }_{1} P_{1}}^{2} / M^{2}} m_{1 P_{1}} f_{1} P_{1} a_{0}^{\|, 1^{1} P_{1}} f_{3,{ }^{\prime} P_{1}}^{A} \\
& =-\frac{8 \pi \alpha_{s}}{27 M^{2}}\left(\left\langle\bar{q}_{1} q_{1}\right\rangle^{2}-\left\langle\bar{q}_{2} q_{2}\right\rangle^{2}\right)+\frac{\alpha_{s}}{36 \pi}\left[-5\left(m_{1}\left\langle\bar{q}_{1} q_{1}\right\rangle-m_{2}\left\langle\bar{q}_{2} q_{2}\right\rangle\right)\right. \\
& \left.+4\left(m_{1}\left\langle\bar{q}_{2} q_{2}\right\rangle-m_{2}\left\langle\bar{q}_{1} q_{1}\right\rangle\right)\left(-\frac{2}{3}-\gamma_{E}-\ln \frac{\mu^{2}}{M^{2}}+\operatorname{Ei}\left(-\frac{s_{0}}{M^{2}}\right)\right)\right]
\end{aligned}
$$

and

$$
\begin{aligned}
& e^{-m_{3_{P_{1}}}^{2} / M^{2}} m_{3 P_{1}} f_{3} P_{1} f_{3,{ }^{3} P_{1}}^{A}\left(\frac{3}{7} \lambda^{A} P_{1}+\frac{3}{28} \sigma_{3 P_{1}}^{A}\right)+e^{-m_{1}^{2}{ }_{P_{1}} / M^{2}} m_{{ }_{1} P_{1}} f_{1 P_{1}} a_{0}^{\|, 1^{1} P_{1}} f_{3,{ }^{\prime} P_{1}}^{A}\left(\frac{3}{7}+\frac{3}{28} \omega_{1 P_{1}}^{A}\right) \\
& =\frac{\alpha_{s}}{24 \pi}\left[-\left(m_{1}\left\langle\bar{q}_{1} q_{1}\right\rangle-m_{2}\left\langle\bar{q}_{2} q_{2}\right\rangle\right)\right. \\
& \left.+2\left(m_{1}\left\langle\bar{q}_{2} q_{2}\right\rangle-m_{2}\left\langle\bar{q}_{1} q_{1}\right\rangle\right)\left(-1-\gamma_{E}-\ln \frac{\mu^{2}}{M^{2}}+\operatorname{Ei}\left(-\frac{s_{0}}{M^{2}}\right)\right)\right] .
\end{aligned}
$$

\subsection{3 $f_{3,{ }^{3} P_{1}}^{\perp}, \omega^{\frac{\perp}{3} P_{1}}$ and $\sigma^{\perp} P_{1}$}

To evaluate the coupling constants $f_{3,{ }^{3} P_{1}}^{\perp}, \omega^{\perp} P_{1}$ and $\sigma_{{ }^{3} P_{1}}^{\perp}$ of $1^{3} P_{1}$ states, we first consider the following non-diagonal correlation functions,

$$
\begin{gathered}
i \int d^{4} x e^{i q x}\left\langle 0\left|T\left\{J_{1}^{3, \perp}(x), \bar{q}_{1}(0) \gamma_{\mu} \gamma_{5} q_{2}(0)\right\}\right| 0\right\rangle=T_{3 P_{1}}^{\perp}\left(q^{2}\right)(q \bar{z}) \bar{z}_{\mu}+\cdots, \\
i \int d^{4} x e^{i q x}\left\langle 0\left|T\left\{J_{2}^{3, \perp}(x), \bar{q}_{1}(0) \gamma_{\mu} \gamma_{5} q_{2}(0)\right\}\right| 0\right\rangle=T_{3 P_{1}}^{\perp, \alpha_{g}}\left(q^{2}\right)(q \bar{z})^{2} \bar{z}_{\mu}+\cdots,
\end{gathered}
$$




$$
i \int d^{4} x e^{i q x}\left\langle 0\left|T\left\{J_{3}^{3, \perp}(x), \bar{q}_{1}(0) \gamma_{\mu} \gamma_{5} q_{2}(0)\right\}\right| 0\right\rangle=T_{3 P_{1}}^{\perp, \sigma}\left(q^{2}\right)(q \bar{z})^{2} \bar{z}_{\mu}+\cdots,
$$

where

$$
\begin{aligned}
& J_{1}^{3, \perp}(0)=\bar{q}_{2}(0) \bar{z}^{\beta} \bar{z}_{\lambda} \sigma_{\alpha \beta} \gamma_{5} g_{s} G^{\lambda \alpha}(0) q_{1}(0) \\
& J_{2}^{3, \perp}(0)=\bar{q}_{2}(0) \bar{z}^{\beta} \bar{z}_{\lambda} \sigma_{\alpha \beta} \gamma_{5} g_{s}\left[i D \bar{z} G^{\lambda \alpha}(0)\right] q_{1}(0) \\
& J_{3}^{3, \perp}(0)=\bar{q}_{2}(0) \bar{z}^{\beta} \bar{z}_{\lambda} \sigma_{\alpha \beta} \gamma_{5} g_{s} i \bar{z}^{\delta}\left[G^{\lambda \alpha}(0) \vec{D}_{\delta}-\overleftarrow{D}_{\delta} G^{\lambda \alpha}(0)\right] q_{1}(0)
\end{aligned}
$$

To including the light quark masses consistently to the $\mathcal{O}\left(\alpha_{s}\right)$, the currents needs to be replaced by the renormalized ones as

$$
\begin{aligned}
& J_{1}^{3, \perp} \longrightarrow \bar{J}_{1}^{3, \perp}=J_{1}^{3, \perp}+\frac{\alpha_{s}}{4 \pi} \frac{4}{9 \hat{\varepsilon}}\left(m_{1}+m_{2}\right) \bar{z}^{\alpha} i D \cdot \bar{z}\left(\bar{q}_{2} \not z \gamma_{5} q_{1}\right), \\
& J_{2}^{3, \perp} \longrightarrow \bar{J}_{2}^{3, \perp}=J_{2}^{3, \perp}+\frac{\alpha_{s}}{4 \pi} \frac{1}{5 \hat{\varepsilon}}\left(m_{1}+m_{2}\right) \bar{z}^{\alpha}(i D \cdot \bar{z})^{2}\left(\bar{q}_{2} \not z \gamma_{5} q_{1}\right), \\
& J_{3}^{3, \perp} \longrightarrow \bar{J}_{3}^{3, \perp}=J_{3}^{3, \perp}-\frac{\alpha_{s}}{4 \pi} \frac{1}{9 \hat{\varepsilon}}\left(m_{1}-m_{2}\right) \bar{z}^{\alpha}(i D \cdot \bar{z})^{2}\left(\bar{q}_{2} \not \not z \gamma_{5} q_{1}\right),
\end{aligned}
$$

where

$$
\frac{1}{\hat{\varepsilon}}=\frac{1}{\varepsilon}+\gamma_{E}-\ln 4 \pi
$$

and $d$ (dimension) $=4+2 \varepsilon$. As adopted in all the calculations of this paper, the modified minimal substraction scheme $(\overline{\mathrm{MS}})$ is used to regularize the divergent integrals. From the technical point of view, the reason that we have to take into account the mixings of $J_{i}^{3, \perp}$ and twist-2 operators is because we need to remove the nonphysical $\ln \left(-q^{2} / \mu^{2}\right) / \hat{\varepsilon}$ terms in the calculation. Physically speaking, $f_{3,{ }_{3} P_{1}}^{\perp}$ and $f_{3,{ }_{3} P_{1}}^{\perp} \omega_{3 P_{1}}^{\perp}$ mix with $f_{3} P_{1}\left(m_{q_{1}}+m_{q_{2}}\right)$, while $f_{3,3 P_{1}}^{\perp} \sigma_{3 P_{1}}^{\perp}$ mixes with $f_{P_{1}}\left(m_{q_{1}}-m_{q_{2}}\right)$. For a massive quark, the above mixings were studied in Ref. [20] by using the light-ray-operator technique [41]. According to their results, (i) $f_{3,{ }^{\prime} P_{1}}^{\perp}$ can mix with $\left(m_{q_{1}}+m_{q_{2}}\right) f_{{ }^{3} P_{1}}$ and $\left(m_{q_{1}}+m_{q_{2}}\right) f_{3 P_{1}} a_{1}^{\|,{ }^{3} P_{1}}$, (ii) $f_{3,{ }^{3} P_{1}}^{\perp} \omega_{3 P_{1}}^{\perp}$ with $\left(m_{q_{1}}+m_{q_{2}}\right) f_{3} P_{1}$ and $\left(m_{q_{1}}+m_{q_{2}}\right) f^{3} P_{1} a_{1}^{\|,{ }^{3} P_{1}}$, and $\left(m_{q_{1}}+m_{q_{2}}\right) f_{3 P_{1}} a_{2}^{\|,{ }^{3} P_{1}}$, and (iii) $f_{3,{ }^{3} P_{1}}^{\perp} \sigma_{3 P_{1}}^{\perp}$ with $\left(m_{q_{1}}-m_{q_{2}}\right) f^{3} P_{1}$ and $\left(m_{q_{1}}-m_{q_{2}}\right) f_{3_{3} P_{1}} a_{1}^{\|,{ }^{3} P_{1}}$, and $\left(m_{q_{1}}-m_{q_{2}}\right) f_{3 P_{1}} a_{2}^{\|,{ }^{3} P_{1}}$. Neglecting the corrections arising from $a_{1}^{\|,{ }^{3} P_{1}}$ and $a_{2}^{\|,{ }^{3} P_{1}}$, our results basically agree with those given in Ref. [20] except a sign difference for $J_{3}^{3, \perp}$. To LO approximation, the complete RG evolutions of the relevant parameters are collected in Appendix C.

$T_{3 P_{1}}^{\perp}\left(q^{2}\right), T_{3_{1}}^{\perp, \alpha_{g}}$, and $T_{3 P_{1}}^{\perp, \sigma}$ are relevant for the present consideration and the OPE results are given by

$$
\begin{aligned}
T_{3 P_{1}}^{\perp, \mathrm{OPE}}\left(q^{2}\right)= & \left(m_{1}+m_{2}\right) \frac{\alpha_{s}}{144 \pi^{3}} q^{2} \ln \frac{-q^{2}}{\mu^{2}}\left[5-2 \ln \frac{-q^{2}}{\mu^{2}}\right]+\left(0+\mathcal{O}\left(\alpha_{s}^{2}\right)\right)\left(\left\langle\bar{q}_{1} q_{1}\right\rangle+\left\langle\bar{q}_{2} q_{2}\right\rangle\right) \\
& -\frac{1}{108 q^{2}}\left(\ln \frac{-q^{2}}{\mu^{2}}-\frac{547}{96}\right) \frac{\alpha_{s}}{\pi}\left(\left\langle\bar{q}_{1} g_{s} \sigma G q_{1}\right\rangle+\left\langle\bar{q}_{2} g_{s} \sigma G q_{2}\right\rangle\right) \\
& -\frac{\pi}{9 q^{4}}\left\langle\alpha_{s} G^{2}\right\rangle\left(\left\langle\bar{q}_{1} q_{1}\right\rangle+\left\langle\bar{q}_{2} q_{2}\right\rangle\right),
\end{aligned}
$$




$$
\begin{aligned}
& T_{3 P_{1}}^{\perp, \alpha_{g}, \mathrm{OPE}}\left(q^{2}\right)=\left(m_{1}+m_{2}\right) \frac{\alpha_{s}}{320 \pi^{3}} q^{2} \ln \frac{-q^{2}}{\mu^{2}}\left[\frac{83}{10}-2 \ln \frac{-q^{2}}{\mu^{2}}\right]+\left(0+\mathcal{O}\left(\alpha_{s}^{2}\right)\right)\left(\left\langle\bar{q}_{1} q_{1}\right\rangle+\left\langle\bar{q}_{2} q_{2}\right\rangle\right) \\
& -\frac{1}{216 q^{2}}\left(\ln \frac{-q^{2}}{\mu^{2}}-\frac{823}{96}\right) \frac{\alpha_{s}}{\pi}\left(\left\langle\bar{q}_{1} g_{s} \sigma G q_{1}\right\rangle+\left\langle\bar{q}_{2} g_{s} \sigma G q_{2}\right\rangle\right) \\
& +\left(0+\mathcal{O}\left(\alpha_{s}\right)\right)\left\langle\alpha_{s} G^{2}\right\rangle\left(\left\langle\bar{q}_{1} q_{1}\right\rangle+\left\langle\bar{q}_{2} q_{2}\right\rangle\right), \\
& T_{3 P_{1}}^{\perp, \sigma, \mathrm{OPE}}\left(q^{2}\right)=\left(m_{1}-m_{2}\right) \frac{\alpha_{s}}{576 \pi^{3}} q^{2} \ln \frac{-q^{2}}{\mu^{2}}\left[\frac{47}{5}-2 \ln \frac{-q^{2}}{\mu^{2}}\right] \\
& -\frac{\alpha_{s}}{6 \pi}\left(\ln \frac{-q^{2}}{\mu^{2}}+\text { const. }\right)\left(\left\langle\bar{q}_{1} q_{1}\right\rangle-\left\langle\bar{q}_{2} q_{2}\right\rangle\right) \\
& +\frac{1}{216 q^{2}}\left(\ln \frac{-q^{2}}{\mu^{2}}-\frac{847}{96}\right) \frac{\alpha_{s}}{\pi}\left(\left\langle\bar{q}_{1} g_{s} \sigma G q_{1}\right\rangle-\left\langle\bar{q}_{2} g_{s} \sigma G q_{2}\right\rangle\right) \\
& +\frac{\pi}{9 q^{4}}\left\langle\alpha_{s} G^{2}\right\rangle\left(\left\langle\bar{q}_{1} q_{1}\right\rangle-\left\langle\bar{q}_{2} q_{2}\right\rangle\right) \text {. }
\end{aligned}
$$

For the coefficient of the quark-gluon condensate in the above equations, the logarithmic factor arises from the contribution of the diagrams that contains a loop connecting a quark propagator, where the quark propagator (which does not belong to the part of the loop) emits a soft gluon into the condensate. Note that there is no infrared pole (IR) in the calculations of the present work due to the fact that the off-shell external momentum $-q^{2}<0$ regularize the IR singularity in QCD sum rule approach. (It is interesting to note that the infrared sensitive terms $\sim \ln \left(-q^{2} / m_{q}^{2}\right)$ may appear as considering the order up to $m_{q}^{2}$. However, they can be absorbed into the condensate [42].)

The sum rules for $f_{3,{ }^{3} P_{1}}^{\perp}, \omega^{\frac{1}{3} P_{1}}$ and $\sigma_{3 P_{1}}^{\perp}$ therefore read

$$
\begin{aligned}
& e^{-m_{{ }^{2} P_{1}}^{2} / M^{2}} m_{3}^{2} P_{1} f_{3} P_{1} f_{3,{ }^{3} P_{1}}^{\perp}+e^{-m_{1}^{2} P_{1} / M^{2}} m_{{ }_{1} P_{1}}^{2} f_{{ } P_{1}} a_{0}^{\|, 1^{1} P_{1}} f_{3,{ }^{1} P_{1}}^{\perp} \lambda_{1}^{\perp} P_{1} \\
& =\left(m_{1}+m_{2}\right) \frac{\alpha_{s}}{144 \pi^{3}} \int_{0}^{s_{0}^{3} P_{1}} s\left(-5+4 \ln \frac{s}{\mu^{2}}\right) e^{-s / M^{2}} d s \\
& -\frac{\alpha_{s}}{108 \pi}\left[\frac{547}{96}+\left(\gamma_{E}+\ln \frac{\mu^{2}}{M^{2}}-\operatorname{Ei}\left(-\frac{s_{0}}{M^{2}}\right)\right]\left(\left\langle\bar{q}_{1} g_{s} \sigma G q_{1}\right\rangle+\left\langle\bar{q}_{2} g_{s} \sigma G q_{2}\right\rangle\right)\right. \\
& -\frac{\pi}{9 M^{2}}\left\langle\alpha_{s} G^{2}\right\rangle\left(\left\langle\bar{q}_{1} q_{1}\right\rangle+\left\langle\bar{q}_{2} q_{2}\right\rangle\right)
\end{aligned}
$$

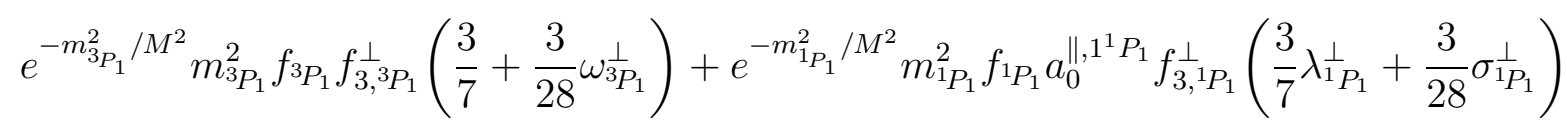

$$
\begin{aligned}
& =\left(m_{1}+m_{2}\right) \frac{\alpha_{s}}{320 \pi^{3}} \int_{0}^{s_{0}^{s_{0}} P_{1}} s\left(-\frac{83}{10}+4 \ln \frac{s}{\mu^{2}}\right) e^{-s / M^{2}} d s \\
& -\frac{\alpha_{s}}{216 \pi}\left[\frac{823}{96}+\left(\gamma_{E}+\ln \frac{\mu^{2}}{M^{2}}-\operatorname{Ei}\left(-\frac{s_{0}}{M^{2}}\right)\right)\right]\left(\left\langle\bar{q}_{1} g_{s} \sigma G q_{1}\right\rangle+\left\langle\bar{q}_{2} g_{s} \sigma G q_{2}\right\rangle\right),
\end{aligned}
$$


and

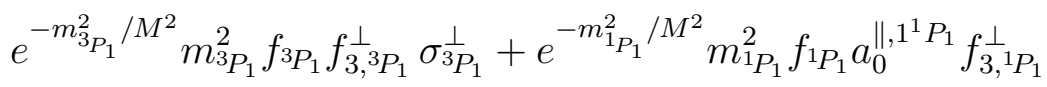

$$
\begin{aligned}
& =\left(m_{1}-m_{2}\right) \frac{\alpha_{s}}{576 \pi^{3}} \int_{0}^{s_{0}{ }^{3} P_{1}} s\left(-\frac{47}{5}+4 \ln \frac{s}{\mu^{2}}\right) e^{-s / M^{2}} d s \\
& +\frac{\alpha_{s}}{6 \pi}\left(\left\langle\bar{q}_{1} q_{1}\right\rangle-\left\langle\bar{q}_{2} q_{2}\right\rangle\right) \int_{0}^{s_{0}^{s_{0} P_{1}}} e^{-s / M^{2}} d s \\
& +\frac{\alpha_{s}}{216 \pi}\left[\frac{847}{96}+\left(\gamma_{E}+\ln \frac{\mu^{2}}{M^{2}}-\operatorname{Ei}\left(-\frac{s_{0}}{M^{2}}\right)\right]\left(\left\langle\bar{q}_{1} g_{s} \sigma G q_{1}\right\rangle-\left\langle\bar{q}_{2} g_{s} \sigma G q_{2}\right\rangle\right)\right. \\
& +\frac{\pi}{9 M^{2}}\left\langle\alpha_{s} G^{2}\right\rangle\left(\left\langle\bar{q}_{1} q_{1}\right\rangle-\left\langle\bar{q}_{2} q_{2}\right\rangle\right) \text {. }
\end{aligned}
$$

Unfortunately, one can read from Eq. (190) or Eq. (193) that for the $f_{3,{ }^{3} P_{1}}^{\perp}$ sum rule the term of dimension-3 in OPE series vanishes in $\mathcal{O}\left(\alpha_{s}\right)$, while the terms of dimensions-5 and -7 are comparable in magnitude but with the opposite signs. As a result, the OPE series does not show any convergent behavior.

Therefore, to evaluate $f_{3,{ }^{3} P_{1}}^{\perp}$, we further consider the following diagonal correlation function,

$$
i \int d^{4} x e^{i q x}\left\langle 0\left|T\left\{J_{\perp}^{3,{ }^{3} P_{1}}(x), J_{\perp}^{3,{ }^{3} P_{1}}(0)\right\}\right| 0\right\rangle=-\widetilde{T}^{\perp}\left(q^{2}\right)(q \bar{z})^{4}
$$

However, at the hadronic level, the lowest-lying resonance for the above correlation function is the pseudoscalar meson (the $0^{-}$state). To subtract the contribution arising from the lowest pseudoscalar meson, we study the following non-diagonal correlation function:

$$
i \int d^{4} x e^{i q x}\left\langle 0\left|T\left\{J_{\omega_{\perp}^{3,}{ }^{3} P_{1}}(x), q_{1}(0) \gamma_{5} q_{2}(0)\right\}\right| 0\right\rangle=-\widetilde{T}^{\prime \perp}\left(q^{2}\right)(q \bar{z})^{5},
$$

where

$$
z^{\beta} z^{\mu}\left\langle 0\left|q_{2} \sigma_{\alpha \beta} \gamma_{5} g_{s} G_{\mu}{ }^{\alpha} q_{1}\right| P S(P)\right\rangle=2 i f_{3 P S}(P z)^{2}
$$

with "PS $\equiv$ the lowest-lying pseudoscalar meson". Only the pseudoscalar mesons contribute to the above non-diagonal correlation function. $\widetilde{T}^{\perp}\left(q^{2}\right)$ and $\widetilde{T}^{\prime}\left(q^{2}\right)$ have been calculated in Ref. [20] for studying $f_{3 K}$.

Using the results given in Ref. [20, the corresponding sum rules are

$$
\begin{aligned}
4 e^{-m_{P S}^{2} / M^{2}} & f_{3 P S}^{2}+e^{-m_{3_{P_{1}}}^{2} / M^{2}}\left(f_{3,{ }^{3} P_{1}}^{\perp}\right)^{2}=\frac{\alpha_{s}}{360 \pi^{3}} \int_{0}^{{ }^{s^{3} P_{1}}} s e^{-s / M^{2}} d s+\frac{89}{5184} \frac{\alpha_{s}}{\pi^{2}}\left\langle\alpha_{s} G^{2}\right\rangle \\
+ & \frac{\alpha_{s}}{18 \pi}\left(m_{q_{1}}\left\langle\bar{q}_{1} q_{1}\right\rangle+m_{q_{2}}\left\langle\bar{q}_{2} q_{2}\right\rangle\right)-\frac{\alpha_{s}}{108 \pi} \frac{1}{M^{2}}\left(m_{q_{1}}\left\langle\bar{q}_{1} g_{s} \sigma G q_{1}\right\rangle+m_{q_{2}}\left\langle\bar{q}_{2} g_{s} \sigma G q_{2}\right\rangle\right) \\
+ & \frac{71}{729} \frac{\alpha_{s}^{2}}{M^{2}}\left(\left\langle\bar{q}_{1} q_{1}\right\rangle^{2}+\left\langle\bar{q}_{2} q_{2}\right\rangle^{2}\right)+\frac{32}{81} \frac{\alpha_{s}^{2}}{M^{2}}\left\langle\bar{q}_{1} q_{1}\right\rangle\left\langle\bar{q}_{2} q_{2}\right\rangle
\end{aligned}
$$


and

$$
\begin{aligned}
2 e^{-m_{P S}^{2} / M^{2}} & f_{3 P S} \frac{f_{P S} m_{P S}^{2}}{m_{q_{1}}+m_{q_{2}}}=\frac{\alpha_{s}}{72 \pi^{3}} \int_{0}^{s_{0}^{P S}} s e^{-s / M^{2}} d s+\frac{1}{12 \pi}\left\langle\alpha_{s} G^{2}\right\rangle \\
& -\frac{\alpha_{s}}{9 \pi}\left(m_{q_{1}}\left\langle\bar{q}_{1} q_{1}\right\rangle+m_{q_{2}}\left\langle\bar{q}_{2} q_{2}\right\rangle\right) \\
& -\frac{2 \alpha_{s}}{9 \pi}\left(m_{q_{1}}\left\langle\bar{q}_{2} q_{2}\right\rangle+m_{q_{2}}\left\langle\bar{q}_{1} q_{1}\right\rangle\right)\left(\frac{8}{3}+\gamma_{E}+\ln \frac{\mu^{2}}{M^{2}}-\operatorname{Ei}\left(-\frac{s_{0}}{M^{2}}\right)\right) \\
& -\frac{1}{6 M^{2}}\left(m_{q_{1}}\left\langle\bar{q}_{2} g_{s} \sigma G q_{2}\right\rangle+m_{q_{2}}\left\langle\bar{q}_{1} g_{s} \sigma G q_{1}\right\rangle\right) \\
& +\frac{16}{27} \frac{\pi \alpha_{s}}{M^{2}}\left(\left\langle\bar{q}_{1} q_{1}\right\rangle^{2}+\left\langle\bar{q}_{2} q_{2}\right\rangle^{2}\right)+\frac{16}{9} \frac{\pi \alpha_{s}}{M^{2}}\left\langle\bar{q}_{1} q_{1}\right\rangle\left\langle\bar{q}_{2} q_{2}\right\rangle .
\end{aligned}
$$

In calculating the $f_{3,{ }^{3} P_{1}}^{\perp}$ sum rule, we substitute $f_{3 P S}$ by using the expression given in Eq. (200). Although the sign of $f_{3,{ }^{3} P_{1}}^{\perp}$ compared with $f_{{ }^{3} P_{1}}$ cannot be determined from Eq. (199), there are two indications that the sign of $f_{3,{ }_{3} P_{1}}^{\perp}$ should be negative. One is that at the large $M^{2}$ limit the result of Eq. (193) implies the negative $f_{3,{ }^{3} P_{1}}^{\perp}$ although the sum rule cannot offer its reliable magnitude. The other one is that only the negative $f_{3,{ }^{3} P_{1}}^{\perp}$ can result in a physical value for $\left\langle\alpha_{g}^{\perp}\right\rangle$, the average gluon momentum fraction in a $1^{3} P_{1}$ meson, which should satisfy

$$
0 \leq\left\langle\alpha_{g}^{\perp}\right\rangle=\frac{3}{7}+\frac{3}{28} \omega_{3 P_{1}}^{\perp} \leq 1
$$

Note that, as for $\omega_{3 P_{1}}^{\perp}$ sum rule (Eq. (194)) which is dominated by the quark-gluon condensate, because the contribution $\mathcal{O}\left(\alpha_{s}\right)$ of the dimension-7 term, $\sim\left\langle\alpha_{s} G^{2}\right\rangle\left(\left\langle\bar{q}_{1} q_{1}\right\rangle+\left\langle\bar{q}_{2} q_{2}\right\rangle\right)$, is absent, we thus do not further study a different correlation function.

\subsection{Axial-vector mesons with quantum number $1^{1} P_{1}$}

5.2.1 $f_{3,{ }^{1} P_{1}}^{V}, \lambda_{1}^{V}$, and $\sigma_{P_{1}}^{V}$

The coupling constants $f_{3,{ }_{1} P_{1}}^{V}, \lambda_{{ }_{1} P_{1}}^{V}$, and $\sigma_{{ }^{1} P_{1}}^{V}$ can be obtained through the following matrix element

$$
\begin{aligned}
\left\langle 0\left|J_{3, \mu}^{3, V}(0)\right| 1^{1} P_{1}(P, \lambda)\right\rangle & =-i f_{3,{ }^{1} P_{1}}^{V}(P \bar{z})^{3} \epsilon_{\perp, \mu}^{(\lambda)}+\mathcal{O}\left(\bar{z}_{\mu}\right), \\
\left\langle 0\left|J_{1, \mu}^{3, V}(0)\right| 1^{1} P_{1}(P, \lambda)\right\rangle & =-i f_{3,{ }^{1} P_{1}}^{V} \lambda^{V} P_{1}(P \bar{z})^{2} \epsilon_{\perp, \mu}^{(\lambda)}+\mathcal{O}\left(\bar{z}_{\mu}\right), \\
\left\langle 0\left|J_{2, \mu}^{3, V}(0)\right| 1^{1} P_{1}(P, \lambda)\right\rangle & =-i f_{3,{ }^{1} P_{1}}^{V}\left(\frac{3}{7} \lambda^{V}{ }_{P_{1}}+\frac{3}{28} \sigma^{V} P_{1}\right)(P \bar{z})^{3} \epsilon_{\perp, \mu}^{(\lambda)}+\mathcal{O}\left(\bar{z}_{\mu}\right),
\end{aligned}
$$

where the currents have been defined in Eqs. (150), (151), and (152). To evaluate $f_{3,{ }_{1}{ }^{1}}^{V}$, $\lambda_{{ }^{\prime}}^{V}$, and $\sigma_{{ }^{1}}^{V}$, we consider the following "non-diagonal" correlation functions,

$$
\int d^{4} x e^{i q x}\left\langle 0\left|T\left\{J_{3, \mu}^{3, V}(x), \bar{q}_{1}(0) \bar{z}^{\lambda} \sigma_{\nu \lambda} \gamma_{5} q_{2}(0)\right\}\right| 0\right\rangle=T_{P_{1}}^{V}\left(q^{2}\right)(q \bar{z})^{4} g_{\mu \nu}^{\perp}+\cdots,
$$




$$
\begin{aligned}
& \int d^{4} x e^{i q x}\left\langle 0\left|T\left\{J_{1, \mu}^{3, V}(x), \bar{q}_{1}(0) \bar{z}^{\lambda} \sigma_{\nu \lambda} \gamma_{5} q_{2}(0)\right\}\right| 0\right\rangle=T_{P_{1}}^{V, \lambda}\left(q^{2}\right)(q \bar{z})^{3} g_{\mu \nu}^{\perp}+\cdots, \\
& \int d^{4} x e^{i q x}\left\langle 0\left|T\left\{J_{2, \mu}^{3, V}(x), \bar{q}_{1}(0) \bar{z}^{\lambda} \sigma_{\nu \lambda} \gamma_{5} q_{2}(0)\right\}\right| 0\right\rangle=T_{P_{1}}^{V, \sigma}\left(q^{2}\right)(q \bar{z})^{4} g_{\mu \nu}^{\perp}+\cdots .
\end{aligned}
$$

Concerning the light quark masse corrections to the correlation functions, we have to replace the currents by the renormalized ones:

$$
\begin{aligned}
J_{3, \mu}^{3, V} \longrightarrow \bar{J}_{3, \mu}^{3, V} & =J_{3, \mu}^{3, V}+\frac{\alpha_{s}}{4 \pi} \frac{1}{18 \hat{\varepsilon}}\left(m_{1}+m_{2}\right) \bar{z}^{\alpha} i(i D \cdot \bar{z})^{2}\left(\bar{q}_{2} \sigma_{\alpha \mu} \gamma_{5} q_{1}\right), \\
J_{1, \mu}^{3, V} \longrightarrow \bar{J}_{1, \mu}^{3, V} & =J_{1, \mu}^{3, V}-\frac{\alpha_{s}}{4 \pi} \frac{2}{9 \hat{\varepsilon}}\left(m_{1}-m_{2}\right) \bar{z}^{\alpha} i(i D \cdot \bar{z})\left(\bar{q}_{2} \sigma_{\alpha \mu} \gamma_{5} q_{1}\right), \\
J_{2, \mu}^{3, V} \longrightarrow \bar{J}_{2, \mu}^{3, V} & =J_{2, \mu}^{3, V}-\frac{\alpha_{s}}{4 \pi} \frac{1}{10 \hat{\varepsilon}}\left(m_{1}-m_{2}\right) \bar{z}^{\alpha} i(i D \cdot \bar{z})^{2}\left(\bar{q}_{2} \sigma_{\alpha \mu} \gamma_{5} q_{1}\right) .
\end{aligned}
$$

The above mixings lead to that $f_{3,{ }^{1} P_{1}}^{V}$ mixes with $f_{{ }_{1} P_{1}}^{\perp}\left(m_{q_{1}}+m_{q_{2}}\right)$, while $f_{3,{ }_{1} P_{1}}^{V} \lambda_{{ }_{1} P_{1}}^{\perp}$ and $f_{3,{ }^{1} P_{1}}^{V} \sigma_{{ }^{1} P_{1}}^{\perp}$ mix with $f_{1_{P_{1}}}^{\perp}\left(m_{q_{1}}-m_{q_{2}}\right)$. We did not find any explicit result in the literature that can be used to compare with the present calculations. However, in analogy to the discussion after Eq. (189), the relevant parameters can mix in addition with $f_{{ }_{1} P_{1}}^{\perp}\left(m_{q_{1}} \pm m_{q_{2}}\right) a_{1}^{\perp,}{ }^{1} P_{1}$ and $f_{{ }_{1} P_{1}}^{\perp}\left(m_{q_{1}} \pm m_{q_{2}}\right) a_{2}^{\perp,}{ }^{1} P_{1}$, where the upper sign corresponds to the G-parity conserving parameter $f_{3,{ }_{1} P_{1}}^{V}$ and the lower sign to the G-parity violating parameters for which $f_{3,{ }_{1} P_{1}}^{V} \lambda_{{ }_{1} P_{1}}^{\perp}$ does not mix with $f_{{ }^{1} P_{1}}^{\perp}\left(m_{q_{1}}-m_{q_{2}}\right) a_{2}^{\perp,}{ }^{1} P_{1}$. We have neglected the RG-corrections due to $\left(m_{q_{1}} \pm m_{q_{2}}\right) a_{1}^{\perp,{ }^{1} P_{1}}$ and $\left(m_{q_{1}} \pm m_{q_{2}}\right) a_{2}^{\perp,}{ }^{1} P_{1}$ in the present calculations. Considering the mass corrections in the RG equations, the scale dependence of the parameters relevant to the twist-three three-parton LCDAs is summarized in Appendix C.

The OPE results of $T_{1_{1}}^{V}\left(q^{2}\right), T_{P_{1}}^{V, \lambda}\left(q^{2}\right)$ and $T_{P_{1}}^{V, \alpha}\left(q^{2}\right)$ are

$$
\begin{aligned}
T_{P_{1}}^{V, \mathrm{OPE}}\left(q^{2}\right)= & \left(m_{1}+m_{2}\right) \frac{\alpha_{s}}{1152 \pi^{3}} \ln \frac{-q^{2}}{\mu^{2}}\left[13-2 \ln \frac{-q^{2}}{\mu^{2}}\right] \\
& +\frac{\alpha_{s}}{18 \pi} \frac{\left\langle\bar{q}_{1} q_{1}\right\rangle+\left\langle\bar{q}_{2} q_{2}\right\rangle}{q^{2}}+\frac{5}{108} \frac{\alpha_{s}}{\pi} \frac{\left\langle\bar{q}_{1} g_{s} \sigma G q_{1}\right\rangle+\left\langle\bar{q}_{2} g_{s} \sigma G q_{2}\right\rangle}{q^{4}} \\
& +\left(0+\mathcal{O}\left(\alpha_{s}\right)\right)\left\langle\alpha_{s} G^{2}\right\rangle\left(\left\langle\bar{q}_{1} q_{1}\right\rangle+\left\langle\bar{q}_{2} q_{2}\right\rangle\right), \\
T_{1}^{V, \lambda, \mathrm{OPE}}\left(q^{2}\right)= & \left(m_{1}-m_{2}\right) \frac{\alpha_{s}}{288 \pi^{3}} \ln \frac{-q^{2}}{\mu^{2}}\left[-11+2 \ln \frac{-q^{2}}{\mu^{2}}\right] \\
& +\frac{\alpha_{s}}{9 \pi} \frac{\left\langle\bar{q}_{1} q_{1}\right\rangle-\left\langle\bar{q}_{2} q_{2}\right\rangle}{q^{2}}+\frac{173}{3456} \frac{\alpha_{s}}{\pi} \frac{\left\langle\bar{q}_{1} g_{s} \sigma G q_{1}\right\rangle-\left\langle\bar{q}_{2} g_{s} \sigma G q_{2}\right\rangle}{q^{4}} \\
& +\left(0+\mathcal{O}\left(\alpha_{s}\right)\right)\left\langle\alpha_{s} G^{2}\right\rangle\left(\left\langle\bar{q}_{1} q_{1}\right\rangle-\left\langle\bar{q}_{2} q_{2}\right\rangle\right),
\end{aligned}
$$

and

$$
T_{1 P_{1}}^{V, \sigma, \mathrm{OPE}}\left(q^{2}\right)=\left(m_{1}-m_{2}\right) \frac{\alpha_{s}}{3200 \pi^{3}} \ln \frac{-q^{2}}{\mu^{2}}\left[-57+10 \ln \frac{-q^{2}}{\mu^{2}}\right]
$$




$$
\begin{aligned}
& +\frac{\alpha_{s}}{18 \pi} \frac{\left\langle\bar{q}_{1} q_{1}\right\rangle-\left\langle\bar{q}_{2} q_{2}\right\rangle}{q^{2}}+\frac{107}{1728} \frac{\alpha_{s}}{\pi} \frac{\left\langle\bar{q}_{1} g_{s} \sigma G q_{1}\right\rangle-\left\langle\bar{q}_{2} g_{s} \sigma G q_{2}\right\rangle}{q^{4}} \\
& +\left(0+\mathcal{O}\left(\alpha_{s}\right)\right)\left\langle\alpha_{s} G^{2}\right\rangle\left(\left\langle\bar{q}_{1} q_{1}\right\rangle-\left\langle\bar{q}_{2} q_{2}\right\rangle\right),
\end{aligned}
$$

respectively. Note that $T_{1}^{V}, T_{1}^{V, \lambda}$, and $T_{1}^{V, \sigma}$ can receive contributions from ${ }^{3} P_{1}$ states because ${ }^{3} P_{1}$ states have small pseudo-tensor coupling constants due to the unequal quark masses. Consequently, we obtain the QCD sum rules

$$
\begin{aligned}
& e^{-m_{1_{P_{1}}}^{2} / M^{2}} f_{{ }_{1} P_{1}}^{\perp} f_{3,{ }^{1} P_{1}}^{V}+e^{-m_{3_{P_{1}}}^{2} / M^{2}} f_{{ }^{3} P_{1}}^{\perp} a_{0}^{\perp, 1^{3} P_{1}} f_{3,{ }^{3} P_{1}}^{V} \sigma_{3}^{V} P_{1} \\
& =\left(m_{1}+m_{2}\right) \frac{\alpha_{s}}{1152 \pi^{3}} \int_{0}^{s_{0}{ }^{s_{1}}}\left[-13+4 \ln \frac{s}{\mu^{2}}\right] e^{-s / M^{2}} d s \\
& -\frac{\alpha_{s}}{18 \pi}\left(\left\langle\bar{q}_{1} q_{1}\right\rangle+\left\langle\bar{q}_{2} q_{2}\right\rangle\right)+\frac{5}{108} \frac{\alpha_{s}}{\pi} \frac{\left\langle\bar{q}_{1} g_{s} \sigma G q_{1}\right\rangle+\left\langle\bar{q}_{2} g_{s} \sigma G q_{2}\right\rangle}{M^{2}}, \\
& e^{-m_{1_{P_{1}}}^{2} / M^{2}} f_{{ }^{1} P_{1}}^{\perp} f_{3,{ }^{1} P_{1}}^{V} \lambda_{{ }^{1} P_{1}}^{V}+e^{-m_{{ }^{2} P_{1}}^{2} / M^{2}} f_{{ }^{3} P_{1}}^{\perp} a_{0}^{\perp, 1^{3} P_{1}} f_{3,{ }^{3} P_{1}}^{V} \\
& =\left(m_{1}-m_{2}\right) \frac{\alpha_{s}}{288 \pi^{3}} \int_{0}^{s_{0}{ }^{s_{0}} P_{1}}\left[11-4 \ln \frac{s}{\mu^{2}}\right] e^{-s / M^{2}} d s \\
& -\frac{\alpha_{s}}{9 \pi}\left(\left\langle\bar{q}_{1} q_{1}\right\rangle-\left\langle\bar{q}_{2} q_{2}\right\rangle\right)+\frac{173}{3456} \frac{\alpha_{s}}{\pi} \frac{\left\langle\bar{q}_{1} g_{s} \sigma G q_{1}\right\rangle-\left\langle\bar{q}_{2} g_{s} \sigma G q_{2}\right\rangle}{M^{2}},
\end{aligned}
$$

and

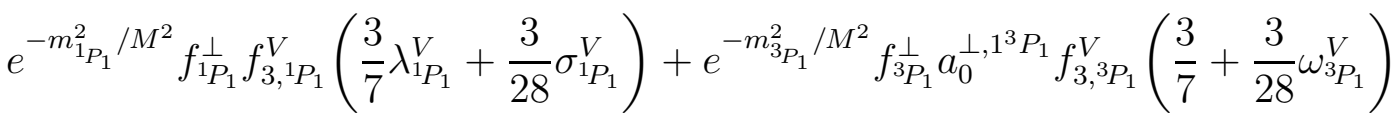

$$
\begin{aligned}
& =\left(m_{1}-m_{2}\right) \frac{\alpha_{s}}{3200 \pi^{3}} \int_{0}^{s_{0}{ }^{P_{1}}}\left[57-20 \ln \frac{s}{\mu^{2}}\right] e^{-s / M^{2}} d s \\
& -\frac{\alpha_{s}}{18 \pi}\left(\left\langle\bar{q}_{1} q_{1}\right\rangle-\left\langle\bar{q}_{2} q_{2}\right\rangle\right)+\frac{107}{1728} \frac{\alpha_{s}}{\pi} \frac{\left\langle\bar{q}_{1} g_{s} \sigma G q_{1}\right\rangle-\left\langle\bar{q}_{2} g_{s} \sigma G q_{2}\right\rangle}{M^{2}} .
\end{aligned}
$$

In Eqs. (214), the contribution originating from G-parity breaking parameters $a_{0}^{\perp, 1^{3} P_{1}} \sigma_{3}^{V} P_{1}$ relevant to LCDAs of the $1^{3} P_{1}$ state are relatively suppressed by $\mathcal{O}\left(m_{q}^{2}\right)$ and can thus be neglected. In Eqs. (215) and (216), the contributions arising from the $1^{3} P_{1}$ state are of the same order of magnitude as the G-parity breaking parameters related to the ${ }^{1} P_{1}$ state, and should be taken into account in the numerical analysis. Analogously, one needs to take into account the corrections due to the $1^{3} P_{1}$ state in Eqs. (232), (243) and (244) in the following subsections, whereas such corrections can be negligible in Eqs. (230), (231) and (242).

\subsection{2 $f_{3,{ }^{1} P_{1}}^{A}, \omega_{1 P_{1}}^{A}$ and $\sigma_{1 P_{1}}^{A}$}

The parameters $f_{3,{ }^{1} P_{1}}^{A}, \omega_{1}^{A}$ and $\sigma_{1 P_{1}}^{A}$ are defined through the matrix elements:

$$
\left\langle 0\left|J_{1, \mu}^{3, A}(0)\right| 1^{1} P_{1}(P, \lambda)\right\rangle=-f_{3,{ }^{1} P_{1}}^{A}(P \bar{z})^{2} \epsilon_{\perp, \mu}^{(\lambda)}+\mathcal{O}\left(\bar{z}_{\mu}\right),
$$




$$
\begin{aligned}
& \left\langle 0\left|J_{2, \mu}^{3, A}(0)\right| 1^{1} P_{1}(P, \lambda)\right\rangle=-f_{3,{ }^{1} P_{1}}^{A}\left\langle\alpha_{g}^{A}\right\rangle(P \bar{z})^{3} \epsilon_{\perp, \mu}^{(\lambda)}+\mathcal{O}\left(\bar{z}_{\mu}\right), \\
& \left\langle 0\left|J_{3, \mu}^{3, A}(0)\right| 1^{1} P_{1}(P, \lambda)\right\rangle=-f_{3,{ }^{1} P_{1}}^{A} \sigma_{1}^{A} P_{1}(P \bar{z})^{3} \epsilon_{\perp, \mu}^{(\lambda)}+\mathcal{O}\left(\bar{z}_{\mu}\right),
\end{aligned}
$$

where the interpolating currents have been given by Eqs. (169), (170), and (171). $\mathcal{O}\left(\bar{z}_{\mu}\right)$ contains the twist- 3 and twist- 4 corrections, and the average gluon momentum fraction $\left\langle\alpha_{g}^{A}\right\rangle$ satisfies

$$
\left\langle\alpha_{g}^{A}\right\rangle=\frac{3}{7}+\frac{3}{28} \omega_{P_{1}}^{A} .
$$

$f_{3,{ }^{1} P_{1}}^{A}, \omega_{P_{1}}^{A}$ and $\sigma_{1 P_{1}}^{A}$ can be therefore evaluated by considering the correlation functions,

$$
\begin{aligned}
& i \int d^{4} x e^{i q x}\left\langle 0\left|T\left\{J_{1, \mu}^{3, A}(x), \bar{q}_{1}(0) \bar{z}^{\lambda} \sigma_{\nu \lambda} \gamma_{5} q_{2}(0)\right\}\right| 0\right\rangle=T_{1 P_{1}}^{A}\left(q^{2}\right)(q \bar{z})^{3} g_{\mu \nu}^{\perp}+\cdots, \\
& i \int d^{4} x e^{i q x}\left\langle 0\left|T\left\{J_{2, \mu}^{3, A}(x), \bar{q}_{1}(0) \bar{z}^{\lambda} \sigma_{\nu \lambda} \gamma_{5} q_{2}(0)\right\}\right| 0\right\rangle=T_{1}^{A, \alpha_{1}}\left(q^{2}\right)(q \bar{z})^{4} g_{\mu \nu}^{\perp}+\cdots, \\
& i \int d^{4} x e^{i q x}\left\langle 0\left|T\left\{J_{3, \mu}^{3, A}(x), \bar{q}_{1}(0) \bar{z}^{\lambda} \sigma_{\nu \lambda} \gamma_{5} q_{2}(0)\right\}\right| 0\right\rangle=T_{1}^{A, \sigma}\left(q^{2}\right)(q \bar{z})^{4} g_{\mu \nu}^{\perp}+\cdots,
\end{aligned}
$$

respectively. To consider the light quark masses consistently to the $\mathcal{O}\left(\alpha_{s}\right)$, the currents needs to be replaced by the renormalized ones as

$$
\begin{aligned}
J_{1, \mu}^{3, A} \longrightarrow \bar{J}_{1, \mu}^{3, A} & =J_{1, \mu}^{3, A}+\frac{\alpha_{s}}{4 \pi} \frac{2}{9 \hat{\varepsilon}}\left(m_{1}+m_{2}\right) \bar{z}^{\alpha}(i D \cdot \bar{z})\left(\bar{q}_{2} \sigma_{\alpha \mu} \gamma_{5} q_{1}\right), \\
J_{2, \mu}^{3, A} \longrightarrow \bar{J}_{2, \mu}^{3, A} & =J_{2, \mu}^{3, A}+\frac{\alpha_{s}}{4 \pi} \frac{1}{10 \hat{\varepsilon}}\left(m_{1}+m_{2}\right) \bar{z}^{\alpha}(i D \cdot \bar{z})^{2}\left(\bar{q}_{2} \sigma_{\alpha \mu} \gamma_{5} q_{1}\right), \\
J_{3, \mu}^{3, A} \longrightarrow \bar{J}_{3, \mu}^{3, A} & =J_{3, \mu}^{3, A}-\frac{\alpha_{s}}{4 \pi} \frac{4}{45 \hat{\varepsilon}}\left(m_{1}-m_{2}\right) \bar{z}^{\alpha}(i D \cdot \bar{z})^{2}\left(\bar{q}_{2} \sigma_{\alpha \mu} \gamma_{5} q_{1}\right) .
\end{aligned}
$$

From the above results we obtain that $f_{3,{ }_{1} P_{1}}^{A}$ and $f_{3,{ }_{1} P_{1}}^{A} \omega_{{ }_{1} P_{1}}^{\perp}$ mix with $f_{{ }^{1} P_{1}}^{\perp}\left(m_{q_{1}}+m_{q_{2}}\right)$, while $f_{3,{ }^{1} P_{1}}^{A} \sigma_{{ }_{1} P_{1}}^{\perp}$ mixes with $f_{{ }^{1} P_{1}}^{\perp}\left(m_{q_{1}}-m_{q_{2}}\right)$. As our results in the previous subsection, we did not find any literature that can be used to compare with the present calculations. Again, in analogy to the discussions after Eqs. (189) and (210), the relevant parameters can mix in addition with $f_{{ }^{\perp} P_{1}}^{\perp}\left(m_{q_{1}}+m_{q_{2}}\right) a_{1}^{\perp,{ }^{1} P_{1}}$ for $f_{3,{ }^{1} P_{1}}^{A}$, and with $f_{1^{\prime} P_{1}}^{\perp}\left(m_{q_{1}} \pm m_{q_{2}}\right) a_{1}^{\perp,}{ }^{1} P_{1}$ and $f_{{ }_{1} P_{1}}^{\perp}\left(m_{q_{1}} \pm\right.$ $\left.m_{q_{2}}\right) a_{2}^{\perp,{ }^{1} P_{1}}$ for $f_{3,{ }^{1} P_{1}}^{A} \omega_{{ }_{1} P_{1}}^{\perp}$ corresponding to the upper sign and $f_{3,{ }^{1} P_{1}}^{A} \sigma_{{ }^{1} P_{1}}^{\perp}$ to the lower sign. We neglect the RG-corrections due to $\left(m_{q_{1}} \pm m_{q_{2}}\right) a_{1}^{\perp,}{ }^{1} P_{1}$ and $\left(m_{q_{1}} \pm m_{q_{2}}\right) a_{2}^{\perp,},{ }^{1} P_{1}$ in the present calculations. The RG evolutions, containing the quark mass corrections, for the relevant parameters are summarized in Appendix C.

We get

$$
\begin{aligned}
T_{1 P_{1}}^{A, \mathrm{OPE}}\left(q^{2}\right)= & -\left(m_{1}+m_{2}\right) \frac{\alpha_{s}}{1152 \pi^{3}} \ln \frac{-q^{2}}{\mu^{2}}\left[7+8 \ln \frac{-q^{2}}{\mu^{2}}\right] \\
& -\frac{\alpha_{s}}{9 \pi} \frac{\left\langle\bar{q}_{1} q_{1}\right\rangle+\left\langle\bar{q}_{2} q_{2}\right\rangle}{q^{2}}+\frac{59}{1728} \frac{\alpha_{s}}{\pi} \frac{\left\langle\bar{q}_{1} g_{s} \sigma G q_{1}\right\rangle+\left\langle\bar{q}_{2} g_{s} \sigma G q_{2}\right\rangle}{q^{4}} \\
& +\left(0+\mathcal{O}\left(\alpha_{s}\right)\right)\left\langle\alpha_{s} G^{2}\right\rangle\left(\left\langle\bar{q}_{1} q_{1}\right\rangle+\left\langle\bar{q}_{2} q_{2}\right\rangle\right),
\end{aligned}
$$




$$
\begin{aligned}
T_{1}^{A, \alpha_{1}, \mathrm{OPE}}\left(q^{2}\right)= & \left(m_{1}+m_{2}\right) \frac{\alpha_{s}}{3200 \pi^{3}} \ln \frac{-q^{2}}{\mu^{2}}\left[57-10 \ln \frac{-q^{2}}{\mu^{2}}\right] \\
& -\frac{\alpha_{s}}{18 \pi} \frac{\left\langle\bar{q}_{1} q_{1}\right\rangle+\left\langle\bar{q}_{2} q_{2}\right\rangle}{q^{2}}+\frac{29}{864} \frac{\alpha_{s}}{\pi} \frac{\left\langle\bar{q}_{1} g_{s} \sigma G q_{1}\right\rangle+\left\langle\bar{q}_{2} g_{s} \sigma G q_{2}\right\rangle}{q^{4}} \\
& +\left(0+\mathcal{O}\left(\alpha_{s}\right)\right)\left\langle\alpha_{s} G^{2}\right\rangle\left(\left\langle\bar{q}_{1} q_{1}\right\rangle+\left\langle\bar{q}_{2} q_{2}\right\rangle\right),
\end{aligned}
$$

and

$$
\begin{aligned}
T_{1}^{A, \sigma, \mathrm{OPE}}\left(q^{2}\right)= & -\left(m_{1}-m_{2}\right) \frac{\alpha_{s}}{1440 \pi^{3}} \ln \frac{-q^{2}}{\mu^{2}}\left[\frac{185}{24}-\ln \frac{-q^{2}}{\mu^{2}}\right] \\
& -\frac{\alpha_{s}}{18 \pi} \frac{\left\langle\bar{q}_{1} q_{1}\right\rangle-\left\langle\bar{q}_{2} q_{2}\right\rangle}{q^{2}}+\frac{\alpha_{s}}{27 \pi} \frac{\left\langle\bar{q}_{1} g_{s} \sigma G q_{1}\right\rangle-\left\langle\bar{q}_{2} g_{s} \sigma G q_{2}\right\rangle}{q^{4}} \\
& +\left(0+\mathcal{O}\left(\alpha_{s}\right)\right)\left\langle\alpha_{s} G^{2}\right\rangle\left(\left\langle\bar{q}_{1} q_{1}\right\rangle-\left\langle\bar{q}_{2} q_{2}\right\rangle\right) .
\end{aligned}
$$

The resulting QCD sum rules read

$$
\begin{aligned}
& e^{-m_{1_{P_{1}}}^{2} / M^{2}} f_{{ }_{1} P_{1}}^{\perp} f_{3,{ }^{1} P_{1}}^{A}+e^{-m_{3}^{2}{ }_{P_{1}} / M^{2}} f_{{ }^{3} P_{1}}^{\perp} a_{0}^{\perp, 1^{3} P_{1}} f_{3,{ }^{3} P_{1}}^{A} \lambda_{3 P_{1}}^{A} \\
& =\left(m_{1}+m_{2}\right) \frac{\alpha_{s}}{1152 \pi^{3}} \int_{0}^{s_{0} P_{1}}\left(7+16 \ln \frac{s}{\mu^{2}}\right) e^{-s / M^{2}} d s \\
& +\frac{\alpha_{s}}{9 \pi}\left(\left\langle\bar{q}_{1} q_{1}\right\rangle+\left\langle\bar{q}_{2} q_{2}\right\rangle\right)+\frac{59}{1728} \frac{\alpha_{s}}{\pi} \frac{\left\langle\bar{q}_{1} g_{s} \sigma G q_{1}\right\rangle+\left\langle\bar{q}_{2} g_{s} \sigma G q_{2}\right\rangle}{M^{2}}, \\
& e^{-m_{1}^{2} P_{1} / M^{2}} f_{{ }_{1} P_{1}}^{\perp} f_{3,{ }^{1} P_{1}}^{A}\left(\frac{3}{7}+\frac{3}{28} \omega_{{ }^{\prime} P_{1}}^{A}\right)+e^{-m_{{ }^{2} P_{1}}^{2} / M^{2}} f_{{ }^{3} P_{1}}^{\perp} a_{0}^{\perp, 1^{3} P_{1}} f_{3,{ }^{3} P_{1}}^{A}\left(\frac{3}{7} \lambda_{{ }^{3} P_{1}}^{A}+\frac{3}{28} \sigma_{{ }^{3} P_{1}}^{A}\right) \\
& =\left(m_{1}+m_{2}\right) \frac{\alpha_{s}}{3200 \pi^{3}} \int_{0}^{s_{0} P_{1}}\left(-57+20 \ln \frac{s}{\mu^{2}}\right) e^{-s / M^{2}} d s \\
& +\frac{\alpha_{s}}{18 \pi}\left(\left\langle\bar{q}_{1} q_{1}\right\rangle+\left\langle\bar{q}_{2} q_{2}\right\rangle\right)+\frac{29}{864} \frac{\alpha_{s}}{\pi} \frac{\left\langle\bar{q}_{1} g_{s} \sigma G q_{1}\right\rangle+\left\langle\bar{q}_{2} g_{s} \sigma G q_{2}\right\rangle}{M^{2}},
\end{aligned}
$$

and

$$
\begin{aligned}
& e^{-m_{1}^{2}{ }_{P_{1}} / M^{2}} f_{{ }_{1} P_{1}}^{\perp} f_{3,{ }^{1} P_{1}}^{A} \sigma_{1 P_{1}}^{A}+e^{-m_{3}^{2}{ }_{P_{1}} / M^{2}} f_{{ }_{3} P_{1}}^{\perp} a_{0}^{\perp, 1^{3} P_{1}} f_{3,{ }^{3} P_{1}}^{A} \\
& =\left(m_{1}-m_{2}\right) \frac{\alpha_{s}}{1440 \pi^{3}} \int_{0}^{s_{0} P_{1}}\left(\frac{185}{24}-2 \ln \frac{s}{\mu^{2}}\right) e^{-s / M^{2}} d s \\
& +\frac{\alpha_{s}}{18 \pi}\left(\left\langle\bar{q}_{1} q_{1}\right\rangle-\left\langle\bar{q}_{2} q_{2}\right\rangle\right)+\frac{\alpha_{s}}{27 \pi} \frac{\left\langle\bar{q}_{1} g_{s} \sigma G q_{1}\right\rangle-\left\langle\bar{q}_{2} g_{s} \sigma G q_{2}\right\rangle}{M^{2}} .
\end{aligned}
$$




\subsection{3 $f_{3,{ }^{1} P_{1}}^{\perp}, \lambda^{\perp}{ }_{P_{1}}$, and $\sigma_{{ }^{1} P_{1}}^{\perp}$}

The coupling constants $f_{3,{ }_{1} P_{1}}^{\perp}, \lambda^{1} P_{1}$, and $\sigma^{1} P_{1}$ for $1^{1} P_{1}$ mesons are defined as the following matrix elements

$$
\begin{aligned}
& \left\langle 0\left|J_{3}^{3, \perp}(0)\right| 1^{1} P_{1}(P, \lambda)\right\rangle=i f_{3,{ }^{1} P_{1}}^{\perp}(P \bar{z})^{2}\left(\epsilon^{(\lambda)} \bar{z}\right), \\
& \left\langle 0\left|J_{1}^{3, \perp}(0)\right| 1^{1} P_{1}(P, \lambda)\right\rangle=i f_{3,{ }_{1} P_{1}}^{\perp} \lambda^{\perp} P_{1}(P \bar{z})\left(\epsilon^{(\lambda)} \bar{z}\right),
\end{aligned}
$$

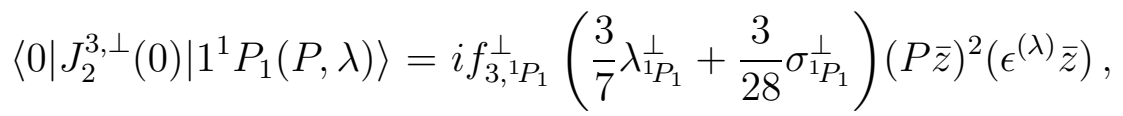

where the interpolating currents have been given by Eqs. (184), (185), and (186). To calculate these three parameters, we consider the following correlation functions,

$$
\begin{aligned}
& \int d^{4} x e^{i q x}\left\langle 0\left|T\left\{J_{3}^{3, \perp}(x), \bar{q}_{1}(0) \bar{z}^{\nu} \sigma_{\mu \nu} \gamma_{5} q_{2}(0)\right\}\right| 0\right\rangle=-T_{P_{P_{1}}}^{\perp}\left(q^{2}\right)(q \bar{z})^{3} \bar{z}_{\mu}+\cdots, \\
& \int d^{4} x e^{i q x}\left\langle 0\left|T\left\{J_{1}^{3 \perp}(x), \bar{q}_{1}(0) \bar{z}^{\nu} \sigma_{\mu \nu} \gamma_{5} q_{2}(0)\right\}\right| 0\right\rangle=-T_{{ }_{1} P_{1}}^{\perp, \lambda}\left(q^{2}\right)(q \bar{z})^{2} \bar{z}_{\mu}+\cdots \\
& \int d^{4} x e^{i q x}\left\langle 0\left|T\left\{J_{2}^{3, \perp}(x), \bar{q}_{1}(0) \bar{z}^{\nu} \sigma_{\mu \nu} \gamma_{5} q_{2}(0)\right\}\right| 0\right\rangle=-T_{P_{1}}^{\perp, \sigma}\left(q^{2}\right)(q \bar{z})^{3} \bar{z}_{\mu}+\cdots
\end{aligned}
$$

It is interesting to note that the above correlation functions receive no contributions from $1^{-}$states. The OPE results of $T_{1_{1}}^{\perp}\left(q^{2}\right)$ have the following forms

$$
\begin{aligned}
& T_{P_{1}}^{\perp, \mathrm{OPE}}\left(q^{2}\right) \\
& \left.=-\frac{\alpha_{s}}{720 \pi^{3}} q^{2} \ln \frac{-q^{2}}{\mu^{2}}-\frac{\left\langle\alpha_{s} G^{2}\right\rangle}{36 \pi q^{2}}+\frac{16 \pi \alpha_{s}}{27 q^{4}}\left(\left\langle\bar{q}_{1} q_{1}\right\rangle^{2}+\left\langle\bar{q}_{2} q_{2}\right\rangle^{2}\right)+\frac{8 \pi \alpha_{s}}{9 q^{4}}\left\langle\bar{q}_{1} q_{1}\right\rangle\left\langle\bar{q}_{2} q_{2}\right\rangle\right) \\
& \quad+\frac{\alpha_{s}}{18 \pi q^{2}}\left[\frac{9}{2}\left(m_{1}\left\langle\bar{q}_{1} q_{1}\right\rangle+m_{2}\left\langle\bar{q}_{2} q_{2}\right\rangle\right)-\left(m_{1}\left\langle\bar{q}_{2} q_{2}\right\rangle+m_{2}\left\langle\bar{q}_{1} q_{1}\right\rangle\right)\left(\ln \frac{-q^{2}}{\mu^{2}}+\frac{1}{3}\right)\right], \quad \\
& T_{1 P_{1}}^{\perp, \lambda, \mathrm{OPE}}\left(q^{2}\right)=0 \cdot\left\langle\alpha_{s} G^{2}\right\rangle-\frac{16 \pi \alpha_{s}}{27 q^{4}}\left\langle\left\langle\bar{q}_{1} q_{1}\right\rangle^{2}-\left\langle\bar{q}_{2} q_{2}\right\rangle^{2}\right) \\
& \quad-\frac{\alpha_{s}}{18 \pi q^{2}}\left[7\left(m_{1}\left\langle\bar{q}_{1} q_{1}\right\rangle-m_{2}\left\langle\bar{q}_{2} q_{2}\right\rangle\right)+4\left(m_{1}\left\langle\bar{q}_{2} q_{2}\right\rangle-m_{2}\left\langle\bar{q}_{1} q_{1}\right\rangle\right)\left(\ln \frac{-q^{2}}{\mu^{2}}-\frac{2}{3}\right)\right] . \\
& T_{1 P_{1}}^{\perp, \sigma \mathrm{OPE}}\left(q^{2}\right) \\
& =-\frac{\alpha_{s}}{36 \pi q^{2}}\left[5\left(m_{1}\left\langle\bar{q}_{1} q_{1}\right\rangle-m_{2}\left\langle\bar{q}_{2} q_{2}\right\rangle\right)+4\left(m_{1}\left\langle\bar{q}_{2} q_{2}\right\rangle-m_{2}\left\langle\bar{q}_{1} q_{1}\right\rangle\right)\left(\ln \frac{-q^{2}}{\mu^{2}}-\frac{7}{12}\right)\right] \\
& \quad+0 \cdot\left\langle\alpha_{s} G^{2}\right\rangle+0 \cdot\left\langle\bar{q}_{1} q_{1}\right\rangle\left\langle\bar{q}_{2} q_{2}\right\rangle+\left(0+\mathcal{O}\left(\alpha_{s}^{2}\right)\right)\left(\left\langle\bar{q}_{1} q_{1}\right\rangle^{2},\left\langle\bar{q}_{2} q_{2}\right\rangle^{2}\right) .
\end{aligned}
$$


Consequently, we obtain the sum rules

$$
\begin{aligned}
& e^{-m_{1}^{2}{ }_{P_{1}} / M^{2}} m_{1} P_{1} f_{1 P_{1}}^{\perp} f_{3,{ }^{1} P_{1}}^{\perp}+e^{-m_{3 P_{1}}^{2} / M^{2}} m_{3} P_{1} f_{3 P_{1}}^{\perp} a_{0}^{\perp, 1^{3} P_{1}} f_{3,{ }^{3} P_{1}}^{\perp} \sigma_{3 P_{1}}^{\perp} \\
& =\frac{\alpha_{s}}{720 \pi^{3}} \int_{0}^{{ }^{s_{0} P_{1}}} d s s e^{-s / M^{2}}+\frac{\left\langle\alpha_{s} G^{2}\right\rangle}{36 \pi}+\frac{16 \pi \alpha_{s}}{27 M^{2}}\left(\left\langle\bar{q}_{1} q_{1}\right\rangle^{2}+\left\langle\bar{q}_{2} q_{2}\right\rangle^{2}\right)+\frac{8 \pi \alpha_{s}}{9 M^{2}}\left\langle\bar{q}_{1} q_{1}\right\rangle\left\langle\bar{q}_{2} q_{2}\right\rangle \\
& +\frac{\alpha_{s}}{18 \pi}\left[-\frac{9}{2}\left(m_{1}\left\langle\bar{q}_{1} q_{1}\right\rangle+m_{2}\left\langle\bar{q}_{2} q_{2}\right\rangle\right)\right. \\
& \left.+\left(m_{1}\left\langle\bar{q}_{2} q_{2}\right\rangle+m_{2}\left\langle\bar{q}_{1} q_{1}\right\rangle\right)\left(\frac{1}{3}-\gamma_{E}-\ln \frac{\mu^{2}}{M^{2}}+\operatorname{Ei}\left(-\frac{s_{0}^{1} P_{1}}{M^{2}}\right)\right)\right] \\
& e^{-m_{1_{P_{1}}}^{2} / M^{2}} m_{1} P_{1} f_{{ }^{1} P_{1}}^{\perp} f_{3,{ }^{1} P_{1}}^{\perp} \lambda_{{ }_{1} P_{1}}^{\perp}+e^{-m_{3_{P_{1}}}^{2} / M^{2}} m_{3} P_{1} f_{3 P_{1}}^{\perp} a_{0}^{\perp, 1^{3} P_{1}} f_{3,{ }^{3} P_{1}}^{\perp} \\
& =-\frac{16 \pi \alpha_{s}}{27 M^{2}}\left(\left\langle\bar{q}_{1} q_{1}\right\rangle^{2}-\left\langle\bar{q}_{2} q_{2}\right\rangle^{2}\right)+\frac{\alpha_{s}}{18 \pi}\left[7\left(m_{1}\left\langle\bar{q}_{1} q_{1}\right\rangle-m_{2}\left\langle\bar{q}_{2} q_{2}\right\rangle\right)\right. \\
& \left.+4\left(m_{1}\left\langle\bar{q}_{2} q_{2}\right\rangle-m_{2}\left\langle\bar{q}_{1} q_{1}\right\rangle\right)\left(-\frac{2}{3}-\gamma_{E}-\ln \frac{\mu^{2}}{M^{2}}+\operatorname{Ei}\left(-\frac{s_{0}^{1} P_{1}}{M^{2}}\right)\right)\right],
\end{aligned}
$$

and

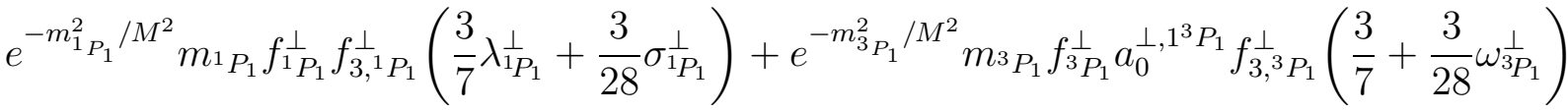

$$
\begin{aligned}
& =\frac{\alpha_{s}}{36 \pi}\left[5\left(m_{1}\left\langle\bar{q}_{1} q_{1}\right\rangle-m_{2}\left\langle\bar{q}_{2} q_{2}\right\rangle\right)\right. \\
& \left.+4\left(m_{1}\left\langle\bar{q}_{2} q_{2}\right\rangle-m_{2}\left\langle\bar{q}_{1} q_{1}\right\rangle\right)\left(-\frac{7}{12}-\gamma_{E}-\ln \frac{\mu^{2}}{M^{2}}+\operatorname{Ei}\left(-\frac{s_{0}^{1} P_{1}}{M^{2}}\right)\right)\right] .
\end{aligned}
$$

Note that the calculation of Eq. (236) is actually analogous to that of $f_{3 \rho}^{T}$ for the $\rho$ [9], where there is no $\gamma_{5}$ for the $\rho$. Neglecting the mass corrections in Eq. (242), our perturbative and gluon-condensate contributions agree with Eq. (C.15) in Ref. [9], but the term of dimension-6 is different from theirs, where the sign difference due to $\gamma_{5}$ has been considered.

\subsection{Results}

In the numerical analysis, we use $f_{3} P_{1}, f_{1}^{\perp}, s_{0}^{3} P_{1}, s_{0}^{1} P_{1}$, and the masses for axial-vector mesons, which have been obtained in the previous section, as inputs. We also adopt the parameters given in Appendix C. The Borel window can thus be determined by means of that the contributions arising from the higher resonances, including the continuum, and from the term of the highest dimension in the OPE series are well under control. We find that the suitable Borel window is $1.5 \mathrm{GeV}^{2}<M^{2}<2.5 \mathrm{GeV}^{2}$ except that the Borel window is $2.5 \mathrm{GeV}^{2}<M^{2}<3.5 \mathrm{GeV}^{2}$ for the $\omega_{3 P_{1}}^{V}$ sum rule. The reason that we have to choose a 
higher Borel window for the $\omega_{3 P_{1}}^{V}$ sum rule is because a lower Borel mass will lead to a slowly convergence at the quark-gluon level; for instance, at $M^{2}=2.0 \mathrm{GeV}^{2}$, the highest dimension (dimension $=6$ ) term still gives a large correction, $\sim 33 \%$, at the quark-gluon level. Instead, during $2.5 \mathrm{GeV}^{2}<M^{2}<3.5 \mathrm{GeV}^{2}$, the contribution arising from the highest dimension term is about $14 \% \sim 5 \%$. On the other hand, because the average gluon momentum fraction in an axial-vector meson is not less than zero, therefore we should have $\omega_{3}^{V} \geq-4$, so that the region for $M^{2}<1.6 \mathrm{GeV}^{2}$ is strongly disfavored by the $\omega_{3}^{V} P_{1}$ sum rule.

As we evaluate G-parity invariant parameters for $1^{3} P_{1}$ states, the corrections receiving from $1^{1} P_{1}$ states are negligible, and vice versa. Nevertheless, if calculating G-parity violating parameters for the $K_{1 A}\left(K_{1 B}\right)$ state, the corrections originating from the $K_{1 B}\left(K_{1 A}\right)$ state cannot be ignored. In the numerical study we use $a_{0}^{\perp, K_{1 A}}$ and $a_{0}^{\|, K_{1 B}}$, given in Table 4 , as inputs. To obtain the relevant parameter in a sum rule, we replace the other twist-3 parameters using the corresponding sum rules. For instance, in evaluating $\sigma_{3}^{V}$, we substitute $f_{3,{ }^{3} P_{1}}^{V}$ and $f_{3,{ }^{1} P_{1}}^{V}$ by the expressions given in Eqs. (163) and (214) into Eq. (165). Note that for $\omega_{3 P_{1}}^{V}$, because the working Borel window is different from the others, we thus adopt directly the values of $f_{3,{ }^{3} P_{1}}^{V}$ given in Table 5 in the study.

We summarize the numerical results in Tables 5 and 6, where the theoretical errors are due to variation of all inputs and the predictive uncertainty within the Borel window. To illustrate the sum rule results, we plot the parameters for the $a_{1}(1260), K_{1 A}$ as functions of the Borel mass squared in Figs. 4 and $b_{1}(1235), K_{1 B}$ in Fig. 15, where the central values of input parameters given in Tables 2, 3, 4, and in Appendix C are used. For simplicity, we do not plot the results for $f_{1}, f_{8}, h_{1}$ and $h_{8}$, which are analogous to the above ones. The $f_{3,{ }^{3} P_{1}}^{\perp}$ sum rule results obtained from Eq. (199) and from Eq. (193) are depicted. Because the sum rule given in Eq. (193) exhibits badly convergent behavior (see the discussion after Eq. (195)), we therefore choose to use the diagonal sum rule given in Eq. (199), where the lowest lying pseudoscalar contribution is substituted by using Eq. (200). Although the sum rule in Eq. (199) cannot determine the sign of $f_{3,{ }^{3} P_{1}}^{\perp}$, however there are two reasons that its sign should be negative. First, Eq. (193) yields a negative $f_{3,{ }^{3} P_{1}}^{\perp}$ at the large $M^{2}$. Second, only a negative $f_{3,{ }^{3} P_{1}}^{\perp}$ can result in a physical value of $\omega_{3{ }_{1} P_{1}}^{\perp}$ that should satisfy $0 \leq \omega_{3}^{\perp} P_{1} \leq 16 / 3$.

Finally, it should be noted that the main contributions of some sum rules for parameters are due to the quark and quark-gluon condensates. These sum rules may suffer from the contributions of higher resonances at hadronic level and radiative corrections at quark-gluon level. The two effects may partially cancel each other and give about $\lesssim 10 \%$ corrections to the numerical results for parameters. See also the discussions in subsection 4.4.3. Here we do not include these effects in Tables 5 and 6 . 
Table 5: Parameters for twist-3 distribution amplitudes of $1^{3} P_{1}$ mesons at the scales $\mu=1$ $\mathrm{GeV}$ and $2.2 \mathrm{GeV}$ (shown in parentheses), where $f_{3,{ }^{2} P_{1}}^{V}, f_{3,{ }^{3} P_{1}}^{A}$, and $f_{3,{ }^{3} P_{1}}^{\perp}$ are in units of $\mathrm{GeV}^{2}$.

\begin{tabular}{|c|cccc|}
\hline \hline & $a_{1}(1260)$ & $f_{1}$ & $f_{8}$ & $K_{1 A}$ \\
\hline$f_{3,{ }^{V} P_{1}}^{V}$ & $0.0055 \pm 0.0027$ & $0.0055 \pm 0.0027$ & $0.0054 \pm 0.0027$ & $0.0052 \pm 0.0027$ \\
& $(0.0036 \pm 0.0018)$ & $(0.0036 \pm 0.0018)$ & $(0.0035 \pm 0.0018)$ & $(0.0034 \pm 0.0018)$ \\
\hline$\omega^{V}{ }_{P_{1}}$ & $-2.9 \pm 0.9$ & $-2.8 \pm 0.9$ & $-3.0 \pm 1.1$ & $-3.1 \pm 1.1$ \\
& $(-2.9 \pm 0.9)$ & $(-2.8 \pm 0.9)$ & $(-3.0 \pm 1.0)$ & $(-3.1 \pm 1.1)$ \\
\hline$\sigma^{V}{ }^{V} P_{1}$ & - & - & - & $-0.13 \pm 0.16$ \\
& - & - & - & $(-0.13 \pm 0.16)$ \\
\hline \hline$f_{3,{ }^{3} P_{1}}^{A}$ & $0.0022 \pm 0.0009$ & $0.0022 \pm 0.0009$ & $0.0028 \pm 0.0009$ & $0.0026 \pm 0.0013$ \\
& $(0.0012 \pm 0.0005)$ & $(0.0012 \pm 0.0005)$ & $(0.0015 \pm 0.0005)$ & $(0.0014 \pm 0.0007)$ \\
\hline$\lambda_{{ }^{A} P_{1}}^{A}$ & - & - & - & $0.57 \pm 0.39$ \\
& - & - & - & $(0.70 \pm 0.46)$ \\
\hline$\sigma_{3 P_{1}}^{A}$ & - & - & - & $2.4 \pm 2.0$ \\
& - & - & - & $(2.4 \pm 2.0)$ \\
\hline \hline$f_{3,{ }^{3} P_{1}}^{\perp}$ & $-0.013 \pm 0.002$ & $-0.012 \pm 0.002$ & $-0.012 \pm 0.002$ & $-0.012 \pm 0.002$ \\
& $(-0.009 \pm 0.002)$ & $(-0.009 \pm 0.002)$ & $(-0.009 \pm 0.002)$ & $(-0.009 \pm 0.002)$ \\
\hline$\omega^{\perp} P_{P_{1}}$ & $-3.7 \pm 0.4$ & $-3.4 \pm 0.4$ & $-3.2 \pm 0.6$ & $-3.4 \pm 0.6$ \\
& $(-2.9 \pm 0.3)$ & $(-2.6 \pm 0.3)$ & $(-2.4 \pm 0.4)$ & $(-2.6 \pm 0.4)$ \\
\hline$\sigma_{3{ }^{\perp} P_{1}}$ & - & - & - & $0.07 \pm 0.21$ \\
& - & - & - & $(0.05 \pm 0.15)$ \\
\hline \hline
\end{tabular}

\section{Models for LCDAs}

\subsection{Two-parton LCDAs of twist-two}

We have calculated the first few Gegenbauer moments of leading-twist light-cone distribution amplitudes of $1^{3} P_{1}$ and $1^{1} P_{1}$ mesons in Sec. 4.4 using the QCD sum rule technique. The Gegenbauer moments of higher conformal spins may not be predictive in the QCD sum rule approach owing to the divergence of the OPE series for relevant correlation functions. Therefore the models for light-cone distribution amplitudes depend on the truncated conformal expansions with the reliable Gegenbauer moments.

Here we take into account the approximate forms of twist- 2 distributions for $1^{3} P_{1}$ mesons as follows:

$$
\begin{aligned}
\Phi_{\|}(u) & =6 u \bar{u}\left[1+3 a_{1}^{\|} \xi+a_{2}^{\|} \frac{3}{2}\left(5 \xi^{2}-1\right)\right], \\
\Phi_{\perp}(u) & =6 u \bar{u}\left[a_{0}^{\perp}+3 a_{1}^{\perp} \xi+a_{2}^{\perp} \frac{3}{2}\left(5 \xi^{2}-1\right)\right],
\end{aligned}
$$


Table 6: The same as Table 5 except for $1^{1} P_{1}$ mesons, where $f_{3,{ }^{1} P_{1}}^{V}, f_{3,{ }^{1} P_{1}}^{A}$, and $f_{3,{ }^{1} P_{1}}^{\perp}$ are in units of $\mathrm{GeV}^{2}$.

\begin{tabular}{|c|c|c|c|c|}
\hline & $b_{1}(1235)$ & $h_{1}$ & $h_{8}$ & $K_{1 B}$ \\
\hline \multirow{2}{*}{$\overline{f_{3,{ }^{1} P_{1}}^{V}}$} & $0.0052 \pm 0.0018$ & $0.0046 \pm 0.0021$ & $0.0045 \pm 0.0020$ & $0.0049 \pm 0.0021$ \\
\hline & $(0.0030 \pm 0.0011)$ & $(0.0027 \pm 0.0012)$ & $(0.0027 \pm 0.0012)$ & $(0.0029 \pm 0.0012)$ \\
\hline \multirow[t]{2}{*}{$\lambda_{{ }^{\prime} P_{1}}^{V}$} & - & - & - & $0.07 \pm 0.19$ \\
\hline & - & - & - & $(0.09 \pm 0.24)$ \\
\hline \multirow[t]{2}{*}{$\sigma_{{ }_{P_{1}}}^{V}$} & - & - & - & $0.35 \pm 0.73$ \\
\hline & - & - & - & $(0.31 \pm 0.68)$ \\
\hline \multirow[t]{2}{*}{$f_{3,{ }^{1} P_{1}}^{A}$} & $-0.0058 \pm 0.0023$ & $-0.0053 \pm 0.0023$ & $-0.0055 \pm 0.0023$ & $-0.0065 \pm 0.0029$ \\
\hline & $(-0.0036 \pm 0.0014)$ & $(-0.0033 \pm 0.0014)$ & $(-0.0035 \pm 0.0014)$ & $(-0.0041 \pm 0.0018)$ \\
\hline \multirow{2}{*}{$\omega_{1}^{A}$} & $-1.5 \pm 0.4$ & $-1.9 \pm 0.6$ & $-3.5 \pm 1.0$ & $-1.9 \pm 0.6$ \\
\hline & $(-1.4 \pm 0.3)$ & $(-1.7 \pm 0.4)$ & $(-2.9 \pm 0.8)$ & $(-1.7 \pm 0.4)$ \\
\hline \multirow[t]{2}{*}{$\sigma_{{ }^{\prime} P_{1}}^{A}$} & - & - & - & $-0.06 \pm 0.05$ \\
\hline & - & - & - & $(-0.05 \pm 0.04)$ \\
\hline \multirow[t]{2}{*}{$f_{3,{ }^{1} P_{1}}^{\perp}$} & $0.011 \pm 0.006$ & $0.012 \pm 0.006$ & $0.012 \pm 0.005$ & $0.012 \pm 0.005$ \\
\hline & $(0.006 \pm 0.003)$ & $(0.006 \pm 0.003)$ & $(0.006 \pm 0.003)$ & $(0.006 \pm 0.003)$ \\
\hline \multirow[t]{2}{*}{$\lambda_{{ }^{\prime} P_{1}}^{\perp}$} & - & - & - & $0.17 \pm 0.17$ \\
\hline & - & - & - & $(0.25 \pm 0.25)$ \\
\hline \multirow[t]{2}{*}{$\sigma_{{ }^{\prime} P_{1}}^{\perp}$} & - & - & - & $-0.71 \pm 0.53$ \\
\hline & - & - & - & $(-0.76 \pm 0.56)$ \\
\hline
\end{tabular}

where $\xi=2 u-1$ and $a_{1}^{\|}, a_{0}^{\perp}, a_{2}^{\perp}$ are non-zero only for strange mesons. The above LCDAs of pure $1^{3} P_{1}$ states are normalized as the normalization conditions

$$
\begin{aligned}
& \int_{0}^{1} d u \Phi_{\|}(u)=1, \\
& \int_{0}^{1} d u \Phi_{\perp}(u)=a_{0}^{\perp} .
\end{aligned}
$$

In our convention, $u$ is the momentum fraction carried by the $q_{1}$ quark in an axial-vector meson (and is therefore equivalent to the momentum fraction carried by the $s$ quark in a strange meson). Note that in this paper the strange mesons that we discuss should contain an $s$ quark, while for the LCDAs of strange mesons involving an $\bar{s}$, the replacement $u \leftrightarrow 1-u$ has to be made, namely $\xi \rightarrow-\xi$. As for $1^{1} P_{1}$ mesons, we take the following approximation (see also the discussions given in Ref. [18]):

$$
\begin{aligned}
& \Phi_{\|}(u)=6 u \bar{u}\left[a_{0}^{\|}+3 a_{1}^{\|} \xi+a_{2}^{\|} \frac{3}{2}\left(5 \xi^{2}-1\right)\right], \\
& \Phi_{\perp}(u)=6 u \bar{u}\left[1+3 a_{1}^{\perp} \xi+a_{2}^{\perp} \frac{3}{2}\left(5 \xi^{2}-1\right)\right],
\end{aligned}
$$



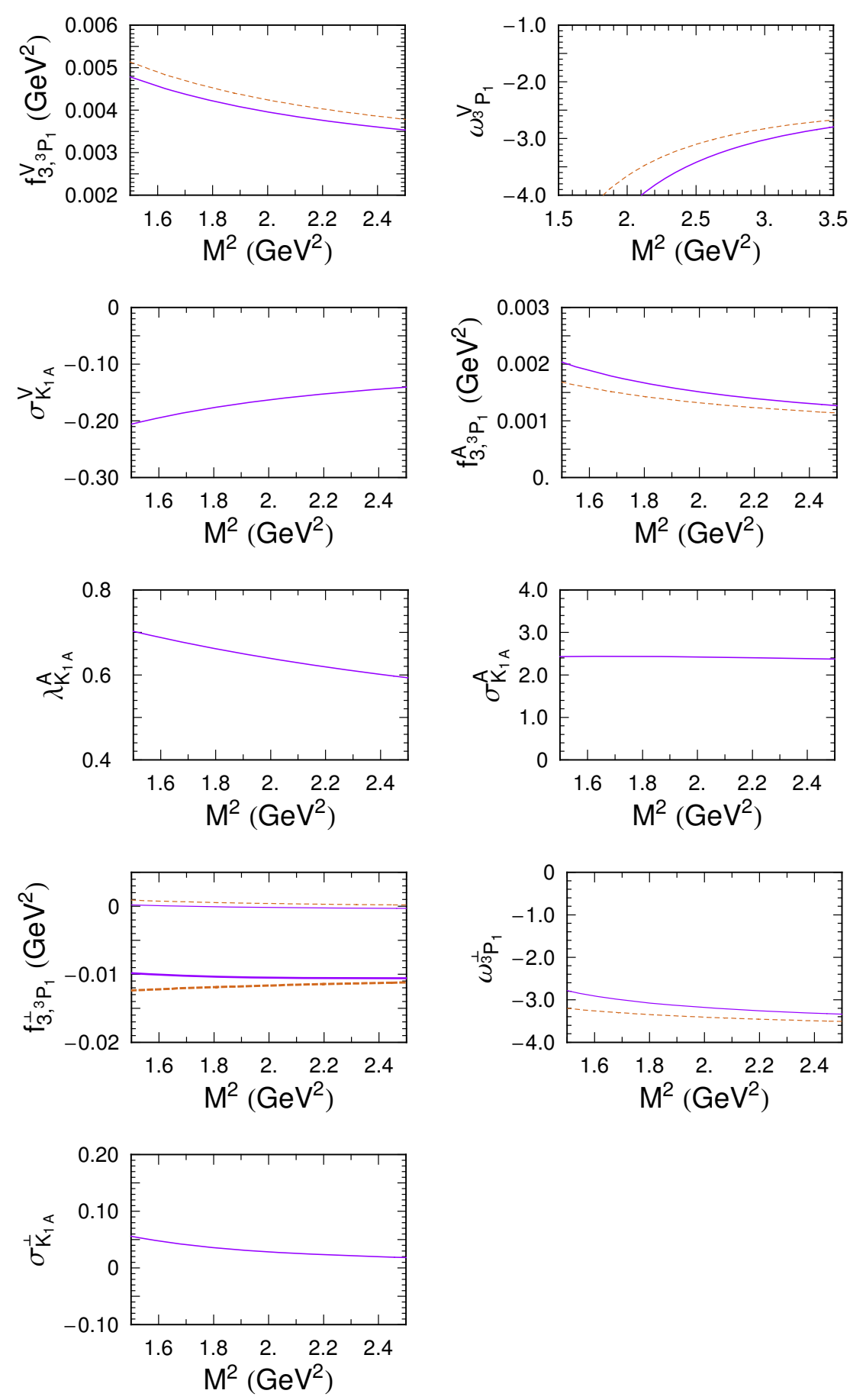

Figure 4: Some relevant parameters in determinations of three-parton distribution amplitudes of twist-3 for the $K_{1 A}$ (solid curve) and $a_{1}(1260)$ (dashed curve) as functions of the Borel mass squared, where the central values of input parameters have been used. The renormalization scale is set at $\mu \simeq 1.4 \mathrm{GeV}$ except that $\omega_{3 P_{1}}^{V}$ is at $\mu \simeq 1.7 \mathrm{GeV}$. For $f_{3,{ }_{3} P_{1}}^{\perp}$, the lower two curves are derived from Eqs. (199) and (200), and the upper two curves from Eq. (193). 

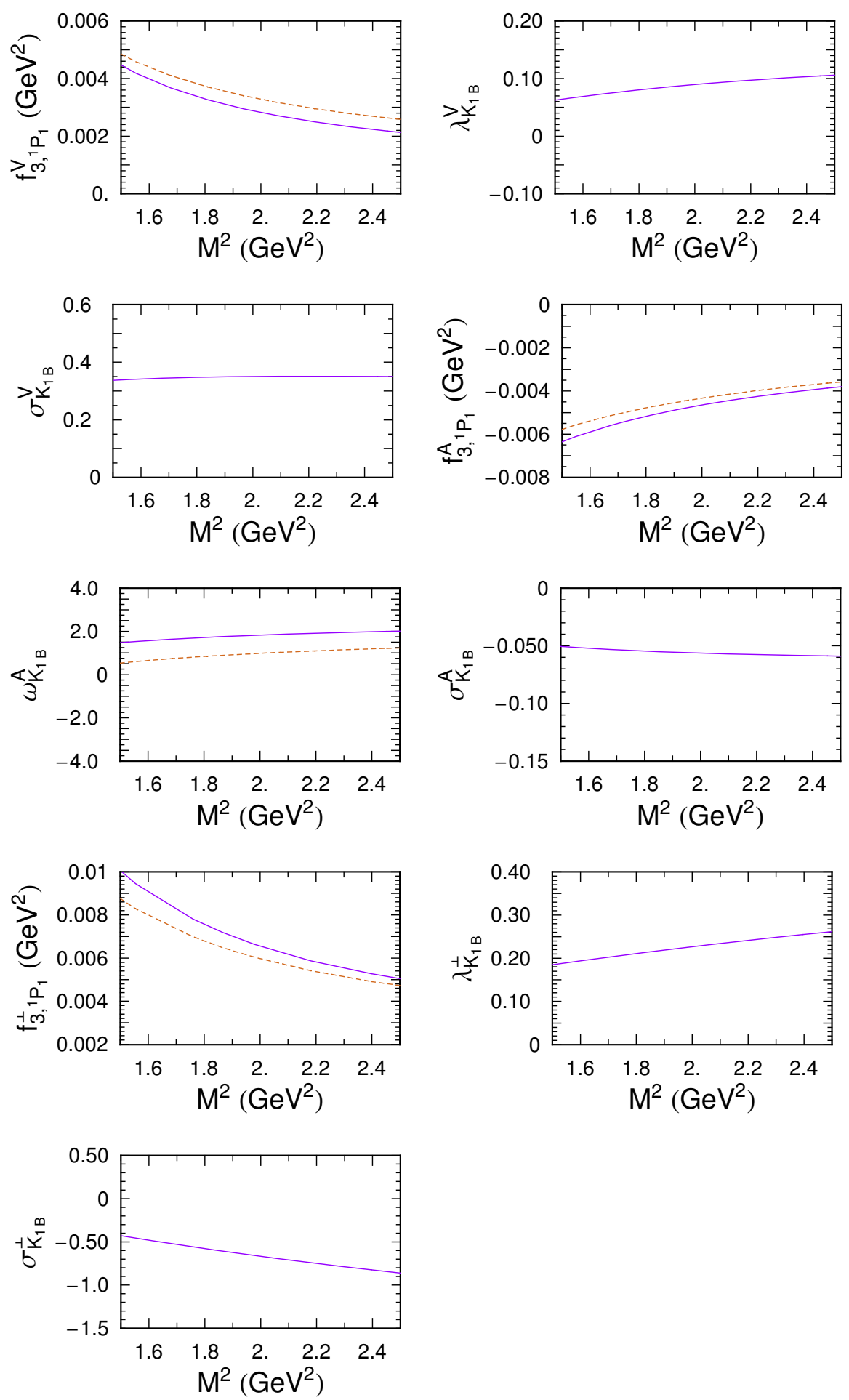

Figure 5: Some relevant parameters in determinations of three-parton distribution amplitudes of twist-3 for the $K_{1 B}$ (solid curve) and $b_{1}(1235)$ (dashed curve) as functions of the Borel mass squared, where the central values of input parameters have been used. The renormalization scale is set at $\mu \simeq 1.4 \mathrm{GeV}$. 
where $a_{0}^{\|}, a_{2}^{\|}$, and $a_{1}^{\perp}$ are non-zero only for strange mesons, so that the LCDAs of pure $1^{1} P_{1}$ states are normalized as the normalization conditions

$$
\begin{aligned}
& \int_{0}^{1} d u \Phi_{\|}(u)=a_{0}^{\|} \\
& \int_{0}^{1} d u \Phi_{\perp}(u)=1 .
\end{aligned}
$$

Due to mixtures, we define the LCDAs of physical $h_{1}(1170), h_{1}(1380), f_{1}(1285), f_{1}(1420)$ mesons in Appendix D, and, on other hand, the LCDAs for $K_{1}(1270)$ and $K_{1}(1400)$ are consequently given by

$$
\begin{gathered}
\Phi_{\|}^{K_{1}(1270)}(u)=\frac{f_{K_{1 A}} m_{K_{1 A}}}{f_{K_{1}(1270)} m_{K_{1}(1270)}} \Phi_{\|}^{K_{1 A}}(u) \sin \theta_{K}+\frac{f_{K_{1 B}} m_{K_{1 B}}}{f_{K_{1}(1270)} m_{K_{1}(1270)}} \Phi_{\|}^{K_{1 B}}(u) \cos \theta_{K}, \\
\Phi_{\|}^{K_{1}(1400)}(u)=\frac{f_{K_{1 A}} m_{K_{1 A}}}{f_{K_{1}(1400)} m_{K_{1}(1400)}} \Phi_{\|}^{K_{1 A}}(u) \cos \theta_{K}-\frac{f_{K_{1 B}} m_{K_{1 B}}}{f_{K_{1}(1400)} m_{K_{1}(1400)}} \Phi_{\|}^{K_{1 B}}(u) \sin \theta_{K}, \\
\Phi_{\perp}^{K_{1}(1270)}(u)=\frac{f_{K_{1 A}}^{\perp}}{f_{K_{1}(1270)}^{\perp}} \Phi_{\perp}^{K_{1 A}}(u) \sin \theta_{K}+\frac{f_{K_{1 B}}^{\perp}}{f_{K_{1}(1270)}^{\perp}} \Phi_{\perp}^{K_{1 B}}(u) \cos \theta_{K}, \\
\Phi_{\perp}^{K_{1}(1400)}(u)=\frac{f_{K_{1 A}}^{\perp}}{f_{K_{1}(1400)}^{\perp}} \Phi_{\perp}^{K_{1 A}}(u) \cos \theta_{K}-\frac{f_{K_{1 B}}^{\perp}}{f_{K_{1}(1400)}^{\perp}} \Phi_{\perp}^{K_{1 B}}(u) \sin \theta_{K} .
\end{gathered}
$$

In Figs. 6 and 7, we plot the twist-2 light-cone distribution amplitudes for $1^{1} P_{1}$ and $1^{3} P_{1}$ states, including results for the physical mesons, $h_{1}(1170), h_{1}(1380), f_{1}(1285), f_{1}(1420)$, $K_{1}(1270)$ and $K_{1}(1400)$, at the scale $\mu=1 \mathrm{GeV} \cdot \Phi_{\perp}^{1} P_{1}(u)$ and $\Phi_{\|}^{3} P_{1}(u)$ are symmetric under $u \leftrightarrow 1-u$ if neglecting $\mathrm{SU}(3)$ breaking effects, whereas $\Phi_{\|}^{1} P_{1}(u)$ and $\Phi_{\perp}^{3} P_{1}(u)$ are antisymmetric.

The contents of $h_{1}(1170)$ and $h_{1}(1380)$ are dominated by their $\bar{u} u$ and $\bar{s} s$ components, respectively, which are slightly different for $\theta_{1_{1}}=10^{\circ}$ and $45^{\circ}$. However, the $\bar{s} s$ component of $h_{1}(1170)$ and $\bar{u} u$ component of $h_{1}(1380)$ become significant for $\theta_{1_{1}}=10^{\circ}$. Analogously, considering the real states $f_{1}(1285)$ and $f_{1}(1420)$, we find that only the $\bar{s} s$ fraction of $f_{1}(1285)$ is a little sensitive to the singlet-octet mixing angle $\theta_{3} P_{1}$ as changing $\theta_{3} P_{1}=38^{\circ}$ to be $50^{\circ}$. In particular, the $\Phi_{\perp}$ and $\Phi_{\|}$for $f_{1}(1285)$ (or for $f_{1}(1420)$ ) are predominated by their $\bar{u} u$ (or $\bar{s} s$ ) component. In Fig. 7(e), it is interesting to note that due to the mixture between $K_{1 A}$ and $K_{1 B}$, where the axial-vector mesons contain an $s$ quark and a light anti-quark $\bar{q}$, we find that the $\bar{q}$ (or $s$ ) carries a larger momentum fraction for the $K_{1}(1270)$ (or $K_{1}(1400)$ ) meson with respect to $\theta=45^{\circ}$. Nevertheless, if $\theta=-45^{\circ}$, the $s$ (or $\bar{q}$ ) instead carries a larger momentum fraction for the $K_{1}(1270)$ (or $K_{1}(1400)$ ) meson.

\subsection{Two-parton LCDAs of twist-three}

Using the equations of motion allows one to rewrite the two-parton LCDAs of twist-three in terms of the leading-twist LCDAs and three-parton LCDAs of twist-3. Thus, substituting the 

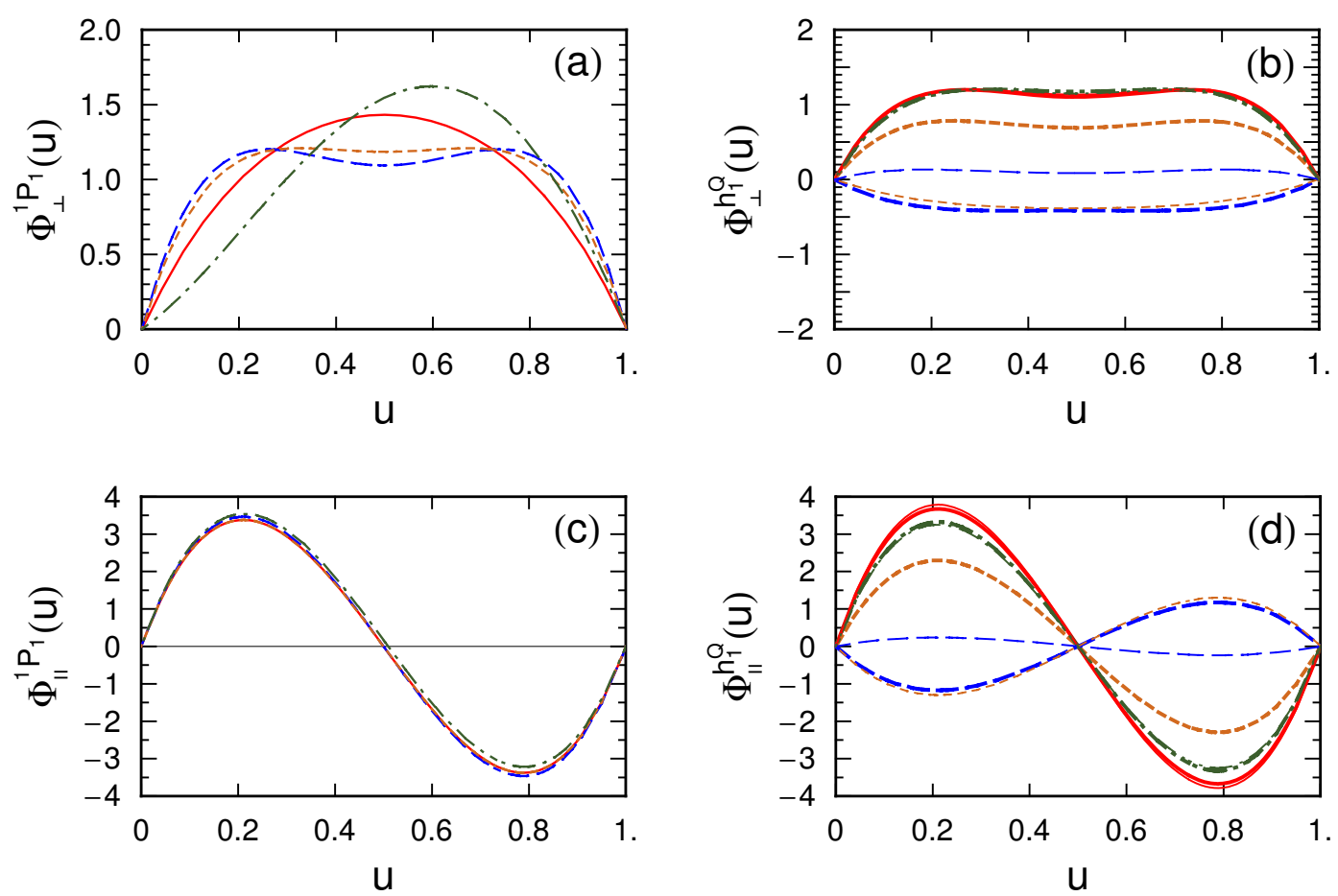

Figure 6: Leading-twist light-cone distribution amplitudes, normalized at the scale $\mu=1 \mathrm{GeV}$, for $1^{1} P_{1}$ states, where the central values of Gegenbauer moments given in Table 4 are used. $u(\bar{u} \equiv 1-u)$ is the meson momentum fraction carried by the quark (antiquark). In (a) and (c), the solid, long-dashed, shortdashed and dot-dashed curves correspond to $b_{1}(1235), h_{1}$ (singlet), $h_{8}$ (octet) and $K_{1 B}$, respectively. In (b) and (d), the solid [short-dashed] and longdashed [dot-dashed] curves correspond to the $\bar{u} u[\bar{s} s]$ contents of $h_{1}(1170)$ and $h_{1}(1380)$, respectively, where $\theta_{1_{1}}=10^{\circ}\left(45^{\circ}\right)$ have been used for heavier (lighter) curves. The definitions for the LCDAs of $h_{1}(1170)$ and $h_{1}(1380)$ have been given in Appendix D.

twist-2 LCDAs specified by Eqs. (245), (246), (249), and (250), and three-parton LCDAs of twist-3 specified by Eqs. (40)-(45) into Eqs. (55), (56), (67), and (68), we get the approximate expressions in linear in quark masses (valid up to conformal spin 9/2):

$$
\begin{aligned}
g_{\perp}^{(a)}(u)= & \frac{3}{4}\left(1+\xi^{2}\right)+\frac{3}{2} a_{1}^{\|} \xi^{3}+\left(\frac{3}{7} a_{2}^{\|}+5 \zeta_{3,{ }^{3} P_{1}}^{V}\right)\left(3 \xi^{2}-1\right) \\
& +\left(\frac{9}{112} a_{2}^{\|}+\frac{105}{16} \zeta_{3,{ }^{3} P_{1}}^{A}-\frac{15}{64} \zeta_{3,{ }^{,} P_{1}}^{V} \omega_{3 P_{1}}^{V}\right)\left(35 \xi^{4}-30 \xi^{2}+3\right) \\
& +5\left[\frac{21}{4} \zeta_{3,{ }^{3} P_{1}}^{V} \sigma_{3 P_{1}}^{V}+\zeta_{3,{ }^{3} P_{1}}^{A}\left(\lambda^{A} P_{1}-\frac{3}{16} \sigma_{3}^{A} P_{1}\right)\right] \xi\left(5 \xi^{2}-3\right) \\
& -\frac{9}{2} \bar{a}_{1}^{\perp} \widetilde{\delta}_{+}\left(\frac{3}{2}+\frac{3}{2} \xi^{2}+\ln u+\ln \bar{u}\right)-\frac{9}{2} \bar{a}_{1}^{\perp} \widetilde{\delta}_{-}(3 \xi+\ln \bar{u}-\ln u),
\end{aligned}
$$



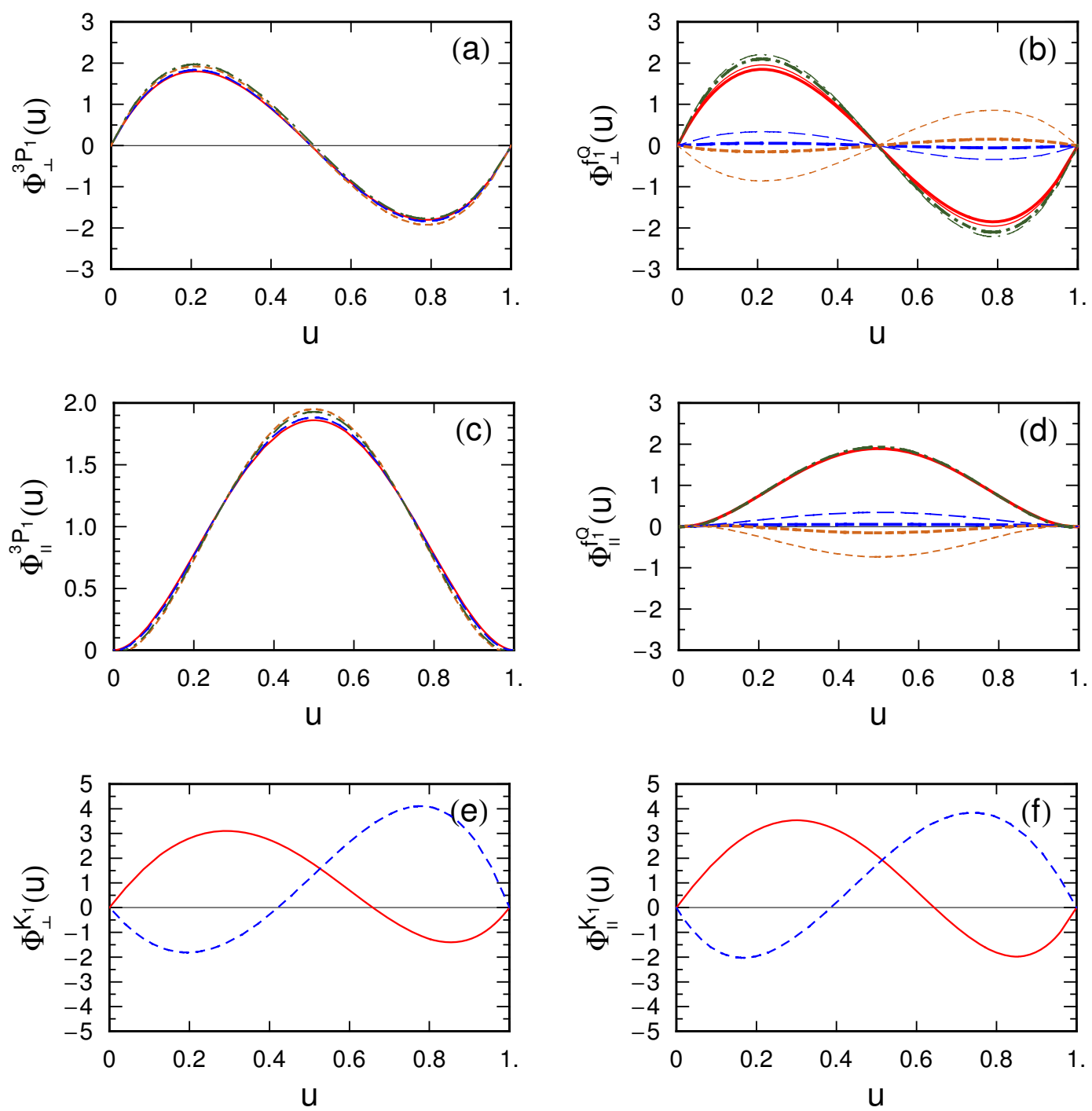

Figure 7: Leading-twist light-cone distribution amplitudes, normalized at the scale $\mu=1 \mathrm{GeV}$, for $1^{3} P_{1}$ states, and for $K_{1}(1270)$ and $K_{1}(1400)$ mesons, where the central values of Gegenbauer moments given in Table 4 are used. $u$ $(\bar{u} \equiv 1-u)$ is the meson momentum fraction carried by the quark (antiquark). In (a) and (c), the solid, long-dashed, short-dashed and dot-dashed curves correspond to $a_{1}(1260), f_{1}$ (singlet), $f_{8}$ (octet) and $K_{1 A}$, respectively. In (b) and (d), the solid [short-dashed] and long-dashed [dot-dashed] curves respectively correspond to the $\bar{u} u[\bar{s} s]$ contents of $f_{1}(1285)$ and $f_{1}(1420)$, where $\theta=38^{\circ}\left(50^{\circ}\right)$ have been used for heavier (lighter) curves. The definitions for the LCDAs of $f_{1}(1285)$ and $f_{1}(1420)$ have been given in Appendix D. In (e) and (f), the solid (dashed) and dashed (solid) curves correspond to $K_{1}(1270)$ and $K_{1}(1400)$, respectively, for $\theta_{K}=45^{\circ}\left(\theta_{K}=-45^{\circ}\right)$.

$g_{\perp}^{(v)}(u)=6 u \bar{u}\left\{1+\left(a_{1}^{\|}+\frac{20}{3} \zeta_{3,{ }^{3} P_{1}}^{A} \lambda_{3}^{A} P_{1}\right) \xi\right.$ 


$$
\begin{aligned}
& +\left[\frac{1}{4} a_{2}^{\|}+\frac{5}{3} \zeta_{3,{ }^{3} P_{1}}^{V}\left(1-\frac{3}{16} \omega^{V} P_{1}\right)+\frac{35}{4} \zeta_{3,{ }^{3} P_{1}}^{A}\right]\left(5 \xi^{2}-1\right) \\
& \left.+\frac{35}{4}\left(\zeta_{3,{ }^{3} P_{1}}^{V} \sigma_{3 P_{1}}^{V}-\frac{1}{28} \zeta_{3,{ }^{3} P_{1}}^{A} \sigma_{3}^{A} P_{1}\right) \xi\left(7 \xi^{2}-3\right)\right\} \\
& -18 \bar{a}_{1}^{\perp} \widetilde{\delta}_{+}(3 u \bar{u}+\bar{u} \ln \bar{u}+u \ln u)-18 \bar{a}_{1}^{\perp} \widetilde{\delta}_{-}(u \bar{u} \xi+\bar{u} \ln \bar{u}-u \ln u), \\
& h_{\|}^{(t)}(u)=3 a_{0}^{\perp} \xi^{2}+\frac{3}{2} a_{1}^{\perp} \xi\left(3 \xi^{2}-1\right)+\frac{3}{2}\left[a_{2}^{\perp} \xi+\zeta_{3,{ }^{3} P_{1}}^{\perp}\left(5-\frac{\omega^{\perp} P_{1}}{2}\right)\right] \xi\left(5 \xi^{2}-3\right) \\
& +\frac{35}{4} \zeta_{3,{ }^{3} P_{1}}^{\perp} \sigma^{\perp} P_{1}\left(35 \xi^{4}-30 \xi^{2}+3\right)+18 \bar{a}_{2}^{\|}\left[\delta_{+} \xi-\frac{5}{8} \delta_{-}\left(3 \xi^{2}-1\right)\right] \\
& -\frac{3}{2}\left(\delta_{+} \xi[2+\ln (\bar{u} u)]+\delta_{-}[1+\xi \ln (\bar{u} / u)]\right)\left(1+6 \bar{a}_{2}^{\|}\right), \\
& h_{\|}^{(p)}(u)=6 u \bar{u}\left\{a_{0}^{\perp}+\left[a_{1}^{\perp}+5 \zeta_{3,{ }^{3} P_{1}}^{\perp}\left(1-\frac{1}{40}\left(7 \xi^{2}-3\right) \omega^{\perp} P_{1}\right)\right] \xi\right. \\
& \left.+\left(\frac{1}{4} a_{2}^{\perp}+\frac{35}{6} \zeta_{3,{ }^{3} P_{1}}^{\perp} \sigma_{3 P_{1}}^{\perp}\right)\left(5 \xi^{2}-1\right)-5 \bar{a}_{2}^{\|}\left[\delta_{+} \xi+\frac{3}{2} \delta_{-}(1-\bar{u} u)\right]\right\} \\
& -3\left[\delta_{+}(\bar{u} \ln \bar{u}-u \ln u)+\delta_{-}(u \bar{u}+\bar{u} \ln \bar{u}+u \ln u)\right]\left(1+6 \bar{a}_{2}^{\|}\right),
\end{aligned}
$$

with the normalization conditions

$$
\begin{aligned}
& \int_{0}^{1} d u g_{\perp}^{(a)}(u)=\int_{0}^{1} d u g_{\perp}^{(v)}(u)=1, \\
& \int_{0}^{1} d u h_{\|}^{(t)}(u)=a_{0}^{\perp}, \\
& \int_{0}^{1} d u h_{\|}^{(p)}(u)=a_{0}^{\perp}+\delta_{-},
\end{aligned}
$$

for pure ${ }^{3} P_{1}$ states, and

$$
\begin{aligned}
g_{\perp}^{(a)}(u)= & \frac{3}{4} a_{0}^{\|}\left(1+\xi^{2}\right)+\frac{3}{2} a_{1}^{\|} \xi^{3}+5\left[\frac{21}{4} \zeta_{3,{ }^{1} P_{1}}^{V}+\zeta_{3,{ }^{1} P_{1}}^{A}\left(1-\frac{3}{16} \omega_{1 P_{1}}^{A}\right)\right] \xi\left(5 \xi^{2}-3\right) \\
& +\frac{3}{16} a_{2}^{\|}\left(15 \xi^{4}-6 \xi^{2}-1\right)+5 \zeta_{3,{ }^{1} P_{1}}^{V} \lambda_{1}^{V} P_{1}\left(3 \xi^{2}-1\right) \\
& +\frac{105}{16}\left(\zeta_{3,{ }^{1} P_{1}}^{A} \sigma_{1 P_{1}}^{A}-\frac{1}{28} \zeta_{{ } P_{1}} \sigma^{V}{ }_{P_{1}}\right)\left(35 \xi^{4}-30 \xi^{2}+3\right) \\
& -15 \bar{a}_{2}^{\perp}\left[\widetilde{\delta}_{+} \xi^{3}+\frac{1}{2} \widetilde{\delta}_{-}\left(3 \xi^{2}-1\right)\right] \\
& -\frac{3}{2}\left[\widetilde{\delta}_{+}(2 \xi+\ln \bar{u}-\ln u)+\widetilde{\delta}_{-}(2+\ln u+\ln \bar{u})\right]\left(1+6 \bar{a}_{2}^{\perp}\right),
\end{aligned}
$$




$$
\begin{aligned}
& g_{\perp}^{(v)}(u)=6 u \bar{u}\left\{a_{0}^{\|}+a_{1}^{\|} \xi+\left[\frac{1}{4} a_{2}^{\|}+\frac{5}{3} \zeta_{3,{ }_{1} P_{1}}^{V}\left(\lambda_{1 P_{1}}^{V}-\frac{3}{16} \sigma_{{ }_{1} P_{1}}^{V}\right)+\frac{35}{4} \zeta_{3,{ }_{1} P_{1}}^{A} \sigma_{1}^{A} P_{1}\right]\left(5 \xi^{2}-1\right)\right. \\
& +\frac{20}{3} \xi\left[\zeta_{3,{ }^{1} P_{1}}^{A}+\frac{21}{16}\left(\zeta_{3,{ }^{1} P_{1}}^{V}-\frac{1}{28} \zeta_{3,{ }^{1} P_{1}}^{A} \omega_{1}^{A} P_{1}\right)\left(7 \xi^{2}-3\right)\right] \\
& \left.-5 \bar{a}_{2}^{\perp}\left[2 \widetilde{\delta}_{+} \xi+\widetilde{\delta}_{-}\left(1+\xi^{2}\right)\right]\right\} \\
& -6\left[\widetilde{\delta}_{+}(\bar{u} \ln \bar{u}-u \ln u)+\widetilde{\delta}_{-}(2 u \bar{u}+\bar{u} \ln \bar{u}+u \ln u)\right]\left(1+6 \bar{a}_{2}^{\perp}\right) \\
& h_{\|}^{(t)}(u)=3 \xi^{2}+\frac{3}{2} a_{1}^{\perp} \xi\left(3 \xi^{2}-1\right)+\left[\frac{3}{2} a_{2}^{\perp} \xi+\frac{15}{2} \zeta_{3,{ }^{1} P_{1}}^{\perp}\left(\lambda^{\perp} P_{1}-\frac{1}{10} \sigma_{{ }^{1} P_{1}}^{\perp}\right)\right] \xi\left(5 \xi^{2}-3\right) \\
& +\frac{35}{4} \zeta_{3,{ }^{1} P_{1}}^{\perp}\left(35 \xi^{4}-30 \xi^{2}+3\right) \\
& +\frac{9}{2} \bar{a}_{1}^{\|} \xi\left[\delta_{+}(\ln u-\ln \bar{u}-3 \xi)-\delta_{-}\left(\ln u+\ln \bar{u}+\frac{8}{3}\right)\right], \\
& h_{\|}^{(p)}(u)=6 u \bar{u}\left\{1+a_{1}^{\perp} \xi+\left(\frac{1}{4} a_{2}^{\perp}+\frac{35}{6} \zeta_{3,{ }^{1} P_{1}}^{\perp}\right)\left(5 \xi^{2}-1\right)\right. \\
& \left.+5 \zeta_{3,{ }^{1} P_{1}}^{\perp}\left[\lambda^{\perp} P_{1}-\frac{1}{40}\left(7 \xi^{3}-3\right) \sigma^{\perp} P_{1}\right] \xi\right\} \\
& -9 \bar{a}_{1}^{\|} \delta_{+}(3 u \bar{u}+\bar{u} \ln \bar{u}+u \ln u)-9 \bar{a}_{1}^{\|} \delta_{-}\left(\frac{2}{3} \xi u \bar{u}+\bar{u} \ln \bar{u}-u \ln u\right),
\end{aligned}
$$

with the normalization conditions

$$
\begin{aligned}
& \int_{0}^{1} d u g_{\perp}^{(a)}(u)=a_{0}^{\|}, \\
& \int_{0}^{1} d u g_{\perp}^{(v)}(u)=a_{0}^{\|}+\widetilde{\delta}_{-}, \\
& \int_{0}^{1} d u h_{\|}^{(t)}(u)=\int_{0}^{1} d u h_{\|}^{(p)}(u)=1,
\end{aligned}
$$

for pure ${ }^{1} P_{1}$ states. Note that to include the corrections consistently in linear in quark masses, in Eqs. (257)-(260) and (264)-(267) the parameters with the "bar" should be replaced by the corresponding ones in the massless quark limit. For the physical $h_{1}(1170), h_{1}(1380), f_{1}(1285)$ and $f_{1}(1420)$ mesons, their two-parton LCDAs of twist-3 are defined in Appendix D. The LCDAs for $K_{1}(1270)$ and $K_{1}(1400)$ are given by

$$
\begin{aligned}
g_{\perp}^{(a, v) K_{1}(1270)} & =\frac{f_{K_{1 A}} m_{K_{1 A}}}{f_{K_{1}(1270)} m_{K_{1}(1270)}} g_{\perp}^{(a, v) K_{1 A}} \sin \theta_{K}+\frac{f_{K_{1 B}} m_{K_{1 B}}}{f_{K_{1}(1270)} m_{K_{1}(1270)}} g_{\perp}^{(a, v) K_{1 B}} \cos \theta_{K}, \\
g_{\perp}^{(a, v) K_{1}(1400)} & =\frac{f_{K_{1 A}} m_{K_{1 A}}}{f_{K_{1}(1400)} m_{K_{1}(1400)}} g_{\perp}^{(a, v) K_{1 A}} \cos \theta_{K}-\frac{f_{K_{1 B}} m_{K_{1 B}}}{f_{K_{1}(1400)} m_{K_{1}(1400)}} g_{\perp}^{(a, v) K_{1 B}} \sin \theta_{K},
\end{aligned}
$$




$$
\begin{aligned}
h_{\|}^{(t, p) K_{1}(1270)} & =\frac{f_{K_{1 A}}^{\perp} m_{K_{1 A}}^{2}}{f_{K_{1}(1270)}^{\perp} m_{K_{1}(1270)}^{2}} h_{\|}^{(t, p) K_{1 A}} \sin \theta_{K}+\frac{f_{K_{1 B}}^{\perp} m_{K_{1 B}}^{2}}{f_{K_{1}(1270)}^{\perp} m_{K_{1}(1270)}^{2}} h_{\|}^{(t, p) K_{1 B}} \cos \theta_{K}, \\
h_{\|}^{(t, p) K_{1}(1400)} & =\frac{f_{K_{1 A}}^{\perp} m_{K_{1 A}}^{2}}{f_{K_{1}(1400)}^{\perp} m_{K_{1}(1400)}^{2}} h_{\|}^{(t, p) K_{1 A}} \cos \theta_{K}-\frac{f_{K_{1 B}}^{\perp} m_{K_{1 B}}^{2}}{f_{K_{1}(1400)}^{\perp} m_{K_{1}(1400)}^{2}} h_{\|}^{(t, p) K_{1 B}} \sin \theta_{K} .
\end{aligned}
$$

Substituting the central values of parameters given in Tables 4, 5, and 6 into the above equations, in Figs. 8 11 we plot two-parton LCDAs of twist-3 at the scale $\mu=1 \mathrm{GeV}$. The properties of twist-3 two-parton LCDAs are analogous to the cases of the leading-twist LCDAs that we have given previously. It is interesting to note again that the LCDAs, $g_{\perp}^{(a)}, g_{\perp}^{(v)}, h_{\|}^{(t)}$, and $h_{\|}^{(p)}$, for $f_{1}(1285)$ (or $f_{1}(1420)$ ) are dominated by the $\bar{u} u$ (or $\bar{s} s$ ) content which is insensitive to singlet-octet mixing angle $\theta_{3} P_{1}$ in the range $38^{\circ}<\theta_{3} P_{1}<50^{\circ}$. Moreover, $g_{\perp}^{(a)}, g_{\perp}^{(v)}, h_{\|}^{(t)}$, and $h_{\|}^{(p)}$ for $h_{1}(1170)$ (or $h_{1}(1380)$ ) are dominated by the $\bar{u} u$ (or $\bar{s} s$ ) content for $\theta_{P_{1}}=45^{\circ}$ but the $\bar{s} s$ (or $\bar{u} u$ ) content becomes significant for $\theta_{1_{1}}=10^{\circ}$.

\subsection{Three-parton LCDAs of twist-three}

The approximate three-parton LCDAs of twist-3 are given in Eqs. (40)-(45) and the relevant parameters are summarized in Tables 5 and 6 . For completeness and simplicity, we plot the LCDAs for the $a_{1}(1260)$ and $b_{1}(1235)$ mesons in Fig. 12 to illustrate their behaviors.

\section{Summary}

The light-cone distribution amplitudes specified by the collinear twist can be expanded in terms of the series of the so-called conformal partial waves. Each partial wave is characterized by a specific conformal spin. For each conformal spin, the dependence of the distribution amplitudes on the transverse coordinates is governed by the renormalization group equation, while the dependence on the longitudinal coordinates is involved in "spherical harmonics" of the $S L(2, \mathbb{R})$ group.

We have presented a detailed study of twist-2 and twist-3 light-cone distribution amplitudes of axial-vector mesons, based on the QCD conformal partial wave expansion. The equations of motion allow us to obtain the relations among the twist-two and twist-three light-cone distribution amplitudes [9], so that we can use a minimal number of independent nonperturbative parameters to describe the distribution amplitudes. The conformal partial wave related by equations of motion should correspond to the same conformal spin since equations of motion in the QCD perturbative theory respect all symmetries given in the classical level.

Our main results are as follows: 

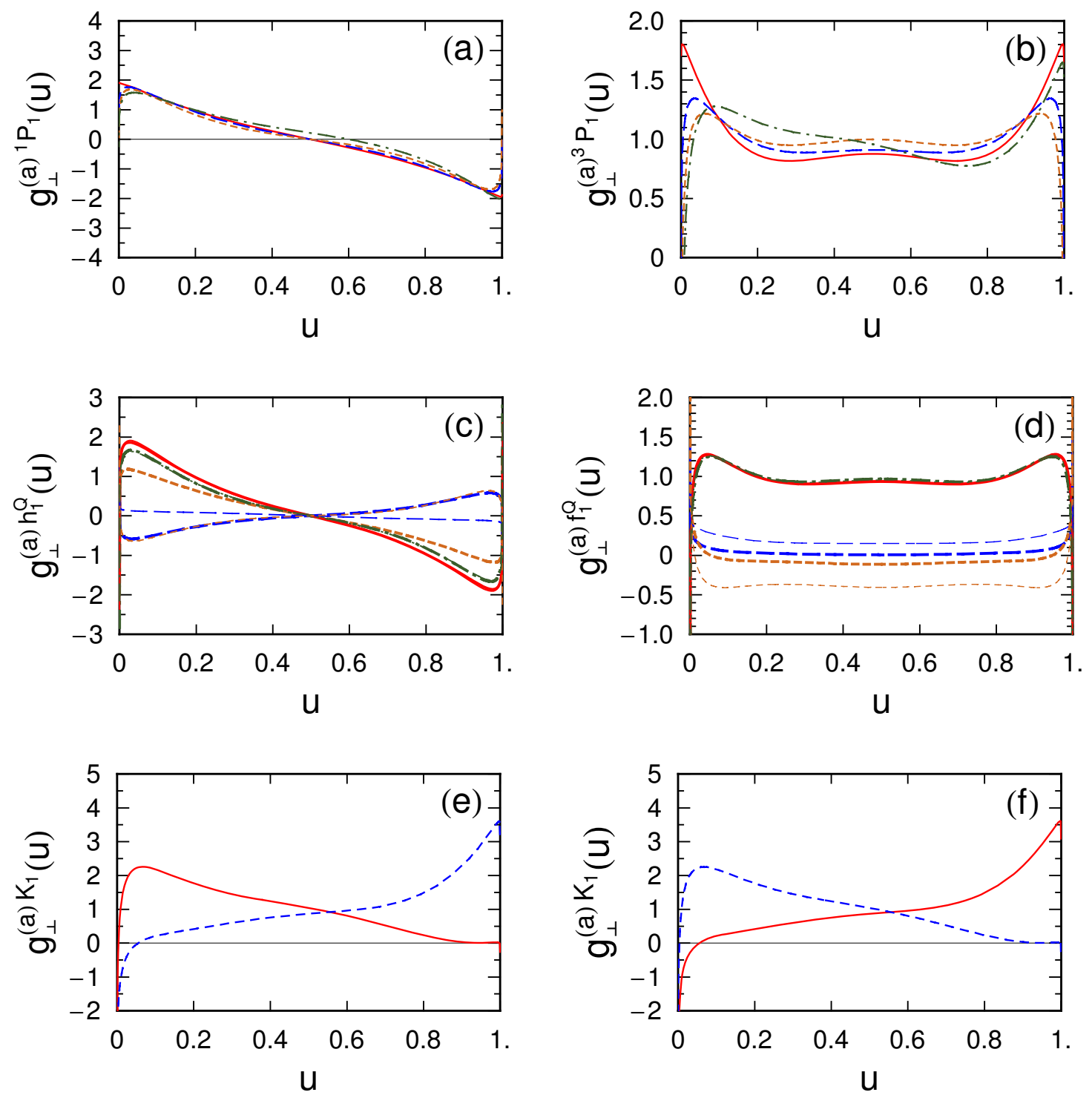

Figure 8: Twist-3 light-cone distribution amplitudes $g_{\perp}^{(a)}$ at the scale $\mu=1 \mathrm{GeV}$, where the central values of parameters given in Tables 4,5 and 6 are used. In (e), where $\theta_{K}=45^{\circ}$, and in (f), where $\theta_{K}=-45^{\circ}$, the solid and dashed curves correspond to $K_{1}(1270)$ and $K_{1}(1400)$, respectively. Others are the same as Figs. [6] and 7 .

- In subsections 4.2 and 4.3 we have shown the sum rule results for the axial-vector (tensor) decay constants of $1^{3} P_{1}\left(1^{1} P_{1}\right)$ axial-vector mesons, where we have updated the values for $1^{1} P_{1}$ states obtained in Ref. [16].

- Using Gell-Mann-Okubo mass formula, we have obtained the mixing angles for the $f_{8}$ (octet) and $f_{1}$ (singlet) of $1^{3} P_{1}$ states to be $\theta^{3} P_{1} \sim 38^{\circ}$, and for $h_{8}$ (octet) and $h_{1}$ (singlet) of $1^{1} P_{1}$ states to be $\theta_{P_{1}} \sim 10^{\circ}$. Thus the decay constants and light-cone 

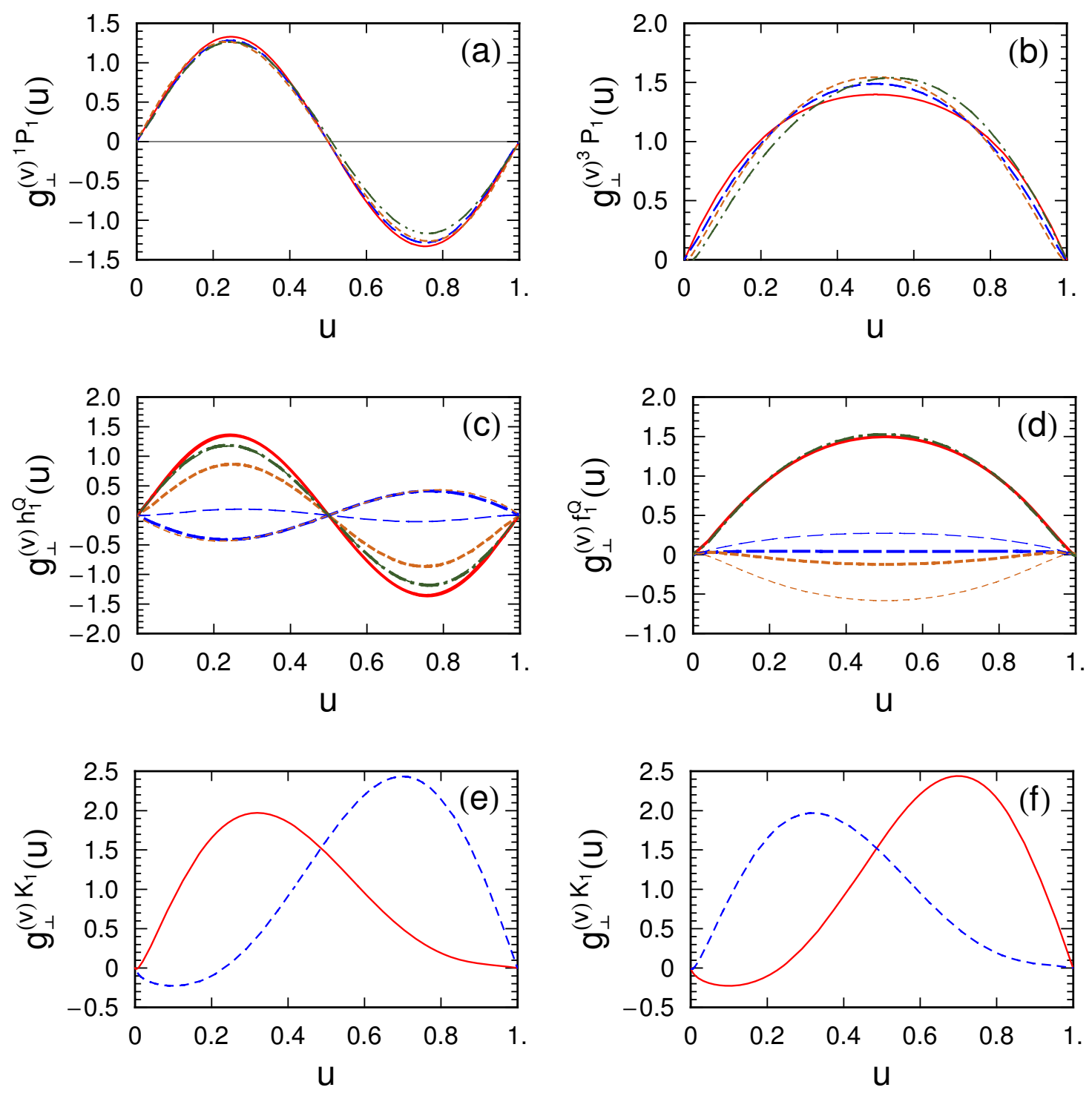

Figure 9: Twist-3 light-cone distribution amplitudes $g_{\perp}^{(v)}$ at the scale $\mu=1 \mathrm{GeV}$. Others are the same as Fig. 8 .

distribution amplitudes for these states are determined.

- The sum rules for the first few Gegenbauer moments of the leading-twist light-cone distribution amplitudes together with their numerical results have been given in subsection 4.4. where the SU(3) breaking effects relevant to the $K_{1 A}$ and $K_{1 B}$ states are included. The results for $1^{3} P_{1}$ states, $h_{1}, h_{8}$ and for G-parity violating Gegenbauer moments are new, while the results of G-parity invariant Gegenbauer moments for $b_{1}$ and $K_{1 B}$ (which are $1^{1} P_{1}$ states) are updated. In the sum rules, because the G-parity violating Gegenbauer moments of $1^{3} P_{1}$ and $1^{1} P_{1}$ are always mixed togther, we thus 

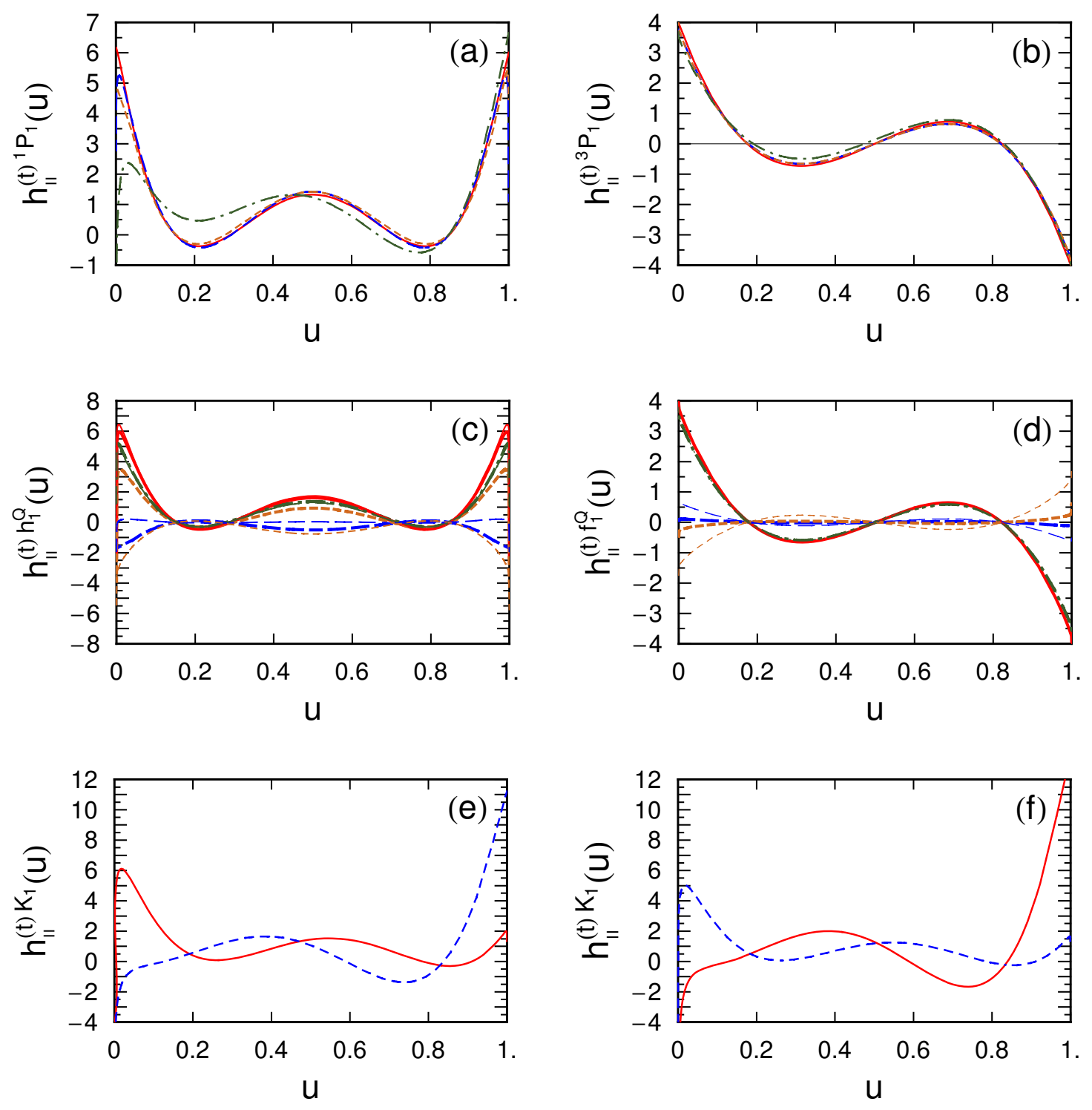

Figure 10: Twist-3 light-cone distribution amplitudes $h_{\|}^{(t)}$ at the scale $\mu=1 \mathrm{GeV}$. Others are the same as Fig. 8 .

add a reasonable constraint

$$
\frac{a_{0}^{\perp, K_{1 A}}}{a_{0}^{\|, K_{1 B}} \frac{m_{K_{1 B}} f_{K_{1 B}} f_{K_{1 B}}^{\perp}}{m_{K_{1 A}} f_{K_{1 A}} f_{K_{1 A}}^{\frac{1}{L_{1 A}}}}}=1.0 \pm 0.3,
$$

which is a good approximation for G-invariant Gegenbauer moments, to obtain qualitative estimates. See the detailed discussions in subsection 4.4.3,

- In Sec. 5, using the QCD sum rules, the relevant G-parity invariant and violating parameters for expanding the three-parton distribution amplitudes of twist-3 in terms 

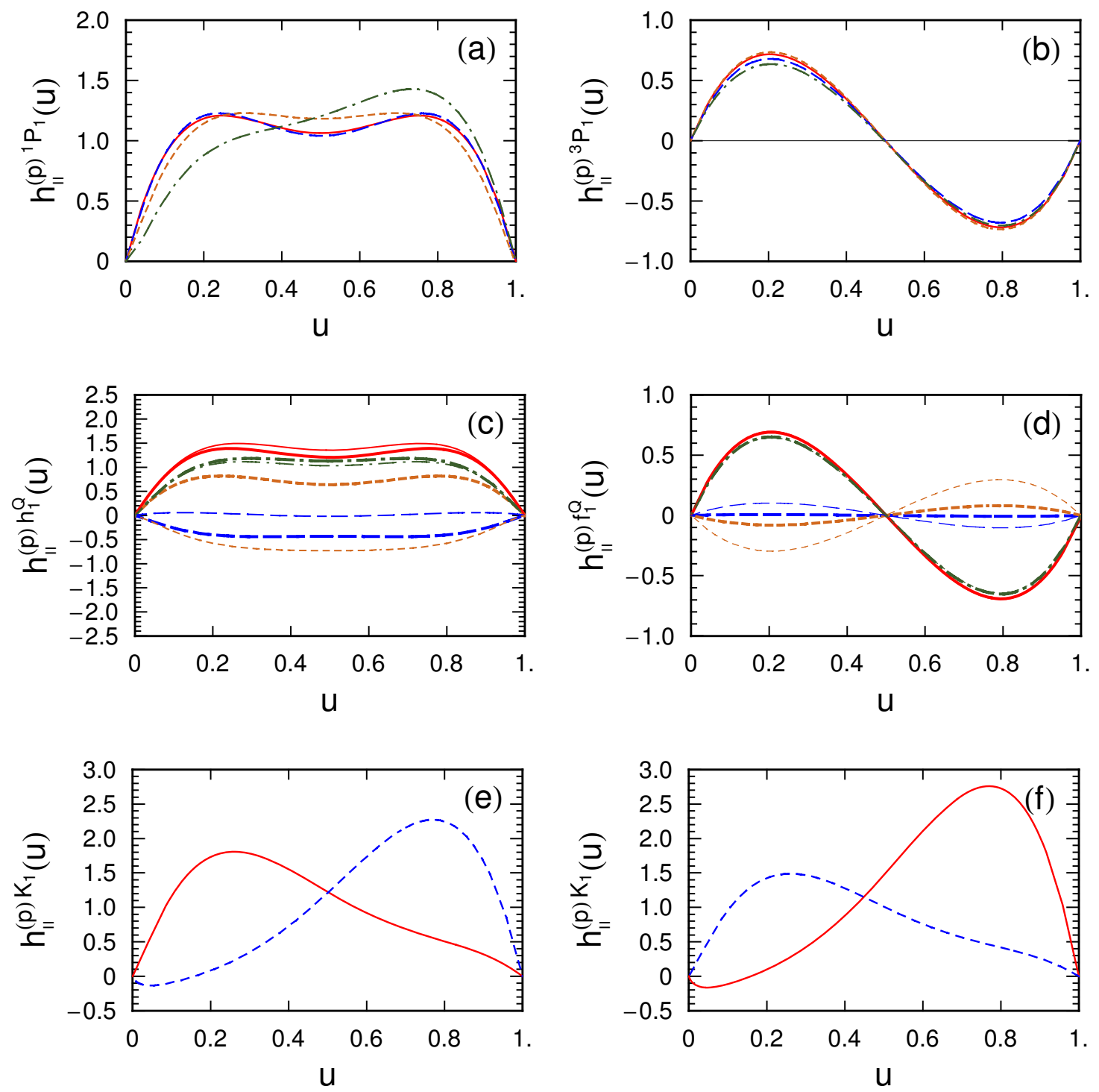

Figure 11: Twist-3 light-cone distribution amplitudes $h_{\|}^{(p)}$ at the scale $\mu=1 \mathrm{GeV}$. Others are the same as Fig. 8 ,

of conformal partial waves with conformal spin up to $9 / 2$ have been evaluated, where the $\mathrm{SU}(3)$ corrections have been contained. To determined not only the magnitudes but also the relative signs for the parameters, one of the interpolating currents in the two-point correlation functions is chosen to be the local axial-vector (or pseudo-tensor) current in calculating the parameters for the $1^{3} P_{1}$ (or $1^{1} P_{1}$ ) state. All the results are new. We have checked all the calculations very carefully since nobody did these before. Only the sum rule calculation for $f_{3,{ }^{1} P_{1}}^{\perp}$ is very similar to that for $f_{3 \rho}^{T}$ in $\mathrm{SU}(3)$ limit (See the discussions after Eq. (244)). On the other hand, the resulting $f_{3,{ }^{3} P 1}^{\perp}$ sum rule is not reliable since the calculated OPE series is not well convergent. We further resort to the diagonal sum rule for $f_{3_{P 1}}^{\perp}$ and this calculation can be found in Ref. [20], 

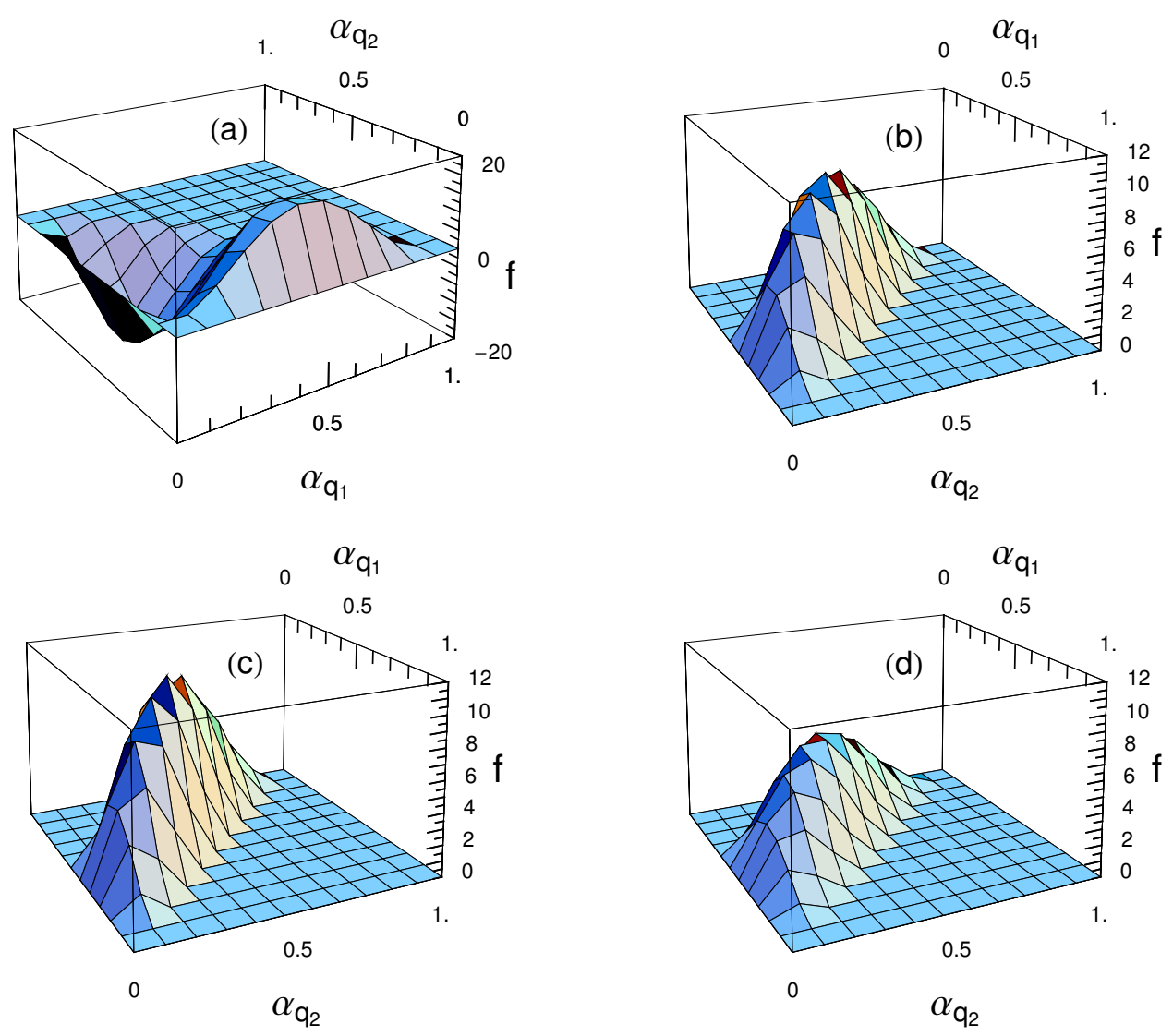

Figure 12: Twist-3 three-parton light-cone distribution amplitudes for the $a_{1}(1260)$ and $b_{1}(1235)$ mesons at the scale $\mu=1 \mathrm{GeV}:$ (a) $f \equiv \mathcal{A}$ for $a_{1}(1260)$ or $f \equiv \mathcal{V}, \mathcal{T}$ for $b_{1}(1235),(\mathrm{b}) f \equiv \mathcal{V}$ for $a_{1}(1260),(\mathrm{c}) f \equiv \mathcal{T}$ for $a_{1}(1260)$, and (d) $f \equiv \mathcal{A}$ for $b_{1}(1235)$, where we have taken the central values of $\omega_{a_{1}}^{\perp}, \omega_{a_{1}}^{V}$, and $\omega_{b_{1}}^{A}$, given in Tables 5 and 6, as inputs. Here $\alpha_{q_{1}}$ and $\alpha_{q_{2}}$ are momentum fractions carried by the quark and anti-quark in an axial-vector meson, respectively. The gluon momentum fraction is substituted as $\alpha_{g}=1-\alpha_{q_{1}}-\alpha_{q_{2}}$.

where the sum rule is used to study the coupling of the kaon. The diagonal sum rule is stable and the sign for $f_{3 P 1}^{\perp}$ can be determined in an indirect way (See Sec. 5.1.3 for more discussions). It should be noted that as G-parity violating parameters for $1^{3} P_{1}$ $\left(1^{1} P_{1}\right)$ states are computed, the corrections receiving from $1^{1} P_{1}\left(1^{3} P_{1}\right)$ states have to be considered.

- Adapting the EOM formulas derived in Ref. [9] for vector mesons to the present case, the two-parton LCDAs of twist-three can be written in terms of the leading-twist LCDAs and three-parton LCDAs of twist-3. The detailed results for twist-three threeparton LCDAs are shown in Sec. 6.2. In the $\mathrm{SU}(3)$ limit, the detailed symmetric properties of LCDAs of axial-vector mesons, as compared with that of the vector mesons, are summarized in Table 1 . 
- Using the conformal partial expansion, we presented the models for light-cone distribution amplitudes, containing contributions with conformal spin up to 9/2, in Sec. 6.

- We have considered the strange quark mass corrections to distribution amplitudes for the strange axial-vector mesons, $K_{1}(1270)$ and $K_{1}(1400)$. It is interesting to note that $\Phi_{\perp}^{3} P_{1}(u), \Phi_{\|}^{1} P_{1}(u), g_{\perp}^{(a)^{1} P_{1}}, g_{\perp}^{(v)^{1} P_{1}}, h_{\|}^{(t)^{3} P_{1}}, h_{\|}^{(p)^{3} P_{1}}$ have significant antisymmetric behaviors, which should be phenomenologically attractive.

It should be noted that because corrections due the higher resonance and radiative correction in OPE may partially cancel each other for non-diagonal sum rules, it is estimated that the resultant errors for parameters of LCDAs may be $\sim 10 \%$. On the other hand, because we do not calculate the radiative corrections to the perturbative term in diagonal sum rules, these corrections may also lead to $\sim 10 \%$ errors for results. However, since we do not do the qualitative calculations about these effects, we thus do not include this possible error in Tables 4, 5, and 6.

Recently, Belle has measured $B^{-} \rightarrow K_{1}^{-}(1270) \gamma$ and given an upper bound on $B^{-} \rightarrow$ $K_{1}^{-}(1400) \gamma$ [11]. Interestingly, the recent calculations [43, 44] of adopting LCSR (light-cone sum rule) form factors [45] gave too small predictions for $\mathcal{B}\left(B^{-} \rightarrow K_{1}^{-}(1270) \gamma\right)$ as compared with the data. Since the physical states $K_{1}(1270)$ and $K_{1}(1400)$ are the mixture of $K_{1 A}$ and $K_{1 B}$ which are respectively the pure $1^{3} P_{1}$ and $1^{1} P_{1}$ states, the light-cone distribution amplitudes of $K_{1 A}$ and $K_{1 B}$ are relevant to the results of $B \rightarrow K_{1}(1270)$ and $K_{1}(1400)$ transition form factors. It is known that for $K_{1 B}, \Phi_{\|}$is antisymmetric, while $\Phi_{\perp}$ is symmetric in the $\mathrm{SU}(3)$ limit due to G-parity. Nevertheless, for $K_{1 A}, \Phi_{\|}$becomes symmetric, while $\Phi_{\perp}$ is antisymmetric. The above properties were not correctly studied in the literature. Some related researches will be published elsewhere [17.

\section{Acknowledgments}

This work was supported in part by the National Science Council of R.O.C. under Grant No: NSC94-2112-M-033-001. 


\section{Appendices}

\section{A Spin Projections and Collinear Twist}

Considering infinitesimal rotation $x_{\mu} \rightarrow x_{\mu}^{\prime}=x_{\mu}+\epsilon_{\mu \nu} x^{\nu}$ in the four dimension, the general field $\Phi(x)$ transforms as $\Phi^{\prime}\left(x^{\prime}\right)=\left[1-\epsilon_{\mu \nu}\left(x^{\mu} \partial^{\nu}-x^{\nu} \partial^{\mu}-\Sigma^{\mu \nu}\right)\right] \Phi(x)=\left[1+i \epsilon_{\mu \nu} M^{\mu \nu}\right] \Phi(x)$, where $\Sigma^{\mu \nu}$ is called the generator of spin rotations of the field $\Phi$. For scalar, quark and gluon fields, we have

$$
\Sigma^{\mu \nu} \phi(x)=0, \quad \Sigma^{\mu \nu} \psi=\frac{i}{2} \sigma^{\mu \nu} \psi, \quad \Sigma^{\mu \nu} A^{\alpha}=g^{\nu \alpha} A^{\mu}-g^{\mu \alpha} A^{\nu},
$$

respectively, where $A_{\mu} \equiv T^{a} A_{\mu}^{a}$. We can apply the following spin projections

$$
P_{+}=\frac{1}{2} \gamma_{-} \gamma_{+}, \quad P_{-}=\frac{1}{2} \gamma_{+} \gamma_{-}, \quad P_{+}+P_{-}=1
$$

on a quark field $\psi$ to project its typical spin component, so that a quark field with a given spin component $s$ on the moving direction can be measured to be

$$
\Sigma_{+-} P_{ \pm} \psi=s_{ \pm} P_{ \pm} \psi= \pm \frac{1}{2} P_{ \pm} \psi
$$

Recalling that the canonical dimension 10 of a quark field is $\ell=3 / 2$, we can therefore decompose an arbitrary light-ray quark-antiquark current into different (collinear) twists $(=$ dimension - spin projection on the moving direction) components:

$$
\begin{aligned}
\bar{\psi}_{2}\left(x_{2-}\right) \Gamma \psi_{1}\left(x_{1-}\right)= & \underbrace{\bar{\psi}_{2}\left(x_{2-}\right) P_{-} \Gamma P_{+} \psi_{1}\left(x_{1-}\right)}_{\text {twist- } 2} \\
& +\underbrace{\bar{\psi}_{2}\left(x_{2-}\right) P_{+} \Gamma P_{+} \psi_{1}\left(x_{1-}\right)+\bar{\psi}_{2}\left(x_{2-}\right) P_{-} \Gamma P_{-} \psi_{1}\left(x_{1-}\right)}_{\text {twist-3 }} \\
& +\underbrace{\bar{\psi}_{2}\left(x_{2-}\right) P_{+} \Gamma P_{-} \psi_{1}\left(x_{1-}\right)}_{\text {twist-4 }},
\end{aligned}
$$

where $\Gamma$ stands for a generic Dirac matrix structure. Note that, taking $\bar{\psi}_{2}\left(x_{2-}\right) P_{-} \Gamma P_{+} \psi_{1}\left(x_{1-}\right)$ as an example, its (collinear) twist exactly equals to $t=\ell_{1}+\ell_{2}-s_{1}-s_{2}=2$, while its conformal spin is $j=\left(\ell_{1}+\ell_{2}+s_{1}+s_{2}+n\right) / 2=j_{1}+j_{2}+n$ with $n=0,1,2, \cdots$; in other words, $j$ is not a fixed value for a LCDA defined by a non-local composite field as seen in Eq. (13). On the other hand, for a gluon field with the canonical dimension $\ell=2$, one can find that

$$
\begin{aligned}
& \Sigma_{+-} G_{+\perp}=1 \cdot G_{+\perp}, \\
& \Sigma_{+-} G_{\perp \perp}=0 \cdot G_{\perp \perp}, \quad \Sigma_{+-} G_{+-}=0 \cdot G_{+-}, \\
& \Sigma_{+-} G_{-\perp}=-1 \cdot G_{-\perp},
\end{aligned}
$$

\footnotetext{
${ }^{10}$ Here we do not distinguish the canonical dimension $\ell^{\text {can }}$ and scaling dimension $\ell$.
} 
where, for instance, $G_{+\perp} \equiv G_{\mu \nu} n^{\mu} g_{\perp}^{\nu \alpha}$ and $g_{\perp}^{\nu \alpha}=g^{\nu \alpha}-n^{\nu} \bar{n}^{\alpha}-n^{\alpha} \bar{n}^{\nu}$. In analogy to the previous discussion, we can decompose an arbitrary light-ray quark-gluon-antiquark current into currents with different twists:

$$
\begin{aligned}
& \bar{\psi}_{2}\left(x_{2-}\right) \Gamma g_{s} G_{\mu \nu}\left(x_{3-}\right) \psi_{1}\left(x_{1-}\right)=\underbrace{\bar{\psi}_{2}\left(x_{2-}\right) P_{-} \Gamma g_{s} G_{\alpha \beta}\left(x_{3-}\right) P_{+} \psi_{1}\left(x_{1-}\right)\left(g_{\perp}^{\alpha \mu} n^{\beta} \bar{n}^{\nu}+n^{\alpha} \bar{n}^{\mu} g_{\perp}^{\beta \nu}\right)}_{\text {twist-3 }} \\
& +\underbrace{\bar{\psi}_{2}\left(x_{2-}\right) P_{+} \Gamma g_{s} G_{\alpha \beta}\left(x_{3-}\right) P_{+} \psi_{1}\left(x_{1-}\right)\left(g_{\perp}^{\alpha \mu} n^{\beta} \bar{n}^{\nu}+n^{\alpha} \bar{n}^{\mu} g_{\perp}^{\beta \nu}\right)}_{\text {twist-4 }} \\
& +\underbrace{\bar{\psi}_{2}\left(x_{2-}\right) P_{-} \Gamma g_{s} G_{\alpha \beta}\left(x_{3-}\right) P_{-} \psi_{1}\left(x_{1-}\right)\left(g_{\perp}^{\alpha \mu} n^{\beta} \bar{n}^{\nu}+n^{\alpha} \bar{n}^{\mu} g_{\perp}^{\beta \nu}\right)}_{\text {twist-4 }} \\
& +\underbrace{\bar{\psi}_{2}\left(x_{2-}\right) P_{-} \Gamma g_{s} G_{\alpha \beta}\left(x_{3-}\right) P_{+} \psi_{1}\left(x_{1-}\right)\left(g_{\perp}^{\alpha \mu} g_{\perp}^{\beta \nu}+n^{\alpha} \bar{n}^{\mu} \bar{n}^{\beta} n^{\nu}+\bar{n}^{\alpha} n^{\mu} n^{\beta} \bar{n}^{\nu}\right)}_{\text {twist- } 4} \\
& +\mathcal{O}(\text { twist-5,6,7). }
\end{aligned}
$$

\section{B Operator Identities}

The operator identities, which are used to obtain the integral equations, Eqs. (52), (53), (64), and (65), are as follows:

$$
\begin{aligned}
\bar{q}_{2}(x) \gamma_{\mu} q_{1}(-x)= & \int_{0}^{1} d t \frac{\partial}{\partial x^{\mu}} \bar{q}_{2}(t x) \not q_{1}(-t x) \\
& +\int_{0}^{1} d t t \int_{-t}^{t} d v \bar{q}_{2}(t x) g_{s} \tilde{G}_{\mu \nu}(v x) x^{\nu} \not x \gamma_{5} q_{1}(-t x) \\
& +i \int_{0}^{1} d t \int_{-t}^{t} d v v \bar{q}_{2}(t x) g_{s} G_{\mu \nu}(v x) x^{\nu} \not x q_{1}(-t x) \\
& -i \epsilon_{\mu \nu \alpha \beta} \int_{0}^{1} d t t x^{\nu} \partial^{\alpha}\left[\bar{q}_{2}(t x) \gamma^{\beta} \gamma_{5} q_{1}(-t x)\right] \\
& +\left(m_{q_{2}}-m_{q_{1}}\right) x^{\nu} \int_{0}^{1} d t t \bar{q}_{2}(t x) \sigma_{\nu \mu} q_{1}(-t x) \\
\bar{q}_{2}(x) \gamma_{\mu} \gamma_{5} q_{1}(-x)= & \int_{0}^{1} d t \frac{\partial}{\partial x^{\mu}} \bar{q}_{2}(t x) \not x \gamma_{5} q_{1}(-t x) \\
& +\int_{0}^{1} d t t \int_{-t}^{t} d v \bar{q}_{2}(t x) g_{s} \tilde{G}_{\mu \nu}(v x) x^{\nu} \not x q_{1}(-t x) \\
& +i \int_{0}^{1} d t \int_{-t}^{t} d v v \bar{q}_{2}(t x) g_{s} G_{\mu \nu}(v x) x^{\nu} \not x \gamma_{5} q_{1}(-t x)
\end{aligned}
$$




$$
\begin{gathered}
-i \epsilon_{\mu \nu \alpha \beta} \int_{0}^{1} d t t x^{\nu} \partial^{\alpha}\left[\bar{q}_{2}(t x) \gamma^{\beta} q_{1}(-t x)\right] \\
+\left(m_{q_{2}}+m_{q_{1}}\right) x^{\nu} \int_{0}^{1} d t t \bar{q}_{2}(t x) \sigma_{\nu \mu} \gamma_{5} q_{1}(-t x) \\
\frac{\partial}{\partial x_{\mu}} \bar{q}_{2}(x) \sigma_{\mu \nu} \gamma_{5} q_{1}(-x)=-i \partial_{\nu} \bar{q}_{2}(x) \gamma_{5} q_{1}(-x)-\int_{-1}^{1} d v \bar{q}_{2}(x) x^{\alpha} g_{s} G_{\alpha \nu}(v x) \gamma_{5} q_{1}(-x) \\
+i \int_{-1}^{1} d v \bar{q}_{2}(x) x_{\rho} g_{s} G^{\rho \mu}(v x) \sigma_{\mu \nu} \gamma_{5} q_{1}(-x) \\
-\left(m_{q_{2}}+m_{q_{1}}\right) \bar{q}_{2}(x) \gamma_{\nu} \gamma_{5} q_{1}(-x) \\
\bar{q}_{2}(x) \gamma_{5} q_{1}(-x)-\bar{q}_{2}(0) \gamma_{5} q_{1}(0)=-\int_{0}^{1} d t \int_{-t}^{t} d v \bar{q}_{2}(t x) x^{\alpha} \sigma_{\alpha \beta} x_{\mu} g_{s} G^{\mu \beta}(v x) \gamma_{5} q_{1}(-t x) \\
+i \int_{0}^{1} d t \partial^{\alpha}\left\{\bar{q}_{2}(t x) \sigma_{\alpha \beta} x^{\beta} \gamma_{5} q_{1}(-t x)\right\} \\
+i\left(m_{q_{2}}-m_{q_{1}}\right) \int_{0}^{1} d t \bar{q}_{2}(t x) \not x \gamma_{5} q_{1}(-t x)
\end{gathered}
$$

where we have adopted the following notation to stand for the total derivative:

$$
\left.\partial_{\mu}\left\{\bar{q}_{2}(x) \Gamma q_{1}(-x)\right\} \equiv \frac{\partial}{\partial y_{\mu}}\left\{\bar{q}_{2}(x+y)[x+y,-x+y] \Gamma q_{1}(-x+y)\right\}\right|_{y \rightarrow 0} .
$$

Eqs. (B.1), (B.2), and ( $(\overline{B .3})$ have been obtained in Refs. [41, 9], whereas Eq. (B.4 $)$ is new.

\section{Input parameters}

The theoretical input parameters, used in our analysis, together with their respective ranges of uncertainty are summarized here. We take into account $\alpha_{s}(1 \mathrm{GeV})=0.497 \pm 0.005$, corresponding to the world average $\alpha_{s}\left(m_{Z}\right)=0.1176 \pm 0.0020$ [28], and the following relevant parameters at the scale $\mu=1 \mathrm{GeV}$ [46, 40, 20, 39]:

$$
\begin{array}{ll}
\left\langle\alpha_{s} G_{\mu \nu}^{a} G^{a \mu \nu}\right\rangle=(0.474 \pm 0.120) \mathrm{GeV}^{4} /(4 \pi), & \\
\langle\bar{u} u\rangle \cong\langle\bar{d} d\rangle=-(0.24 \pm 0.010)^{3} \mathrm{GeV}^{3}, & \langle\bar{s} s\rangle=(0.8 \pm 0.1)\langle\bar{u} u\rangle, \\
\left(m_{u}+m_{d}\right) / 2=(5 \pm 2) \mathrm{MeV}, & m_{s}=(140 \pm 20) \mathrm{MeV}, \\
\left\langle g_{s} \bar{u} \sigma G u\right\rangle \cong\left\langle g_{s} \bar{d} \sigma G d\right\rangle=-(0.8 \pm 0.1)\langle\bar{u} u\rangle, & \left\langle g_{s} \bar{s} \sigma G s\right\rangle=(0.8 \pm 0.1)\left\langle g_{s} \bar{u} \sigma G u\right\rangle, \\
f_{3 P S}=(0.0045 \pm 0.0015) \mathrm{GeV}^{2}, & \\
s_{0}^{\pi}=0.8 \mathrm{GeV}^{2}, & s_{0}^{f_{1}} \simeq s_{0}^{f_{8}} \simeq s_{0}^{K}=1.1 \mathrm{GeV}^{2}, \\
a_{1}^{\perp, K^{*}}=0.04 \pm 0.03, & f_{K^{*}}^{\perp}=(185 \pm 10) \mathrm{MeV}, \\
a_{2}^{\perp, \rho}=0.2 \pm 0.1, & a_{2}^{\perp, \phi}=0.0 \pm 0.1, \\
a_{2}^{\perp, K^{*}}=0.13 \pm 0.08 . &
\end{array}
$$


where the scale-dependence of operators is given by [46]:

$$
\begin{aligned}
& m_{q}(Q)=m_{q}(\mu)\left(\frac{\alpha_{s}(Q)}{\alpha_{s}(\mu)}\right)^{4 / b}, \\
& \langle\bar{q} q\rangle(Q)=\langle\bar{q} q\rangle(\mu)\left(\frac{\alpha_{s}(Q)}{\alpha_{s}(\mu)}\right)^{-4 / b}, \\
& \left\langle g_{s} \bar{q} \sigma \cdot G q\right\rangle(Q)=\left\langle g_{s} \bar{q} \sigma \cdot G q\right\rangle(\mu)\left(\frac{\alpha_{s}(Q)}{\alpha_{s}(\mu)}\right)^{2 /(3 b)}, \\
& \left\langle\alpha_{s} G^{2}\right\rangle(Q)=\left\langle\alpha_{s} G^{2}\right\rangle(\mu),
\end{aligned}
$$

with $b=\left(11 N_{c}-2 n_{f}\right) / 3$. As described in Eq. (86), we adopt the vacuum saturation approximation for describing the four-quark condensates, i.e.,

$$
\left\langle 0\left|\bar{q} \Gamma_{i} \lambda^{a} q \bar{q} \Gamma_{i} \lambda^{a} q\right| 0\right\rangle=-\frac{1}{16 N_{c}^{2}} \operatorname{Tr}\left(\Gamma_{i} \Gamma_{i}\right) \operatorname{Tr}\left(\lambda^{a} \lambda^{a}\right)\langle\bar{q} q\rangle^{2} .
$$

Performing the analysis in analogy to that given in Ref. 9] and using the results in Refs. [47, [48, 49] and in Eqs. (187), (188), (189), (208), (209), (210), (224), (225), (226), we obtain the LO scale-dependence of the twist-3 parameters with the light quark mass corrections as below

[G-parity invariant components]:

$$
\begin{aligned}
& f_{3,{ }^{3} P_{1}}^{V}(Q)=L^{\Gamma_{2}^{+} / b} f_{3,{ }^{3} P_{1}}^{V}(\mu), \quad \Gamma_{2}^{+}=-\frac{1}{3} C_{F}+3 C_{G}, \\
& \left(\begin{array}{l}
f_{3,{ }^{3} P_{1}}^{V} \omega^{V} P_{1}+\frac{28}{3} f_{3,{ }^{3} P_{1}}^{A} \\
f_{3,{ }^{3} P_{1}}^{V} \omega_{3 P_{1}}^{V}-\frac{28}{3} f_{3,{ }^{3} P_{1}}^{A}
\end{array}\right)_{Q}=L^{\Gamma_{3}^{-} / b}\left(\begin{array}{c}
f_{3,{ }^{3} P_{1}}^{V} \omega_{3 P_{1}}^{V}+\frac{28}{3} f_{3,{ }^{3} P_{1}}^{A} \\
f_{3,{ }^{3} P_{1}{ }^{V} \omega_{3} P_{1}}^{V}-\frac{28}{3} f_{3,{ }^{3} P_{1}}^{A}
\end{array}\right)_{\mu}, \\
& \Gamma_{3}^{-}=\left(\begin{array}{ll}
\frac{1}{6} C_{F}+4 C_{G} & \frac{5}{3} C_{F}-\frac{4}{3} C_{G} \\
\frac{2}{3} C_{F}-\frac{2}{3} C_{G} & \frac{8}{3} C_{F}+\frac{7}{3} C_{G}
\end{array}\right), \\
& f_{3,{ }^{3} P_{1}}^{\perp}(Q)=L^{\Gamma^{T_{2}^{+}} / b} f_{3,{ }^{3} P_{1}}^{\perp}(\mu)-\frac{4}{19}\left(L^{4 / b}-L^{\Gamma^{T_{2}^{+}} / b}\right)\left(m_{q_{1}}+m_{q_{2}}\right)(\mu) f_{3} P_{1}, \\
& \Gamma^{T_{2}^{+}}=\frac{7}{3} C_{F}+C_{G},
\end{aligned}
$$

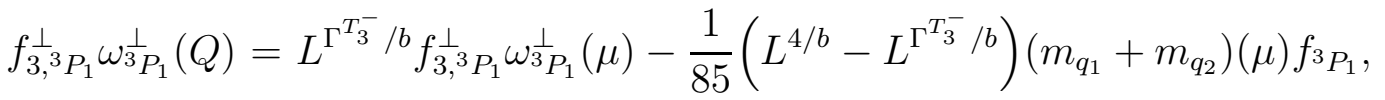

$$
\begin{aligned}
& \Gamma^{T_{3}^{-}}=\frac{7}{6} C_{F}+\frac{10}{3} C_{G}
\end{aligned}
$$


[G-parity violating components]:

$$
\begin{aligned}
& f_{3,{ }^{3} P_{1}}^{A} \lambda_{3}^{A} P_{1}(Q)=L^{\Gamma_{2}^{-} / b} f_{3,{ }^{3} P_{1}}^{A} \lambda_{3 P_{1}}^{A}(\mu), \quad \Gamma_{2}^{-}=-\frac{1}{3} C_{F}+3 C_{G}, \\
& \left(\begin{array}{c}
\frac{28}{3} f_{3,{ }^{3} P_{1}}^{V} \sigma_{3 P_{1}}^{V}-f_{3,{ }^{3} P_{1}}^{A} \sigma_{3 P_{1}}^{A} \\
\frac{28}{3} f_{3,{ }_{3} P_{1}}^{V} \sigma_{3 P_{1}}^{V}+f_{3,{ }_{3} P_{1}}^{A} \sigma_{3 P_{1}}^{A}
\end{array}\right)_{Q}=L^{\Gamma_{3}^{+} / b}\left(\begin{array}{c}
\frac{28}{3} f_{3,{ }^{3} P_{1}}^{V} \sigma_{3 P_{1}}^{V}-f_{3,{ }^{3} P_{1}}^{A} \sigma_{3 P_{1}}^{A} \\
\frac{28}{3} f_{3,{ }_{3} P_{1}}^{V} \sigma_{3 P_{1}}^{V}+f_{3,{ }_{3} P_{1}}^{A} \sigma_{3 P_{1}}^{A}
\end{array}\right)_{\mu}, \\
& \Gamma_{3}^{+}=\left(\begin{array}{cc}
\frac{8}{3} C_{F}+\frac{7}{3} C_{G} & \frac{2}{3} C_{F}-\frac{2}{3} C_{G} \\
\frac{5}{3} C_{F}-\frac{4}{3} C_{G} & \frac{1}{6} C_{F}+4 C_{G}
\end{array}\right), \\
& f_{3,{ }^{3} P_{1}}^{\perp} \sigma_{3 P_{1}}^{\perp}(Q)=L^{\Gamma^{T_{3}^{+}} / b} f_{3,{ }^{3} P_{1}}^{\perp} \sigma_{3}^{\perp} P_{1}(\mu)+\frac{1}{37}\left(L^{4 / b}-L^{\Gamma^{T_{3}^{+}} / b}\right)\left(m_{q_{1}}-m_{q_{2}}\right)(\mu) f_{3} P_{1}, \\
& \Gamma^{T_{3}^{+}}=\frac{23}{6} C_{F}+C_{G},
\end{aligned}
$$

for $1^{3} P_{1}$ states, and

[G-parity invariant components]:

$$
\begin{aligned}
& f_{3,{ }^{1} P_{1}}^{A}(Q)=L^{\Gamma_{2}^{-} / b} f_{3,{ }_{1} P_{1}}^{A}(\mu)-\frac{2}{29}\left(L^{16 /(3 b)}-L^{\Gamma_{2}^{-} / b}\right)\left(m_{q_{1}}+m_{q_{2}}\right)(\mu) f_{{ }_{1} P_{1}}^{\perp}(\mu), \\
& \left(\begin{array}{l}
\frac{28}{3} f_{3,1}^{V}-f_{31}^{A}{ }^{1} P_{1} \omega_{1 P_{1}}^{A} \\
\frac{28}{3} f_{3,{ }_{1} P_{1}}^{V}+f_{3,{ }_{1} P_{1}}^{A} \omega_{1 P_{1}}^{A}
\end{array}\right)_{Q}=L^{\Gamma_{3}^{+} / b}\left(\begin{array}{c}
\frac{28}{3} f_{3,{ }^{1} P_{1}}^{V}-f_{3,{ }^{3} P_{1}}^{A} \omega_{1 P_{1}}^{A} \\
\frac{28}{3} f_{3,{ }^{1} P_{1}}^{V}+f_{3,{ }^{3} P_{1}}^{A} \omega_{1 P_{1}}^{A}
\end{array}\right)_{\mu} \\
& -\frac{8}{795}\left(\begin{array}{c}
L^{16 /(3 b)}-L^{560 /(9 b)} \\
-L^{16 /(3 b)}+L^{-560 /(9 b)}
\end{array}\right)\left(m_{q_{1}}+m_{q_{2}}\right)(\mu) f_{1_{P_{1}}}^{\perp}(\mu), \\
& f_{3,{ }^{1} P_{1}}^{\perp}(Q)=L^{\Gamma^{T_{3}^{+}} / b} f_{3,{ }_{1} P_{1}}^{\perp}(\mu),
\end{aligned}
$$

[G-parity violating components]:

$$
\begin{aligned}
& f_{3,{ }_{1} P_{1}}^{V} \lambda_{1_{P_{1}}}^{V}(Q)=L^{\Gamma_{2}^{+} / b} f_{3,{ }_{1}{ }_{1}}^{V} \lambda_{1}^{V} P_{1}(\mu)+\frac{2}{29}\left(L^{16 /(3 b)}-L^{\Gamma_{2}^{+} / b}\right)\left(m_{q_{1}}-m_{q_{2}}\right)(\mu) f_{{ }_{1} P_{1}}^{\perp}(\mu),
\end{aligned}
$$

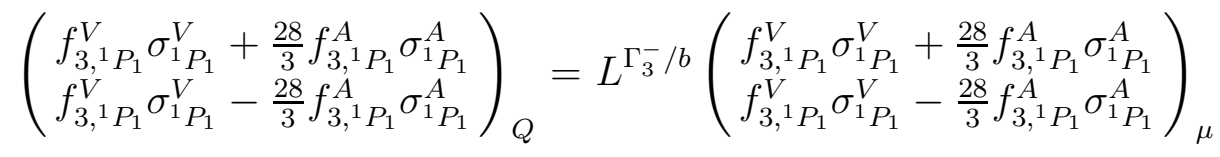

$$
\begin{aligned}
& -\frac{59}{7080}\left(\begin{array}{c}
L^{16 /(3 b)}-L^{-896 /(9 b)} \\
L^{16 /(3 b)}-L^{896 /(9 b)}
\end{array}\right)\left(m_{q_{1}}-m_{q_{2}}\right)(\mu) f_{1_{1}}^{\perp}(\mu), \\
& f_{3,{ }^{1} P_{1}}^{\perp} \lambda_{{ }^{1}}^{\perp} P_{1}(Q)=L^{\Gamma^{T_{2}^{+}} / b} f_{3,{ }_{1} P_{1}}^{\perp} \lambda_{{ }_{1}}^{\perp}(\mu), \\
& f_{3,{ }_{1} P_{1}}^{\perp} \sigma_{1 P_{1}}^{\perp}(Q)=L^{\Gamma^{T_{3}^{-}} / b} f_{3,{ }^{1} P_{1}}^{\perp} \sigma_{1}^{\perp} P_{1}(\mu),
\end{aligned}
$$

for $1^{1} P_{1}$ states, where $C_{F}=\left(N_{c}^{2}-1\right) /\left(2 N_{c}\right), C_{G}=N_{c}$ and $L \equiv \alpha_{s}(Q) / \alpha_{s}(\mu)$. 


\section{The definitions of LCDAs of physical $f_{1}$ and $h_{1}$ mesons}

For $f_{1}(1285), f_{1}(1420), h_{1}(1170)$ and $h_{1}(1380)$ mesons, denoted by $B$ in the following, we define the chiral-even LCDAs to be (with $Q \equiv u, d$, or $s$ )

$$
\begin{aligned}
\left\langle B(P, \lambda)\left|\bar{Q}(y) \gamma_{\mu} \gamma_{5} Q(x)\right| 0\right\rangle= & i f_{B}^{D} m_{B} \int_{0}^{1} d u e^{i(u p y+\bar{u} p x)}\left\{p_{\mu} \frac{\epsilon^{*(\lambda)} z}{p z} \Phi_{\|}^{B^{Q}}(u)+\epsilon_{\perp \mu}^{*(\lambda)} g_{\perp}^{(a) B^{Q}}(u)\right. \\
& \left.-\frac{1}{2} z_{\mu} \frac{\epsilon^{*(\lambda)} z}{(p z)^{2}} m_{B}^{2} g_{3}^{B^{Q}}(u)\right\}, \\
\left\langle B(P, \lambda)\left|\bar{Q}(y) \gamma_{\mu} Q(x)\right| 0\right\rangle= & -i f_{B}^{D} m_{B} \epsilon_{\mu \nu \rho \sigma} \epsilon_{(\lambda)}^{* \nu} p^{\rho} z^{\sigma} \int_{0}^{1} d u e^{i(u p y+\bar{u} p x)} \frac{g_{\perp}^{(v) B^{Q}}(u)}{4},
\end{aligned}
$$

and the chiral-odd LCDAs to be

$$
\begin{aligned}
\left\langle B(P, \lambda)\left|\bar{Q}(y) \sigma_{\mu \nu} \gamma_{5} Q(x)\right| 0\right\rangle= & f_{B}^{\perp, D} \int_{0}^{1} d u e^{i(u p y+\bar{u} p x)}\left\{\left(\epsilon_{\perp \mu}^{*(\lambda)} p_{\nu}-\epsilon_{\perp \nu}^{*(\lambda)} p_{\mu}\right) \Phi_{\perp}^{B^{Q}}(u)\right. \\
& +\frac{m_{B}^{2} \epsilon^{*(\lambda)} z}{(p z)^{2}}\left(p_{\mu} z_{\nu}-p_{\nu} z_{\mu}\right) h_{\|}^{(t) B^{Q}}(u) \\
& \left.+\frac{1}{2}\left(\epsilon_{\perp \mu}^{*(\lambda)} z_{\nu}-\epsilon_{\perp \nu}^{*(\lambda)} z_{\mu}\right) \frac{m_{B}^{2}}{p \cdot z} h_{3}^{B^{Q}}(u)\right\} \\
\left\langle B(P, \lambda)\left|\bar{Q}(y) \gamma_{5} Q(x)\right| 0\right\rangle= & f_{B}^{\perp, D} m_{B}^{2}\left(\epsilon^{*(\lambda)} z\right) \int_{0}^{1} d u e^{i(u p y+\bar{u} p x)} \frac{h_{\|}^{(p) B^{Q}}(u)}{2},
\end{aligned}
$$

where the distribution amplitudes are subject to the choices of the normalization constants $f_{B}^{\perp, D}$ with (i) $D \equiv u$ for $f_{1}(1285), h_{1}(1170)$, and (ii) $D \equiv s$ for $f_{1}(1420), h_{1}(1380)$. Here we use the conventions for the decay constants that $f_{f_{1}(1285)}^{\perp, u}=f_{f_{1}(1285)}^{u}, f_{f_{1}(1420)}^{\perp, s}=f_{f_{1}(1285)}^{s}$, $f_{h_{1}(1170)}^{u}=f_{h_{1}(1170)}^{\perp, u}(1 \mathrm{GeV})$, and $f_{h_{1}(1380)}^{s}=f_{h_{1}(1380)}^{\perp, s}(1 \mathrm{GeV})$. The relevant decay constants can be found in Eqs. (91) - (94) and (105)-(108).

\section{References}

[1] V.M. Braun and I.E. Filyanov, Z. Phys. C 48 (1990) 239.

[2] V. M. Braun, G. P. Korchemsky and D. Muller, Prog. Part. Nucl. Phys. 51, 311 (2003) arXiv:hep-ph/0306057.

[3] V. S. Fadin, E. A. Kuraev and L. N. Lipatov, Phys. Lett. B 60, 50 (1975).

[4] E. A. Kuraev, L. N. Lipatov and V. S. Fadin, Sov. Phys. JETP 44, 443 (1976) [Zh. Eksp. Teor. Fiz. 71, 840 (1976)]. 
[5] E. A. Kuraev, L. N. Lipatov and V. S. Fadin, Sov. Phys. JETP 45, 199 (1977) [Zh. Eksp. Teor. Fiz. 72, 377 (1977)].

[6] I. I. Balitsky and L. N. Lipatov, Sov. J. Nucl. Phys. 28, 822 (1978) [Yad. Fiz. 28, 1597 (1978)].

[7] V. L. Chernyak and A. R. Zhitnitsky, Phys. Rept. 112, 173 (1984).

[8] P. Ball, JHEP 9901, 010 (1999) arXiv:hep-ph/9812375.

[9] P. Ball, V. M. Braun, Y. Koike and K. Tanaka, Nucl. Phys. B 529, 323 (1998) arXiv:hep-ph/9802299.

[10] P. Ball and V. M. Braun, Nucl. Phys. B 543, 201 (1999) arXiv:hep-ph/9810475.

[11] H. Yang et al., Phys. Rev. Lett. 94, 111802 (2005) arXiv:hep-ex/0412039].

[12] K. Abe et al. [Belle Collaboration], arXiv:hep-ex/0507096.

[13] B. Aubert et al. [BABAR Collaboration], Phys. Rev. Lett. 97, 051802 (2006) arXiv:hep-ex/0603050].

[14] B. Aubert [BABAR Collaboration], arXiv:hep-ex/0612050.

[15] M. Gronau and J. Zupan, Phys. Rev. D 73, 057502 (2006) arXiv:hep-ph/0512148.

[16] K. C. Yang, Phys. Rev. D72, 034009 (2005) [arXiv:hep-ph/0506040].

[17] K.C. Yang, in preparation.

[18] K. C. Yang, JHEP 0510, 108 (2005) [arXiv:hep-ph/0509337].

[19] J. Govaerts, L.J. Reinders, F. De Viron, and J. Weyers, Nucl. Phys. B 283, 706 (1987).

[20] P. Ball, V. M. Braun and A. Lenz, JHEP 0605, 004 (2006) arXiv:hep-ph/0603063.

[21] K.C. Yang, in preparation.

[22] V. M. Braun, S. E. Derkachov, G. P. Korchemsky and A. N. Manashov, Nucl. Phys. B 553, 355 (1999) arXiv:hep-ph/9902375].

[23] V. M. Braun, E. Gardi and S. Gottwald, Nucl. Phys. B 685, 171 (2004) arXiv:hep-ph/0401158.

[24] M.A. Shifman, A.I. Vainshtein and V.I. Zakharov, Nucl. Phys. B147 (1979) 385.

[25] Here we adopt the convention of conformal generators as given in "Comformal Field Theory", Philippe Di Francesco, Pierre Mathieu, and David Sénéchal, 1997, SpingerVerlag New York, Inc. 
[26] T. Ohrndorf, Nucl. Phys. B 198, 26 (1982).

[27] D. J. Gross and F. Wilczek, Phys. Rev. D 9 (1974) 980;

M. A. Shifman and M. I. Vysotsky, Nucl. Phys. B 186 (1981) 475.

[28] W. M. Yao et al. [Particle Data Group], J. Phys. G 33 (2006) 1.

[29] M. Suzuki, Phys. Rev. D 47 (1993) 1252.

[30] L. Burakovsky and T. Goldman, Phys. Rev. D 57, 2879 (1998) arXiv:hep-ph/9703271.

[31] "An Introduction to Quarks and Partons", F.E. Close, 1979, Academic Press Inc. (London) Ltd.

[32] F. E. Close and A. Kirk, Z. Phys. C 76, 469 (1997) arXiv:hep-ph/9706543; D. M. Li, B. Ma, Y. X. Li, Q. K. Yao and H. Yu, Eur. Phys. J. C 37, 323 (2004) arXiv:hep-ph/0408214]; J. Vijande, F. Fernandez and A. Valcarce, J. Phys. G 31, 481 (2005) arXiv:hep-ph/0411299.

[33] P. Ball and M. Boglione, Phys. Rev. D 68, 094006 (2003) arXiv:hep-ph/0307337].

[34] I. I. Balitsky, D. Diakonov and A. V. Yung, Yad. Fiz. 35 (1982) 1300.

[35] V. M. Braun and A. V. Kolesnichenko, Phys. Lett. B 175, 485 (1986) [Sov. J. Nucl. Phys. 44, 489.1986 YAFIA,44,756 (1986 YAFIA,44,756-773.1986)].

[36] V. M. Belyaev and Y. I. Kogan, Yad. Fiz. 40, 1035 (1984).

[37] I. I. Balitsky, A. V. Kolesnichenko and A. V. Yung, Yad. Fiz. 41 (1985) 282;

[38] P. Ball, V. M. Braun and N. Kivel, Nucl. Phys. B 649, 263 (2003) arXiv:hep-ph/0207307.

[39] P. Ball and R. Zwicky, Phys. Rev. D 71, 014029 (2005) arXiv:hep-ph/0412079.

[40] P. Ball and R. Zwicky, Phys. Lett. B 633, 289 (2006) arXiv:hep-ph/0510338.

[41] I. I. Balitsky and V. M. Braun, Nucl. Phys. B 311, 541 (1989).

[42] P. Ball and R. Zwicky, JHEP 0602, 034 (2006) arXiv:hep-ph/0601086.

[43] Y. J. Kwon and J. P. Lee, Phys. Rev. D 71, 014009 (2005) arXiv:hep-ph/0409133.

[44] J. P. Lee, Phys. Rev. D 69, 114007 (2004) arXiv:hep-ph/0403034].

[45] A. S. Safir, Eur. Phys. J. directC 3 (2001) 15 arXiv:hep-ph/0109232. 
[46] K. C. Yang, W. Y. P. Hwang, E. M. Henley, and L. S. Kisslinger, Phys. Rev. D 47, 3001, (1993); W. Y. P. Hwang and K. C. Yang, ibid, 49, 460 (1994).

[47] Y. Koike and K. Tanaka, Phys. Rev. D 51, 6125 (1995) arXiv:hep-ph/9412310].

[48] Y. Koike and N. Nishiyama, Phys. Rev. D 55, 3068 (1997) arXiv:hep-ph/9609207].

[49] Y. Koike, N. Nishiyama and K. Tanaka, Phys. Lett. B 437, 153 (1998) arXiv:hep-ph/9805460]. 